\title{
Interactive Multiple Objective \\ Asset Allocation
}

by

Songzhi Lin

A project report

presented to Ryerson University

in partial fulfillment of the

requirement for the degree of

Master of Engineering

in the Program of

Mechanical Engineering

Toronto, Ontario, Canada, 2003

@Songzhi Lin 2003 


\section{UMI Number: EC53447}

\section{INFORMATION TO USERS}

The quality of this reproduction is dependent upon the quality of the copy submitted. Broken or indistinct print, colored or poor quality illustrations and photographs, print bleed-through, substandard margins, and improper alignment can adversely affect reproduction.

In the unlikely event that the author did not send a complete manuscript and there are missing pages, these will be noted. Also, if unauthorized copyright material had to be removed, a note will indicate the deletion.

\section{UMI}

UMI Microform EC53447

Copyright 2009 by ProQuest LLC

All rights reserved. This microform edition is protected against unauthorized copying under Title 17, United States Code.

ProQuest LLC

789 East Eisenhower Parkway

P.O. Box 1346

Ann Arbor, MI 48106-1346 


\section{Author's Declaration}

I hereby declare that I am the sole author of this project report.

I authorize Ryerson University to lend this project report to other institutions or individuals for the purpose of scholarly research.

Signature:

I further authorize Ryerson University to reproduce this project report by photocopying or by other means, in total or in part, at the request of other institutions or individuals for the purpose of scholarly research.

Signature: 


\section{Borrower's Page}

Ryerson University requires the signatures of all persons using or photocopying this project report. Please sign below, and give address and date. 


\section{Abstract}

Portfolio efficiency and suitability are two important goals in the asset allocation process involving a financial advisor and clients. In this project report, a multiple objective asset allocation method which is intended to obtain a suitable portfolio for a specific individual investor is proposed. The Analytic Hierarchy Process (AHP) technique is employed as a framework to address an investor's muitiple investment objectives. Single-objective portfolio optimization techniques are integrated into the decision hierarchy. To determine a single-objective optimal portfolio, a method is proposed to measure an investor's risk tolerance. An interactive procedure based on the proposed asset allocation method is presented to implement the allocation process. Comparisons with a single-objective optimization method using a hypothetical example show that improved portfolio efficiency in terms of all objectives can be achieved. Questionnaires, GAMS (General Algebraic Modeling System) programs and spreadsheet models are developed to facilitate the communications between the financial advisor and investor. 


\section{Acknowledgements}

I would like to express my special thank to my academic advisor, Professor Liping Fang, for his guidance, support and numerous hours spent in discussions and reviewing this project report. His help and contributions are essential to the completion of this work. 


\section{Dedication}

To my parents

To my brothers, sister and their families 


\section{Table of Contents}

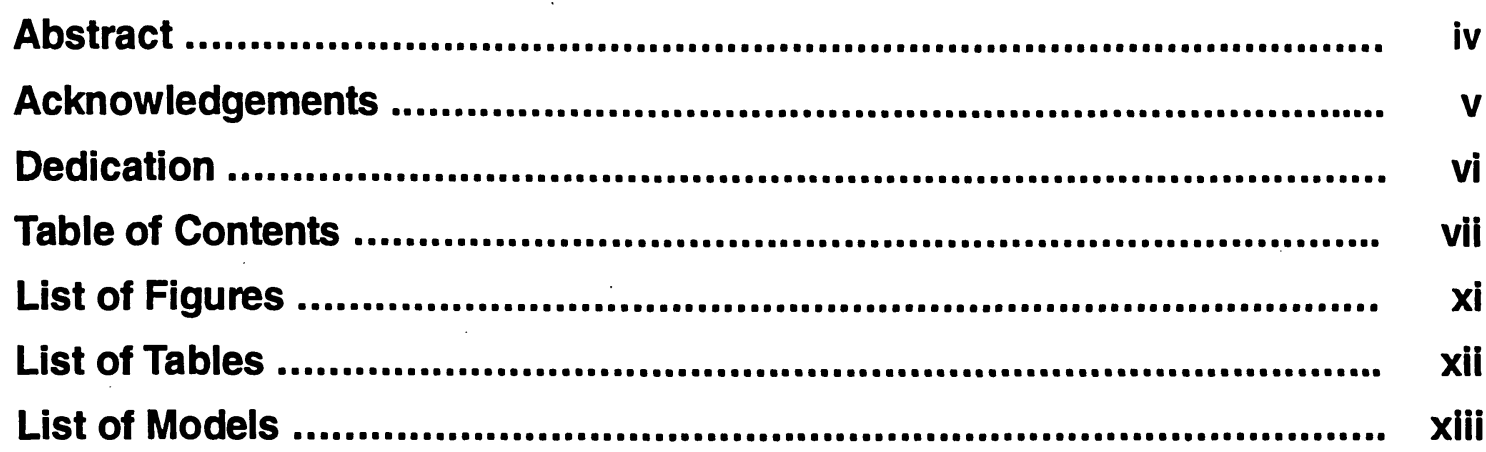

Chapter 1: Introduction ................................................................. 1

1.1 Asset Allocation Process ......................................................... 2

1.1.1 Investment Goals ...................................................... 2

1.1.2 Asset Classes ............................................................. 3

1.1.3 Diversification .......................................................... 4

1.1.4 Portfolio Determination ..................................................... 4

1.2 Asset Allocation's Efficiency and Suitability ................................... 5

1.3 Organization of this Report ....................................................... 6

Chapter 2: Portfolio Theory and Asset Allocation .................................. 8

2.1 Mean-variance Portfolio Theory .............................................. 8

2.2 Alternative to Mean-variance Portfolio Theory .................................. 11

2.3 Efficient Asset Allocation .................................................. 12

2.4 Limitations of Single-objective Optimization Methods .......................... 15

Chapter 3: Multiple-objective Asset Allocation ................................... 17

3.1 The Analytic Hierarchy Process .............................................. 17

3.2 Proposed Multiple-objective Asset Allocation Model ........................... 18

3.3 An Investor's Objectives ...................................................... 20

3.4 Asset Classes and Objectives for an Hypothetical Investor ...................... 23

3.5 The Decision Hierarchy for the Hypothetical Investor .......................... 26

3.6 Assets' Expected Returns, Standard Deviations and Correlations ................. 26

3.7 Pairwise Comparisons of Objectives ......................................... 29

3.8 Consistency Test ........................................................... 33

3.9 Spreadsheet Model for Objective Weighting ................................. 35

Chapter 4: Estimating an Investor's Risk Tolerance ............................. 39

4.1 Traditional Risk Tolerance Measurements ....................................... 39

4.2 Theoretic Foundation for the Proposed Method ................................ 40

4.3 Selection of Reference Portfolios ............................................... 43

4.4 Select the Conservative Reference Portfolio ................................... 44 
4.4.1 Heuristic Method

4.4.2 The Global Minimum Variance Portfolio ................................. 45

4.4.3 Generating the List $\mathrm{A}_{\mathrm{c}}$ of the Candidate Portfolios ........................ 48

4.4.4 Select the Reference Portfolio A Using Questionnaires ...................... 49

4.5 Select the Aggressive Reference Portfolio ...................................... 50

4.5.1 Heuristic method .......................................................... 51

4.5.2 The Maximum Return Portfolio .......................................... 52

4.5.3 Generating the List of $\mathrm{C}_{\mathrm{a}}$ of the Candidate Portfolios ...................... 54

4.5.4 Select the Reference Portfolio C Using Questionnaires .................... 56

4.6 Relative Degree of Preference between Reference Portfolios ................... 57

4.7 Spreadsheet Model for Calculating the Degree of Preference ................... 62

4.8 Construction of Local Optimal Portfolios ..................................... 64

Chapter 5: Complete Portfolios and Comparisons ................................ 66

5.1 The Process of Constructing a Complete Portfolio ............................. 66

5.2 Complete Portfolios for the Hypothetical Investor .............................. 68

5.3 Measurement of Portfolio Efficiency ........................................... 71

5.4 Comparison with Single-objective Optimization ............................. 72

Chapter 6: Conclusions and Future Work .......................................... 75

6.1 Summary of Contributions ................................................... 75

6.2 Future Work ................................................................ 76

Appendices

Appendix 1: GAMS Programs Associated with the Total Nominal Return Objective .................................................................

Appendix 1.1: GAMS Program for Finding the Global Minimum Variance Portfolio in terms of the Total Nominal Return Objective

Appendix 1.2: GAMS Program for Generating the List of Candidate Portfolios in terms of the Total Nominal Return Objective

Appendix 1.3: GAMS Program for Finding the Maximum Return Portfolio in terms of the Total Nominal Return Objective

Appendix 1.4: GAMS Program for Obtaining Local Optimal Portfolios in terms of the Total Nominal Return Objective

Appendix 1.5: GAMS Program for Finding Performance of the Complete Portfolio in terms of the Total Nominal Return Objective

Appendix 1.6: GAMS Program for Obtaining the Optimal Portfolio for a Given Target Return in terms of the Total Nominal Return Objective

Appendix 2: GAMS Programs Associated with the Capital Appreciation

Objective

Appendix 2.1: GAMS Program for Finding the Global Minimum Variance Portfolio in terms of the Capital Appreciation Objective

Appendix 2.2: GAMS Program for Generating the List of Candidate Portfolios in terms of the Capital Appreciation Objective 
Appendix 2.3: GAMS Program for Finding the Maximum Return Portfolio

in terms of the Capital Appreciation Objective

Appendix 2.4: GAMS Program for Obtaining Local Optimal Portfolios

in terms of the Capital Appreciation Objective

Appendix 2.5: GAMS Program for Finding Performance of the Complete portfolio

in terms of the Capital Appreciation Objective

Appendix 2.6: GAMS Program for Obtaining the Optimal Portfolio for a Given

Target Return in terms of the Capital Appreciation Objective

101

Appendix 3: GAMS Programs Associated with the Current Income Objective

Appendix 3.1: GAMS Program for Finding the Global Minimum Variance Portfolio in terms of the Current Income Objective

104

Appendix 3.2: GAMS Program for Generating the List of Candidate Portfolios

in terms of the Current Income Objective

106

Appendix 3.3: GAMS Program for Finding the Maximum Return Portfolio

in terms of the Current Income Objective

108

Appendix 3.4: GAMS Program for Obtaining Local Optimal Portfolios

in terms of the Current Income Objective

Appendix 3.5: GAMS Program for Finding Performance of the Complete Portfolio

in terms of the Current Income Objective

Appendix 3.6: GAMS Program for Obtaining the Optimal Portfolio for a Given

Target Return in terms of the Current Income Objective

114

Appendix 4: GAMS Programs Associated with the After-tax Return Objective .......

Appendix 4.1: GAMS Program for Finding the Global Minimum Variance Portfolio

in terms of the After-tax Return Objective

Appendix 4.2: GAMS Program for Generating the List of Candidate Portfolios

in terms of the After-tax Return Objective

Appendix 4.3: GAMS Program for Finding the Maximum Return Portfolio

in terms of the After-tax Return Objective

Appendix 4.4: GAMS Program for Obtaining Local Optimal Portfolios

in terms of the After-tax Return Objective

Appendix 4.5: GAMS Program for Finding Performance of the Complete Portfolio in terms of the After-tax Return Objective

Appendix 4.6: GAMS Program for Obtaining the Optimal Portfolio for a Given

Target Return in terms of the After-tax Return Objective

Appendix 5: GAMS Programs Associated with the Preservation of Purchasing

Power Objective

Appendix 5.1: GAMS Program for Finding the Global Minimum Variance Portfolio in terms of the Preservation of Purchasing Power Objective

Appendix 5.2: GAMS Program for Generating the List of Candidate Portfolios in terms of the Preservation of Purchasing Power Objective

Appendix 5.3: GAMS Program for Finding the Maximum Return Portfolio in terms of the Preservation of Purchasing Power Objective

Appendix 5.4: GAMS Program for Obtaining Local Optimal Portfolios in terms of the Preservation of Purchasing Power Objective 
Appendix 5.5: GAMS Program for Finding Performance of the Complete portfolio in terms of the Preservation of Purchasing Power Objective

Appendix 5.6: GAMS Program for Obtaining the Optimal Portfolio for a Given

Target Return in terms of the Preservation of Purchasing Power

Objective 140

Appendix 6: GAMS Programs Associated with the Liquidity Objective

Appendix 6.1: GAMS Program for Finding the Global Minimum Variance Portfolio in terms of the Preservation of Purchasing Power Objective

Appendix 6.2: GAMS Program for Generating the List of Candidate Portfolios in terms of the Liquidity Objective

Appendix 6.3: GAMS Program for Finding the Maximum Return Portfolio in terms of the Liquidity Objective

Appendix 6.4: GAMS Program for Obtaining Local Optimal Portfolios

in terms of the Liquidity Objective

Appendix 6.5: GAMS Program for Finding Performance of the Complete Portfolio in terms of the Liquidity Objective

Appendix 6.6: GAMS Program for Obtaining the Optimal Portfolio for a Given

Target Return in terms of the Liquidity Objective

References 


\section{List of Figures}

Figure 2.1: The opportunity set and efficient frontier .................................. 10

Figure 2.2: Determination of optimal complete portfolio with a risk-free asset ......... 13

Figure 2.3: Determination of optimal complete portfolio without a risk-free asset ..... 13

Figure 3.1: Typical AHP hierarchy .................................................. 18

Figure 3.2: General model for multiple-objective asset allocation ..................... 19

Figure 3.3: Investor's objectives and risk profile questionnaire ........................ 22

Figure 3.4: Decision hierarchy for a hypothetical investor ............................. 27

Figure 3.5: Investor's preference for investment objective questionnaire ................ 31

Figure 3.6: Sample Excel model for objective weighting ................................ 36

Figure 3.7: Excel model inputs and formulae for Figure $3.6 \ldots \ldots \ldots \ldots \ldots \ldots \ldots \ldots \ldots . . . \ldots 36$

Figure 4.1: Efficient frontier and indifferent curves .................................. 41

Figure 4.2: Search for the global minimum variance portfolio :......................... 45

Figure 4.3: Flowchart for finding the global minimum variance portfolio ................ 46

Figure 4.4: Generating the list of the conservative reference portfolios .................. 49

Figure 4.5: Example questionnaire for selecting the conservative reference portfolio ... 51

Figure 4.6: Search for the maximum return portfolio .................................. 52

Figure 4.7: Flowchart for finding the maximum return portfolio ......................... 54

Figure 4.8: Generating the list of the aggressive reference portfolios .................. 55

Figure 4.9: Example questionnaire for selecting the aggressive reference portfolio ...... 56

Figure 4.10: Example questionnaire for assessing relative degree of preference

between two reference portfolios ............................................ 58

Figure 4.11: Sample Excel model for calculating an investor's relative degree of preference between two reference portfolios ................................. 62

Figure 4.12: Excel model inputs and formulae for Figure 4.11 ........................ 63 


\section{List of Tables}

Table 3.1: Expected returns and standard deviations of asset classes .................... 29

Table 3.2: Correlations between asset classes ......................................... 29

Table 3.3: Suggested scale for AHP ratio assessment .................................. 30

Table 3.4: Comparison matrix based on the investor's responses ....................... 30

Table 3.5: The average random consistency index ....................................... 34

Table 3.6: Relative importance of six objectives ..................................... 38

Table 4.1: The global minimum variance portfolio for each objective .................. 48

Table 4.2: The maximum return portfolio for each objective ........................... 53

Table 4.3: Reference portfolios for each objective ..................................... 57

Table 4.4: Initial comparison matrices for portfolios A, B, and C ...................... 59

Table 4.5: Comparison matrices for portfolios A, B, and C ............................. 60

Table 4.6: Comparison matrices for the hypothetical investor .......................... 60

Table 4.7: The relative degree of preference for the hypothetical investor ................ 64

Table 5.1: Local optimal and complete portfolios when the investor takes an

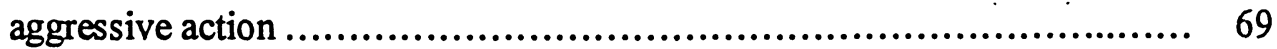

Table 5.2: Local optimal and complete portfolios when the investor takes a

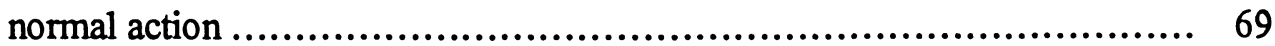

Table 5.3: Local optimal and complete portfolio when the investor takes a conservative

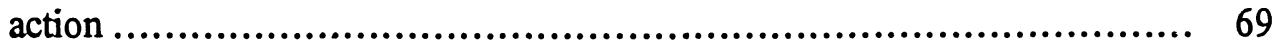

Table 5.4: The performances of a complete portfolio in terms of each objective ........ 70

Table 5.5: Associated efficient portfolios of complete portfolios ....................... 71

Table 5.6: Deviation index and efficiency of complete portfolios ...................... 72

Table 5.7: Performances of the ATR portfolio in terms of each objective ................ 73

Table 5.8: Associated efficient portfolios of the ATR portfolios ......................... 73

Table 5.9: Deviation index and efficiency of the ATR portfolios ........................ 74 


\section{List of Models}

Model 2.1 ............................................................................ 8

Model 2.2............................................................................ 14

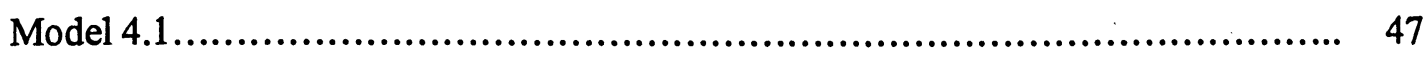

Model 4.2......................................................................... 64 



\section{Chapter 1: Introduction}

Almost every one in the world owns a combination of assets. It can be composed of real assets, such as a house, a car, or furniture, and financial assets, such as cash, stocks, or bonds. Real assets are mostly for personal use in everyday life. There is not much room left to be managed to generate income unless one sells them. Financial assets are resources that can be organized to provide potential future growth and income. Asset allocation is an investment decision process about how to allocate available capital among different types of financial assets. A combination of these assets is called a portfolio. For many individual investors, however, the process of constructing a portfolio can be time-consuming and may be a tough task because of limited knowledge and experience. Financial markets are full of uncertainty. No one can predict what will exactly happen in the future. Investors worry about making wrong decisions. And when investors seek advice from a financial professional, they worry that the advisor may make wrong decisions for them. In order to make a good investment recommendation, the financial advisor should consider multiple dimensions of an investor's objectives and preferences.

Optimization techniques have traditionally been used in asset allocation process. However, an efficient portfolio determined by an optimization model is not necessarily also suitable for a particular investor, partly because the judgment of a portfolio's suitability is largely subjective (Bolster, et al., 1995), and partly because traditional optimization is in practice constructed as a single-objective decision problem. Multiple-objective decision making methods, such as the Analytic Hierarchy Process (AHP) developed by Saaty (1980), are suitable to deal with complex situations where multiple objectives should be considered. However, a suitable portfolio obtained by the AHP normally is not optimal (Bolster. and Warrick, 2000). By integrating the AHP with traditional optimization techniques, it is hoped that a suitable portfolio with improved overall efficiency can be obtained. A major objective of this project report is to present such a multiple objective asset allocation model for individual investors. When using the mean-variance optimization technique to determine a portfolio, an investor's risk tolerance is a key input factor. A theoretically sound and workable measure for an investor's risk tolerance is also presented in this report.

To develop a multiple objective asset allocation model, an understanding of asset allocation 
process is needed. An introduction to this process, as well as portfolio's efficiency and suitability, is given below.

\subsection{Asset Allocation Process}

All decision problems have common elements: criteria of choice, alternatives and the preferred solution(s). The decision alternatives for asset allocation are numerous possible combinations of asset classes. For individual investors, they themselves, or the financial advisor help them, evaluate the alternatives according to their financial goals and considerations, and choose an asset combination that can achieve these goals. This process can be summarized as goal setting, asset class selection, and portfolio determination.

\subsubsection{Investment Goals}

The key for a successful financial advisor is to know what his client needs. And the key for an investor is to know what himself needs. The old saying "knowing the destination is half the journey" indicates the important role of goal setting in asset allocation process. It determines an investor's asset allocation strategy. That is, asset allocation should be consistent with investors' investment goals.

Many investors have a list of concrete goals, such as to buy a house, send children to universities, save enough money to retire early and comfortably, and so on. Some investors would like to defer their income tax to future years or utilize the favorable tax rates on different investment instruments. Some would like to preserve their capital from potential losses. Some are concerned about the purchasing power of their money and would like to seek investments to hedge increased inflation. Many investors seek multiple investment objectives. Most of these goals can be translated into return requirements from investment portfolios.

Investors' financial status, time horizons and stages in their life cycle affect the asset allocation decision. For example, ligh tax-bracket investors may seek investments that generate income from capital gains and dividends, which are taxed at a lower rate than interest income. Different investor risk attitudes also direct the investment portfolio toward more conservative or more aggressive asset combinations. 


\subsubsection{Asset Classes}

If we define asset allocation as spreading investments across a variety of asset classes, a natural question is what asset classes are available for investors. Actually there are thousands of asset alternatives available in the financial markets. In broad terms, cash, bonds, stocks and real estate are four basic asset classes (see Maginn and Tuttle, 1990, p. $10-2$, for the detailed discussions about treating real estate as an asset class in portfolio analysis). They can be subdivided into smaller classes according to certain classification methods. Stocks, for example, can be subdivided into large-cap and small-cap; value and growth; international and domestic; and/or combinations thereof. Bonds can be subdivided into governmental and corporate; short, intermediate, and long term; convertible and non-convertible; coupon and zero-coupon, etc. Each sub-class can be further divided. Each asset class possesses some common properties that can distinguish it from others.

Asset classes provide various investment opportunities as well as different risk and return characteristics. Cash and cash equivalents, such as bank accounts, term deposits, treasury bills, money market mutual funds and other short-term instruments, typically provide interests and generally be considered conservative because of their low risk, high liquidity and abilities to preserve capital. Fixed-income assets, such as corporate bonds, provide potential higher income opportunities but have moderate risk because they are subject to potential default risk for reasons such as bankruptcy. Government bonds rarely have default risk but are still subject to long-term inflation risk and generally have lower interest rates than those of corporate bonds. Historically, stocks have been the best performing asset class among traditional investments (Information Please, 2003). They typically offer greater growth possibilities and good hedge to inflation in long-term. However, their prices fluctuate frequently, sometimes significantly and therefore have high risk of loss. Commercial real estate provides both lease income and potential capital appreciation, but is not frequently traded and has high liquidity risk.

Rational investors prefer higher returns and dislike risk (Markowitz, 1952). However, the natural law of investments is that the higher the returns, the higher are the risks, and vice verse. Although controversial, Efficient Market Hypothesis (Fama, 1995) states that security prices fully reflect all available information of both historical and expected future events, and any efforts to pick up undervalued securities are like "random walk" in markets. 
Asset allocation is neither intended to outperform market nor a tool to "buy low, sell high", but a strategy to protect potential losses and to provide "satisfied" return by combining asset classes into a portfolio.

\subsubsection{Diversification}

Once asset classes are identified, investors should determine how much of each class should be included in the portfolio. That is, how much of funds should be invested in stocks, how much in bonds, and so on. The answer is crucial to long-term portfolio performance. An old adage tells us: "Don't put all the eggs in one basket". If one drops the basket that carries all eggs, one loses all. This saying is well suited to situations of investing. If an investor invests in a number of assets, he prevents himself from large losses linked to one or some poor decisions. Modern portfolio theory (MPT), developed first by Markowitz (1952), goes further than traditional random diversification. Analyses and attentions are not only given to the risks associated to each individual asset (asset class), but also provided to interrelationships among component assets within a portfolio. Diversification works because not all financial assets go up or down at the same time or at the same rate. When stock markets go up, bond markets may go down. While the high-tech sector falls sharply, the food sector may slide only slightly or remain the same. If investors hold assets that do not correlate with each other, the increase in one can offset losses in another. MPT points out that a well-diversified portfolio will achieve higher returns without having to take more risk, or achieve given expected returns for minimal risk. This is the theoretical foundation of asset allocation.

\subsubsection{Portfolio Determination}

In asset allocation process involving a financial advisor and clients, typically the advisor provides available market opportunities (Maginn and Tuttle, 1990, p. 7.12). These opportunities are combinations of several asset classes that are believed to be efficient or suitable based on capital market research results. A final portfolio for a given individual investor is determined by combining the available market opportunities with that investor's preferences, usually the most important one is the investor's risk tolerance (Maginn and 
Tuttle, 1990, p. 7-27). Typically the value of the investor's risk tolerance is evaluated using a designed questionnaire. The responses to the questionnaire are scored. An asset allocation recommendation can be made using rules of thumb based on the accumulated score (Droms and Stauss, 2003), or the score can be transformed into a value of risk tolerance that can be incorporated into an optimization model to determine an optimal asset-mix (Bolster and Warrick, 2000).

\subsection{Asset Allocation's Efficiency and Suitability}

Investors hope to be given a portfolio that is consistent with their financial status as well as investment goals and preferences. But they may be concerned about if the recommended portfolio is the "best" one available. There are two important considerations in asset allocation process: portfolio's suitability and efficiency.

After the birth of Markowitz's mean-variance theory (Markowitz, 1952), traditional research of asset allocation focuses on two major areas: one on the solution techniques of mean-variance optimization, the other on the different definitions of portfolio risks. The studies in the first area lead to the developments of other important components of modern portfolio theory, including index model (Sharpe, 1963), Capital Asset Pricing Model (CAPM) (Sharpe, 1964), and Arbitrage Pricing Theory (APT) (Ross, 1976). The research in the second area leads to various alternative portfolio optimization methods, such as mean absolute deviation optimization (Konno and Yamazaki; 1991). Both areas of research pay attentions to the optimality of a portfolio and distinguish themselves based on what is the most appropriate measure of asset risk and portfolio risk. From a viewpoint of decision making, all of these models generate objective judgments of alternatives. As long as the properties of component assets are known, the output portfolios are deterministic. They are optimal or efficient based on mean-variance sense or mean absolute deviation sense.

The efficient portfolios derived from optimization models are not necessarily also suitable for a particular investor. In many portfolio optimization methods, one of the major investment objectives is often formulated as the objective function that needs to be optimized, while other objectives or considerations come into the optimization models as constraints. A portfolio obtained by this single-objective optimization method is efficient in terms of this objective, but not necessarily also optimal in terms of others. Because of the 
various situations faced by different investors, it may be impossible to find a universally optimal portfolio (Saraoglu and Detzler, 2002). If multiple conflicting objectives coexist and one objective does not significantly dominate others, investors may not think the portfolio is suitable for them. Furthermore, the determinations of portfolio suitability are largely subjective, and financial advisors often have to rely on intuition and past experience to make judgments. Disgruntled investors may sue for damage or file complaints against financial advisors if they believe the recommended investments are not suitable for them (Bolster, et al., 1995).

Professional associations, such as Association for Investment Management and Research (AIMR, 2003), require their members consider the appropriateness and suitability of investment recommendations for clients. However, the research in the suitability of a portfolio appeared in financial literature only in recent years and was relative rare. The Analytic Hierarchy Process (AHP), developed by Saaty (1980), has been used by several researchers to formulate portfolio mix (Khaksari, et al., 1989), to determinate an investor's suitability (Bolster, et al., 1995) and to select mutual funds (Saraoglu and Detzler, 2002). AHP constructs the asset allocation decision problem as a hierarchy. Each objective in the hierarchy is prioritized and its relative importance is weighed. The performance of each asset is evaluated in terms of each objective or sub-objective. A portfolio can be formulated by combining the weighed asset classes. The purpose of using AHP in asset allocation is to ensure that investors' objectives and preferences are reasonably satisfied. Generally, however, the portfolio obtained is not optimal (Bolster and Warrick, 2000).

\subsection{Organization of this Report}

This project report presents an asset allocation method which is intended to obtain a suilable portfolio for a specific individual investor. Improved overall portfolio efficiency can also be achieved. In this proposed method, the AHP is employed as a framework to address an investor's multiple investment objectives. For each objective, an optimization model is used to determine a local optimal portfolio. Interactive communications between the financial advisor and the investor ensure that the asset allocation process effectively reflect the investor's preferences.

This project report is organized as follows. In Chapter 2, the classical mean-variance 
portfolio theory, alternative portfolio optimization methods and efficient asset allocation. techniques are reviewed and their limitations are discussed. In Chapter 3, the Analytic Hierarchy Process (AHP) is first introduced. A multiple-objective asset allocation model which integrates the AHP framework and portfolio optimization techniques is presented. A hypothetical investment problem is constructed to illustrate the application of the proposed model. In Chapter 4, a theoretical formulation of measuring an investor's risk tolerance is derived. An interactive process is designed to ascertain the measurement. In Chapter 5, the process of constructing a complete portfolio is first reviewed. Then the complete portfolios constructed for the hypothetical investment problem are presented. A deviation index is designed to measure the overall efficiency of the complete portfolios and used to compare the results with those obtained using a single-objective optimization method. In Chapter 6, a summary of the author's contributions is provided and some areas of future work are suggested. 


\section{Chapter 2: Portfolio Theory and Asset Allocation}

Modern portfolio theory is the theoretical foundation of efficient asset allocation. It suggests that diversifying a portfolio into different assets increases portfolio return or decreases portfolio risk. Combining efficient market opportunities with an investor's preference, an optimal portfolio can be determined. In this chapter, the classical mean-variance portfolio theory is first introduced and alternative optimization methods are reviewed. The methods of determining optimal portfolios are described. Limitations of single-objective optimization methods are discussed.

\subsection{Mean-variance Portfolio Theory}

The first pioneering contribution to modern portfolio theory.was the seminal work of Dr. Markowitz (1952), who received the 1990 Nobel Prize in Economics (Royal Swedish Academy of Science, 1990). Before Markowitz, the investment management principle was to buy assets thought to be undervalued, and sell them later hopefully for a profit. Markowitz formulated the portfolio selection problem as to take the mean or expected rate of return of a portfolio as the investment return and the standard deviation (or variance) of return of a portfolio as the investment risk. The mechanism of portfolio diversification was quantitatively analyzed. When assets are properly mixed, investors could construct a portfolio that provides a higher overall return without having to undertake more portfolio risk. Markowitz's mean-variance theory has since become the standard framework in modern portfolio management (Michaud, 1998, p. 2).

An efficient portfolio, according to Markotiwz, is defined as one that has the largest expected return for a given level of risk, or the smallest portfolio risk for given level of expected return. The mathematical formulation of this problem is a quadratic programming model (denoted as Model 2.1):

\section{Model 2.1:}

Objective:

Maximize $\quad E\left(R_{P}\right)=\sum_{i=1}^{N} x_{i} E\left(R_{i}\right)$ 
Subject to:

$$
\begin{aligned}
& \sigma_{P}^{2}=\sum_{i=1}^{N} x_{i}^{2} \sigma_{i}^{2}+\sum_{i=1}^{N} \sum_{\substack{j=1 \\
j \neq i}}^{N} x_{i} x_{j} \rho_{i j} \sigma_{i} \sigma_{j} \\
& \sum_{i=1}^{N} x_{i}=1 \\
& x_{i} \geq 0 \quad i=1,2, \ldots, N
\end{aligned}
$$

where: $E\left(R_{P}\right)=$ expected return of portfolio;

$\sigma_{\mathrm{P}}=$ standard deviation of return of portfolio;

$\mathrm{E}\left(\mathrm{R}_{i}\right)=$ expected return for asset class $i$;

$\sigma_{i}=$ standard deviation of return of asset class $i$;

$\rho_{i j}=$ correlation coefficient between returns of asset class $i$ and $j$;

$\mathrm{N}=$ the number of asset classes;

and $\mathrm{x}_{i}=$ proportion of portfolio invested in asset class $i$.

Rational investors would seek efficient portfolios because these portfolios are optimized on both expected returns and risks, which are believed to be the most important considerations to investors. This can be illustrated in Figure 2.1. The circled area in Figure 2.1 is the opportunity set, which is the entire set of all attainable asset combinations. The efficient portfolios are found along the efficient frontier starting from point $\mathrm{K}$ to. Q. The efficient frontier is a concave risk-return trade-off curve in the expected return-standard deviation space that extends from the minimum variance portfolio $\mathrm{K}$ to the maximum return portfolio Q (Elton and Gruber, 1991, p. 53). Each portfolio in the efficient frontier, say, portfolio $G$ is optimal because the expected return of portfolio $G$ is greater than the expected return of all other portfolios within the opportunity set with the same standard deviation, and its standard deviation is less than any other portfolios with the same level of expected return. Portfolio $\mathrm{K}$ is the global minimum variance portfolio because no other minimum-variance portfolio has a smaller risk. Portfolio $Q$ is the maximum return portfolio because no other efficient portfolio has a higher return. 


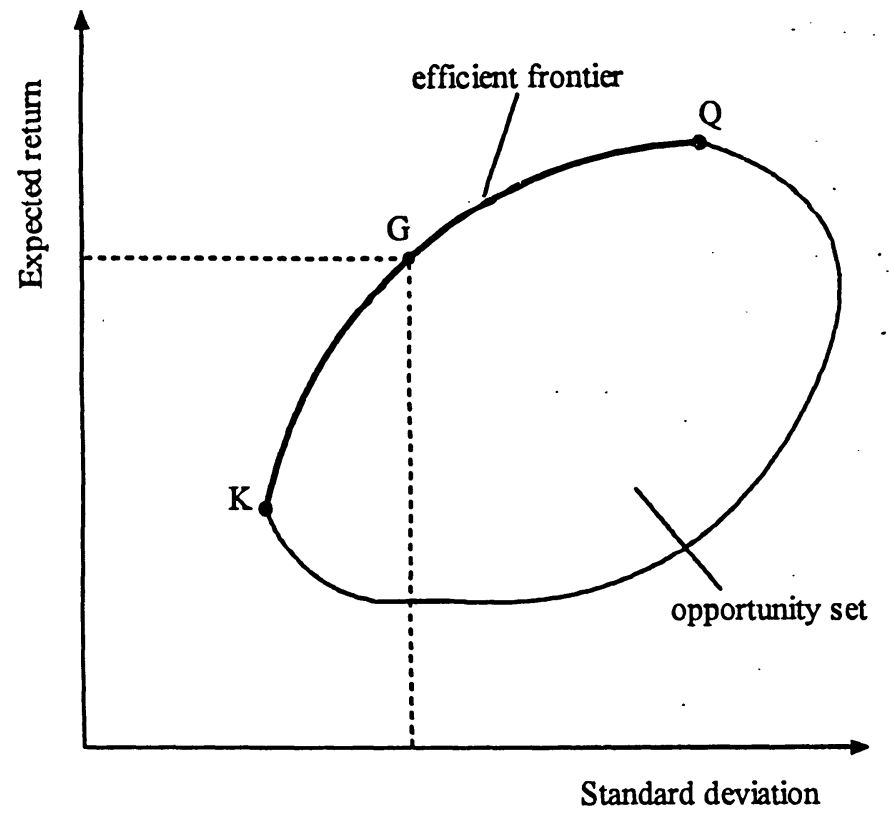

Figure 2.1: The opportunity set and efficient frontier (Source: Binhammer and Sephton, 1998, p. 127)

In Markowitz's mean-variance model, analysis of portfolio risk not only includes the analysis of the risks associated with individual component assets, but also contains the analysis of the co-movements, or correlations, between the component assets within a portfolio. A positive correlation means their returns tend to change in the same direction, while a negative correlation indicates their returns tend to change in the opposite direction. A well-diversified portfolio would eliminate part of the total risk, the nonsystematic risk of a portfolio (Jones, 1998, p. 220). That is, the risk of a portfolio is commonly less than the risk of the any of the component assets, provided that they are not perfectly positively correlated. Thus rational investors diversify to reduce risk.

Assets' expected returns, standard deviations, and the correlation coefficients can be obtained from research results of security analyses. They are inputs in the Markowitz model. In strategic asset allocation, which always employs long-term capital market conditions, these inputs are considered constant (Sharpe, 1987). Weights of assets making up the portfolio are variables that can be manipulated to solve the quadratic programming problem to determine efficient portfolios.

Variant optimization models have been proposed based on Markowitz's work. In particular, 
in 1960s and 1970s, some simplified approximation methods were proposed in order to reduce computational difficulties related to the solution of a quadratic programming model (see Sharpe 1967, 1971; Stone, 1973). Sharpe was the co-recipient of the 1990 Nobel Prize in Economics for having developed the Capital Asset Pricing Model (CAPM) (Royal Swedish Academy of Sciences, 1990). CAPM (Sharpe, 1964) was built on Markowitz portfolio theory. It treated market as a whole as optimal risky portfolio, and gave us the concept of the "beta" -- a coefficient designed to measure the risk of a particular stock relative to the performance of the market portfolio.

\subsection{Alternatives to Mean-variance Portfolio Theory}

Markotiwz's model was built on some assumptions. Two of those assumed that the expected returns of assets are normally distributed and an investor is rational, which means he prefers higher return of the portfolio and smaller standard deviation. Markotiwz's model is known to be valid if these underlying assumptions are satisfied (Konno and Yamazaki, 1991). However, these assumptions may not represent the more sophistical real situations. Some researchers commented that rational investors are not necessarily all risk aversive, and standard deviation (or variance) not necessarily the most appropriate measure of risk (see Cohen, et al., 1987; p. 135). Polsky (1998) stated that even though expected future volatility and correlation are good inputs, there is a limitation to using them as risk measures when taking portfolio options into considerations. Michaud (1998; p. 3) also argued that, the most important limitations of Markowitz's model were its instability and ambiguity. The optimized portfolio was extremely sensitive to input changes and tended to maximize input errors.

Alternative models have been proposed. They include mean-absolute deviation optimization model (Konno and Yamazaki, 1991); mean semi-absolute deviation model (Speranza, 1996), and mean-semivariance model (Hamza and Janssen, 1998). In addition, researchers have proposed alternative portfolio theories that included more moments such as skewness (see Elton and Gruber, 1997). Shortfall risk, downside variance, value-at-risk, and relätive risk are considered as alternative measures of risk (see Chriss and Fanelli, 1998). Other portfolio selection techniques include Monte Carlo simulation approaches (Maginn and Tuttle, 1990, p. 7-46) and utility function evaluations (Maginn and Tuttle, 1990, p. 7-49). 
Among these alternatives, linear programming methods have received increased attentions. For example, Konno and Yamazaki (1991) demonstrated that a linear programming model using mean absolute deviation risk function has computational advantage, among others, over classical Markowitz's model. However, in practice, using linear programming to construct a precise portfolio structure may not be easy. It needs to formulate a variety of linear equality and inequality constraints to reflect the relationships between assets and to control portfolio risk. "From a theoretical point of view, only a MV (mean-variance) optimization framework can optimally use active forecast information" (see Michaud, 1998, p. 32).

\subsection{Efficient Asset Allocation}

The process of portfolio construction in practice is separated into asset allocation and security selection (Bodie, et al, 1997, p. 259). Although the Markowitz model was initially developed for security selection, it has found successful applications in implementing asset allocation strategies. Both of efficient asset allocation and security selection require the formulation of efficient frontier and the determination of an optimal portfolio along the frontier. Security selection generally involves the allocation of hundreds, even thousands, of securities, while asset allocation involves normally 2-20 asset classes. However, studies show that more than $80 \%$ of long-term portfolio performance come from choosing the right combination of assets, not from choosing the right assets (RBC Investments, 2003).

All the portfolios in the efficient frontier are optimal in the mean-variance context. To determine the optimal portfolio for a specific investor, the investor's preferences and the relevant constraints should be combined with the market opportunities. If a risk-free asset is available to an investor, then the determination of the optimal complete portfolio is illustrated in Figure 2.2. The optimal capital allocation line (CAL) is a capital allocation line starting from $R_{f}$ (the return of the risk-free asset) in vertical axis and tangent with the curve of optimal opportunity set (efficient frontier). It has the highest reward-to-variability ratio (Bodie, et al., 1997, p. 246). The point of tangency between the efficient frontier and optimal CAL is the optimal risky portfolio, that is, $P$ in Figure 2.2. An optimal risky portfolio is composed of risky assets only. A complete portfolio is obtained by allocating capitals between the risk-free asset and optimal risky portfolio. The point of tangency between the investor's indifference curve and the optimal CAL is the optimal complete 
portfolio, that is, $\mathrm{T}$ in Figure 2.2. It provides highest utility to the given investor.

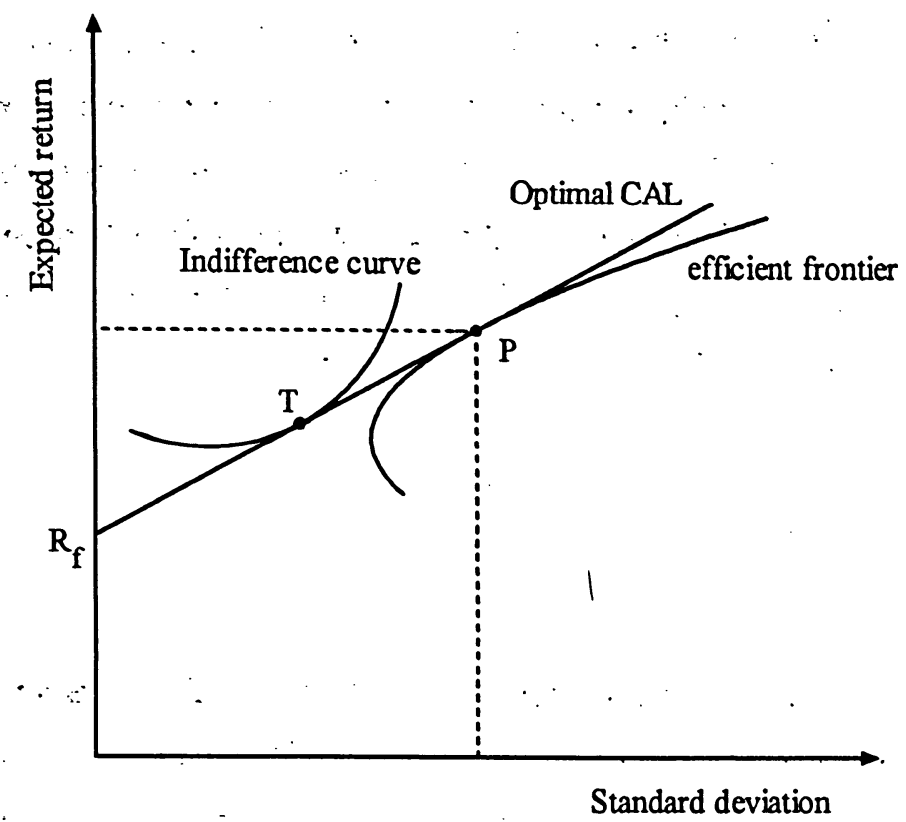

Figure 2.2: Determination of optimal complete portfolio with a risk-free asset (Source: Bodie, et al, 1997, p. 249)

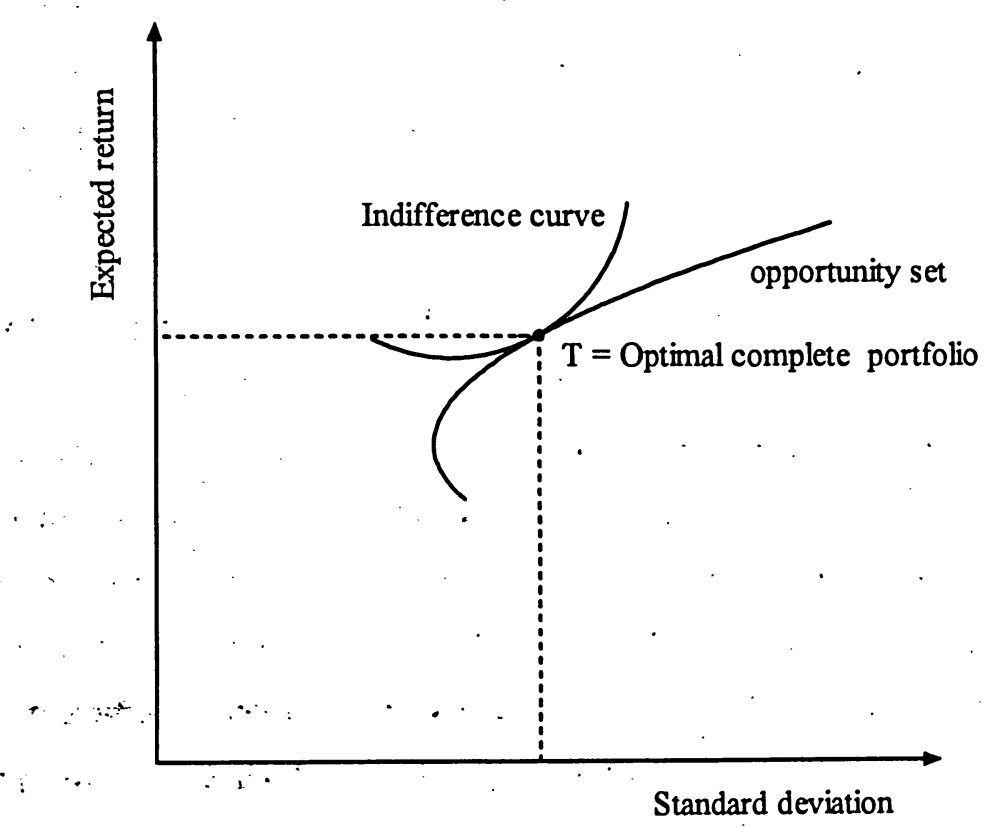

Figure 2.3: Determination of optimal complete portfolio without a risk-free asset (Source: Bodie, et al, 1997, p. 260) 
Actually, the risk-free asset does not exist in real world. The treasury-bills, commonly to be viewed as the risk-free asset, are subject to interest rate risk. If a risk-free asset is not available, as the data used for hypothetical investment example in this project report, the asset allocation decision is to find the optimal risky portfolio that provides the highest utility to the given investor. This is illustrated in Figure 2.3, where the optimal complete portfolio, $T$, is the point of tangency between the investor's indifference curve and the efficient frontier.

An investor's utility function is usually unknown. However, if we assume that an investor's utility function can be represented by a particular type of smooth curve, and the investor has constant risk tolerance, then a reasonable utility function can be derived (Sharpe, 1987). Here an investor's risk tolerance is defined as the added variance for per unit of added expected return, providing the same utility for the investor (Maginn and Tuttle, 1990, p. 7-9). In this utility function, a risk aversive investor considers the risk as a penalty to the utility value. The greater the volatility of a portfolio, or the smaller the risk tolerance, the greater is the penalty. A general formulation of this optimization problem is a quadratic programming model (denoted as Model 2.2). Model 2.2 is showed as follows:

\section{Model 2.2:}

Objective:

$$
\text { Maximize } U=E\left(R_{P}\right)-\frac{\sigma_{P}^{2}}{t}
$$

Subject to:

$$
\begin{aligned}
& \sigma_{P}^{2}=\sum_{i=1}^{N} x_{i}^{2} \sigma_{i}^{2}+\sum_{i=1}^{N} \sum_{\substack{j=1 \\
j \neq i}}^{N} x_{i} x_{j} \rho_{i j} \sigma_{i} \sigma_{j} \\
& E\left(R_{P}\right)=\sum_{i=1}^{N} x_{i} E\left(R_{i}\right) \\
& \sum_{i=1}^{N} x_{i}=1 \quad \\
& x_{i} \geq 0 \quad i=1, \ldots, N
\end{aligned}
$$

where: $U=$ utility of the portfolio provided to the investor; 
$\therefore \therefore t=$ the investor's risk tolerance;

$\mathrm{E}\left(\mathrm{R}_{\mathrm{P}}\right)=$ expected return of portfolio;

$\sigma_{\mathrm{P}}=$ standard deviation of return of portfolio;

$\mathrm{E}\left(\mathrm{R}_{i}\right)=$ expected return for asset class $i$;

$\sigma_{i}=$ standard deviation of return of asset class $i$;

$\rho_{i j}=$ correlation coefficient between asset class $i$ and $j$;

$\mathrm{N}=$ the number of asset classes;

and $\mathrm{x}_{i}=$ proportion of portfolio invested in asset class $i$.

Optimization models using quadratic programming techniques were widely used for implementing efficient asset allocation. Unlike most other approaches, one advantage of these models is that they can effectively reflect and utilize the fact that risk depends' on interactions among assets (Maginn and Tuttle, 1990, p. 7-54).

\subsection{Limitations of Single-objective Optimization Methods}

In asset allocation optimization models, such as Model 2.1 and Model 2.2, an investor's objectives are generally defined in terms of return requirements or a utility function. Constraints are limitations such as budget constraints and nonnegative weights of assets (short sales not allowed). An investor's preferences or other considerations, such as taxation and liquidity, are generally integrated into the objective function or constraints. For example, if after-tax return is of interest, the expected returns, standard deviation and correlations will adopt after-tax values. If an investor imposes specific requirements for some assets, additional linear inequality and equality constraints may be needed to reflect the requirements.

An important message from these analyses is that, traditional portfolio optimization is in practice constructed as a single-objective decision problem. This modeling method may not be consistent with some real world characteristics of an investor's investment goal. For example, an investor, who seeks to maximize the expected total return while seeks to obtain current income, may find the model's objective is to maximize the total return, while the income objective comes as one of constraints. This model actually treats these two objectives as follows: the total return objective can only be achieved within such a framework that current income objective must be fully met. It doesn't consider the relative 
importance between income objective and total return objective. It is intuitive that if the investor feels the total return is much more important, he may be willing to give up certain current income in order to achieve larger total return if these two goals are actually conflicting with each other. Or, if the investor believes the current income is more important, he would be eager to relax some total return requirements. There can be a trade-off between two objectives.

Another limitation of using single-objective portfolio optimization is that if multiple objectives do exist, they cannot be optimized at a time. Under the criteria of different objectives, the same portfolio may perform differently, sometimes significantly. For example, the total return consists of two components, income (interest or dividends) and capital gain (loss). A portfolio whose majority of assets are stocks may perform better, in terms of capital gain objective, than a portfolio whose majority of assets are bords in the long term. However, the later portfolio generally performs better than former in terms of current income objective. Another example, real estate assets may perform better than bonds in terms of real return. However, because of its illiquidity, an investor who is very concerned with asset liquidity may avoid to invest in it. The result is the fraction of the real estate is reduced in the complete portfolio despite its superiority in terms of real return objective. In these situations, the single-objective optimization solution actually may not be optimal in terms of the investor's overall objectives. 


\section{Chapter 3: Multi-objective Asset Allocation}

Because of limitations of traditional portfolio modeling methods, it is desirable to consider an asset allocation problem as a multiple-objective decision problem. In this chapter, a multiple objective asset allocation method is proposed. This approach is intended to construct a portfolio that is consistent with multiple objective investment needs of an individual investor, while at the same time aim to improve the portfolio's overall efficiency in terms of multiple objectives. Trade-offs between different objectives are considered. The Analytic Hierarchy Process (AHP), initially developed by Saaty (1980), is employed to develop a multiple-objective decision hierarchy. Single-objective portfolio optimization techniques-are integrated into the model to construct local optimal portfolios. The basic procedure of this method is: (1) to identify the investor's objectives; (2) to evaluate the relative importance of these objectives; (3) for each objective, to construct a local optimal portfolio using an optimization model; and (4) to obtain the complete portfolio by combining the local optimal portfolios according to their weights.

\subsection{The Analytic Hierarchy Process}

The Analytic Hierarchy Process (AHP) provides a proven, effective means to structure a complex multi-attribute decision problem. It begins by discomposing an unstructured multiple attribute decision problem into components. In its simplest form, this structure consists of three levels: goal, criteria and alternative levels. A typical AHP hierarchy is shown in Figure 3.1. Each component can be further decomposed into subcomponents at appropriate level of details. The process continues to the lowest level of hierarchy. After the decision hierarchy is constructed, relative priorities of one component over other components are evaluated and represented by numerical weights. Using the AHP, decision makers don't need to make an absolute judgment or assessment over all elements. Instead, decision makers are only required to make a relative assessment between two elements at a time. This makes decision process easier. For example, if there are six investment objectives, and an investor wants to evaluate their importance, clearly it is difficult to evaluate them directly. However, it is easier to compare one objective to another. In the final step of AHP, alternatives are ranked according to their weighted performance in terms of each criterion. 
Level 1

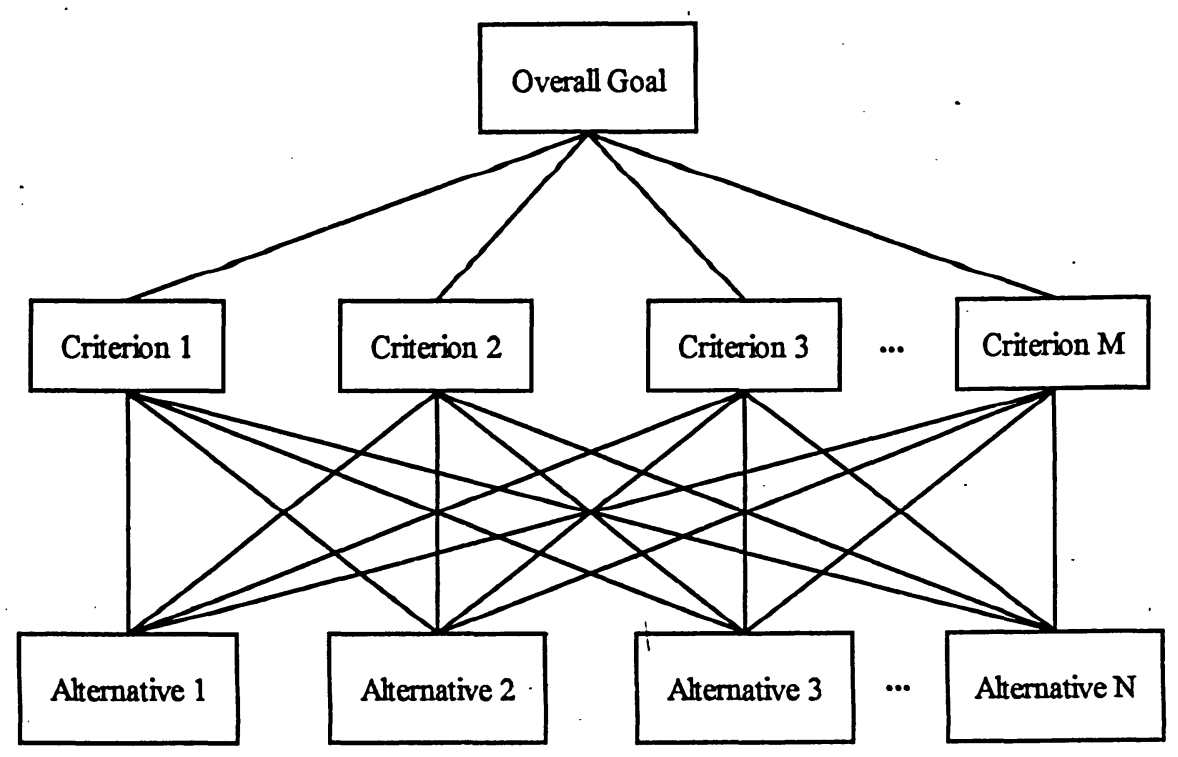

Figure 3.1: Typital AHP hierarchy

The AHP has been applied to many disciplines, such as conflict resolution and group decision making (Saaty, 1982), supplier selection (Muralidharan, et al., 2002); formulation of portfolio mix (Khaksari, et al., 1989) and determination of an investor's suitability (Bolster, et al., 1995). The AHP approach in asset allocation incorporates multiple dimensions of an investor's preferences into decision hierarchy, and considers the priorities of different objectives. Therefore, although the suitability of a portfolio recommendation is largely subjective, it is believed that the asset allocation generated by the AHP is consistent with the investor's objectives and preferences (Saraoglu and Detzler, 2002).

\subsection{Proposed Multiple-objective Asset Allocation Model}

A "suitable" portfolio developed by traditional AHP is normally not optimal in a mean-variance sense. "There is no guarantee that the resulting portfolio will be the highest return available at the given level of expected risk" (Bolster and Warrick, 2000). In order to improve its efficiency, an asset allocation model which integrates the AHP decision hierarchy with traditional optimization techniques is proposed. The general hierarchy structure for $\mathrm{M}$ objectives and $\mathrm{N}$ asset classes is illustrated in Figure 3.2. In this hierarchy, the overall goal, located at the top, is the desired complete portfolio. The second level 
consists of M local optimal portfolios, each of which is associated with the investor's one objective. The weights of local optimal portfolios are the same as those of associated objectives, which are evaluated using AHP priority analysis method. Each local portfolio can be obtained by solving an appropriate single-objective portfolio optimization model, which is shown in the third level of this hierarchy. The inputs for each optimization model are parameters needed to be estimated for the associated model. For mean-variance optimization, the parameters can be expected returns, standard deviations, correlations of asset classes and the investor's risk tolerance: The bottom level consists of $\mathrm{N}$ asset classes that will be weighted and used to make up the local optimal portfolios. The overall goal is achieved by combining weighted local optimal portfolios. That is, the complete portfolio is obtained by combining the local optimal portfolios which are weighted according to their relative.importance.

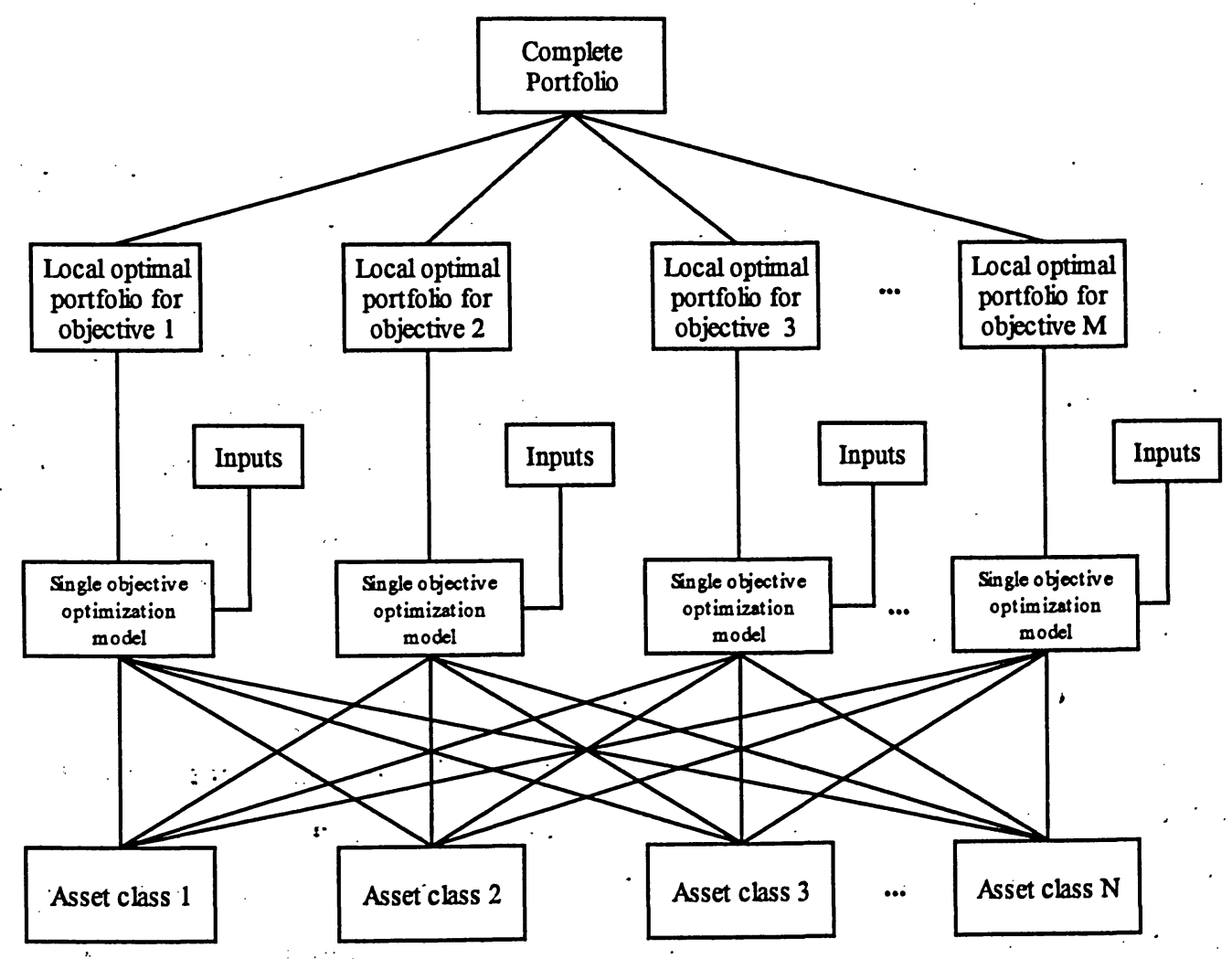

Figure 3.2: General model for multiple-objective asset allocation 
The proposed model can be explained as follows:

Let $W_{i j}$ denote the local weight of asset class $i$ in local portfolio $j$, where $i \in\{1,2, \ldots, \mathrm{N}\}$ and $\mathrm{N}$ is the number of asset classes; $j \in\{1,2 ; \ldots, \mathrm{M}\}$ and $\mathrm{M}$ is the number of objectives (also the number of local portfolios). Let $W_{j}$ denote the weight of local portfolio $j$ in the complete portfolio. Then the sum of local weights of all asset classes in local portfolio $j$ is equal to 1 :

$$
\sum_{i=1}^{N} W_{i j}=1 \quad j \in\{1,2, \ldots, M\}
$$

and the sum of the weights of local portfolios in the complete portfolio is equal to 1:

$$
\sum_{j=1}^{M} W_{j}=1
$$

For each individual asset class $i$, its weight in the complete portfolio, $x_{i}$, is equal to the sum of weighted local weights of asset class $i$ in M numbers of local portfolios:

$$
x_{i}=\sum_{j=1}^{M} W_{j} W_{i j} \quad i \in\{1,2, \ldots, N\}
$$

It can be derived from formulae (3.2.1), (3.2.2) and (3.2.3) that, the sum of the weights of each asset class in the complete portfolio is equal to 1 :

$$
\sum_{i=1}^{N} x_{i}=\sum_{j=1}^{N} \sum_{i=1}^{M} W_{j} W_{i j}=1
$$

\subsection{An Investor's Objectives}

After the decision hierarchy is constructed, the information about an investor's objectives should be obtained. Objectives vary from person to person and may change from time to time according to the investor's financial status and preferences. Some investors may seek assets that would grow in value and/or provide income, and want returns as large as possible. For others, the real future asset value, i.e., value measured in terms of purchasing power, may be more relevant. For yet others, they may be more concerned with the after-tax return. In research using the AHP to model multi-objective portfolio selection problems, the criteria used for choosing objectives vary significantly (see Bolster and Warrick, 2000; and Saraoglu and Detzler, 2002). Therefore, each investor should be treated individually. 
One way to ascertain an investor's objectives is to use appropriately designed questionnaires. A sample questionnaire, as shown in Figure 3.3, is designed for gathering information about an investor's objectives and general. risk profile. 'Note that this questionnaire is not a replacement for obtaining the details of an investor's financial status, such as income sources, savings, and the structure of financial assets, which can be processed separately.

This questionnaire serves for three purposes. First, it serves for compliance with regulatory requirements. For example, AIMR code of ethics and standards of professional conduct requires the Charted Financial Analysts (CFA) should "know" their customers before an investment recommendation is made (AIMR, 2003). Second, this questionnaire can be used to gather the necessary information for determining an investor's investment objectives. Based on this information, a financial advisor can design a customized questionnaire to assess the priority of an investor's objectives.

Third, this questionnaire can be used to give the advisor general profile about an investor's risk tolerance. This information is helpful to the advisor when he selects the starting point for generating a reference portfolio list. Details about this topic will be discussed in Chapter 4. Unlike most of existing questionnaires, however, it does not provide a "measure" of an investor's risk tolerance. The reason is, the numerical values of risk tolerance, as defined in Sharpe's (1987) utility function, do not indicate the aggressiveness of a specific investor. A similar numerical value of risk tolerance, say, 50, may mean very aggressive in one situation while in others may mean relative conservative, depending on the choice of the reference portfolios. 
This questionnaire is designed for gathering an investor's objective requirements and assessing the investor's risk attitude.

1. What interval is your age in?
A. 30 or under
B. $30-44$
C. 44-54
D. 54-64
E. 65 or above

2. When do you expect to begin withdrawing money from your portfolio?
A. Less than 2 years
B. $2-4$ years
C. 5-7 years
D. $8-10$ years
Specification:

3. Inflation is the rise in prices over time. Long-term investors should be aware that, if portfolio returns, or returns of any assets are less than inflation rate, the ability to purchase goods and service might actually decline. Instead, the dollar wealth you receive from your investment are nominal returns, which don't reflect the change of purchasing power of your wealth. Which of these two kinds of wealth is in your concerns, nominal wealth or real wealth?
A. Nominal wealth
B. Real wealth
C. Both

4. Investment returns generally consist of capital gains and regular stream of income, such as dividends or interests. Which of them do you concern about?
A. Capital gains
B. Regular income
C. Both

5. Government considers the increase in funds, or the return on investment, to be taxable. In Canada, capital gains are taxed at a lower rate than dividend incomes, and in turn, dividend incomes are taxed lower than interest incomes. This means different asset mix in your investment will have different impact on your after-tax income. What statement below would you choose?
A. After-tax returns are really important.
B. I don't care about taxation as long as I am making money.

6. Do you think it is important to choose an investment that can provide an opportunity to defer taxation of capital gains and/or interest to future years?
A. Yes,
B. I don't care about this.

Figure 3.3: Investor's objectives and risk pro file questionnaire (to be continued) 
7. Some assets have relative low liquidity compared to others. This means if you hold these assets, and in case of having to sell them in a short time, you have to accept lower prices than market prices. However, these assets may have higher average investment returns and less volatility. Are you financially able to accept a low level of liquidity in your investment portfolio?

A. Yes

B. I do have a liquidity requirement for my investment portfolio.

8. Investing involves a trade-off between risk and return. Generally investors who expect to receive higher long-term average returns should expect to experience greater price fluctuations and higher potential for loss, and vice verse. Considering this, which statement best describes your investment goals?

- A. I prefer investments with little or no fluctuation in value and I am willing to accept lower long-term returns associated with these investments.

B. I prefer investments that can provide moderate levels of returns, and am willing to accept moderate levels of risks.

C. I prefer investments that can provide maximal long-term returns, and I am willing to accept large fluctuations in value:

9. Historically, markets have experienced periods of catastrophic short-term price fall. Suppose you own a well-diversified portfolio that fell by $20 \%$ in one day, how would you react?
A. I would buy more of this portfolio.
B. I would not change my portfolio.
C. I would sell part of my portfolio.
D. I would sell all of my portfolio.

10. Which statement below best describes your experience and knowledge about investing?
A. I am a novice in financial market and don't have much knowledge about investing.
B. I have some experience in stock market but I don't have much knowledge about investing.
C. I have prior experience with stocks and bonds investment, and I have general knowledge of investing.
D. I am a very experienced investor and understand how the stock and bond markets work.

Figure 3.3: Investor's objectives and risk profile questionnaire (continued)

\subsection{Asset Classes and Objectives for a Hypothetical Investor}

An asset class is a group of assets that share some common properties. In broad terms, cash (or cash equivalents), bonds, stocks and real estate are four basic asset classes. The remaining asset classes are composed of precious metals and other assets, which are closely held and traded not. frequently. Each of the asset classes can be subdivided into a number of smaller asset classes according to certain classification methods. For the 
hypothetical investor in this project report, six asset classes are employed for illustrative purpose. They are large-cap stocks (denoted as LaStock), small-cap stocks (denoted as SmStock), long-term corporate bonds (denoted as LCBond), long-term government bonds (denoted as LGBond), Treasury bills (denotes as T-Bill) and real estate (denoted as RealEst). Also, it is assumed that the financial advisor has learned from the investor's responses to the questionnaire shown in Figure 3.3 that the hypothetical investor has six major investment objectives: total nominal return (denoted as TNR), capital appreciation (denoted as CA), current income (denoted as CRI), after-tax return (denoted as ATR), preservation of purchasing power (denoted as PP), and liquidity (denoted as LD). The PP and LD objectives should be transformed into return requirements that can be used as objective functions for associated optimization models. These objectives are described below.

\section{Total Nominal Return (TNR)}

This is the total return that is not adjusted for the inflation factor. In dollar value, total return of investment is the sum of capital gains and income received in the holding period. In this project report, the returns of asset classes or portfolios are defined in terms of percentage. Therefore, total nominal return of investment is defined as the sum of nominal capital appreciation yield and the nominal income.yield in the holding period. An asset's total nominal return can be written as:

$$
\text { TNR }=\text { Capital appreciation }+ \text { Income }
$$

\section{Capital Appreciation (CA)}

Nominal capital appreciation (CA) is the difference between what the.investor paid for an asset purchased and what he may realize when the asset is sold. It can be writteri as:

$$
\mathrm{CA}=(\text { Ending price }- \text { Beginning price }) / \text { Beginning price }
$$

\section{Current Income (CRI)}

For stocks, current income consists of dividends that the investor receives from the shares 
he holds: For T-bills and bonds, current income is the interest income. For real estate, current income is the lease income (assuming the property held is commercial real estate). The formula for nominal current income can be written as:

$$
\mathrm{CRI}=(\text { Income received in the holding period }) / \text { Beginning price }
$$

\section{After-tax Return (ATR)}

Interests, dividends, and capital gains are subject to government taxation. Canadian tax system rewards equity investment for growth (capital appreciation) more than equity investment for dividend income, and in turn, rewards dividend income more than interest income. Here it is assumed that the hypothetical investor is in the top tax bracket (i.e., taxable income above $\$ 104,648$ in Ontario), and the following combined (federal and provincial) marginal rate is used to adjust the total nominal return: interest and ordinary income (46.41\%), capital gains (23.20\%), Canadian dividend (31.34\%) ( $\mathrm{CCH}, 2003$, p. xii). Real estate incomes are treated as ordinary incomes.

\section{Preservation of Purchasing Power (PP)}

Nominal returns capture only the growth rate of an investment; real returns measure the growth rate of purchasing power of the wealth. There are differences between nominal and real returns because the inflation rate varies from period to period. Here a proxy, inflation-adjusted total return, is used to represent the objective of preservation of purchasing power. Adjustments can be made by subtracting the inflation rate from the total nominal returns. In practice, the Consumer Price Index (CPI) is usually used to adjust the numbers (Maginn and Tuttle, 1990, p. 2-8).

\section{Liquidity (LD)}

Liquidity is usually defined as an asset's ability to be sold and converted to cash approximately at current market prices. In order to be sold quickly, some assets may have to be sold in a larger discounted value. Or the large blocks of sell in a short period will result in the fall on the sale price, thus reduce the realized cash value. Cash and money market instruments, such as Treasury bills and commercial papers, where the bid-asked 
spread is small, are the most liquid assets, and real estate is among the least liquid. Here a proxy, liquidity-adjusted return (IAR), is used to represent the strength of an asset in serving with the liquidity objective. The formula for liquidity-adjusted return can be written as:

$$
I A R=T N R \times L I
$$

$L I$ is an asset's liquidity index, which is defined as the relative performance of one asset according to the needed time for this asset to be sold in the full market price. The needed liquidity time is transformed linearly to a scale of 0 to 1 , with the best value (the shortest time) 1 and the worse value (the longest time) 0 , using the following formula:

$$
L I_{i}=\frac{W-T_{i}}{W-B}
$$

where $W$ denotes the longest time, $B$ denotes the shortest time, and $T_{i}$ denotes the needed time for asset $i$ to be sold in the full market value. In the hypothetical example, it is assumed that the needed time for large-cap stocks, small-cap stocks, long-term corporate bonds, long-term government bonds, T-bills and real estate are 3, 14, 7, 7, 2 and 50 days, respectively.

\subsection{The Decision Hierarchy for the Hypothetical Investor}

Following Markowitz (1952), it is assumed that, for each of the six objectives, standard deviation (or variance) is the appropriate measure for asset risk and portfolio risk. Then mean-variance optimization is the appropriate method to obtain each local optimal portfolio. The complete decision hierarchy for the hypothetical investor is shown in Figure 3.4.

\subsection{Assets' Expected Returns, Standard Deviations and Correlations}

As stated in Chapter 2, the mean-variance optimization method utilizes active market estimation data. In the proposed model, Model 2.2 in Section 2.3 is employed to construct a local optimal portfolio for each objective. Model 2.2 requires estimates of two types of 
inputs: one consists of the expected return and risk of each asset, and the correlations between each asset's return with that of others; the other one is the investor's risk tolerance. The first type of parameters, discussed below, can be obtained from market research, which are identical for each investor regardless of his risk tolerance. The investor's risk tolerance should be evaluated according to the investor's personal preference. The details about the measurement of an investor's risk tolerance will be discussed separately in Chapter 4 .

Since asset allocation deals with future return expectations of portfolios, expected values should be used. Expected returns are distinguished from historical returns. Historical returns record the performances of an asset over a specified historical period. They are known with certainty. Expected returns measure the estimates of returns over some future period. They are probabilistic in nature.

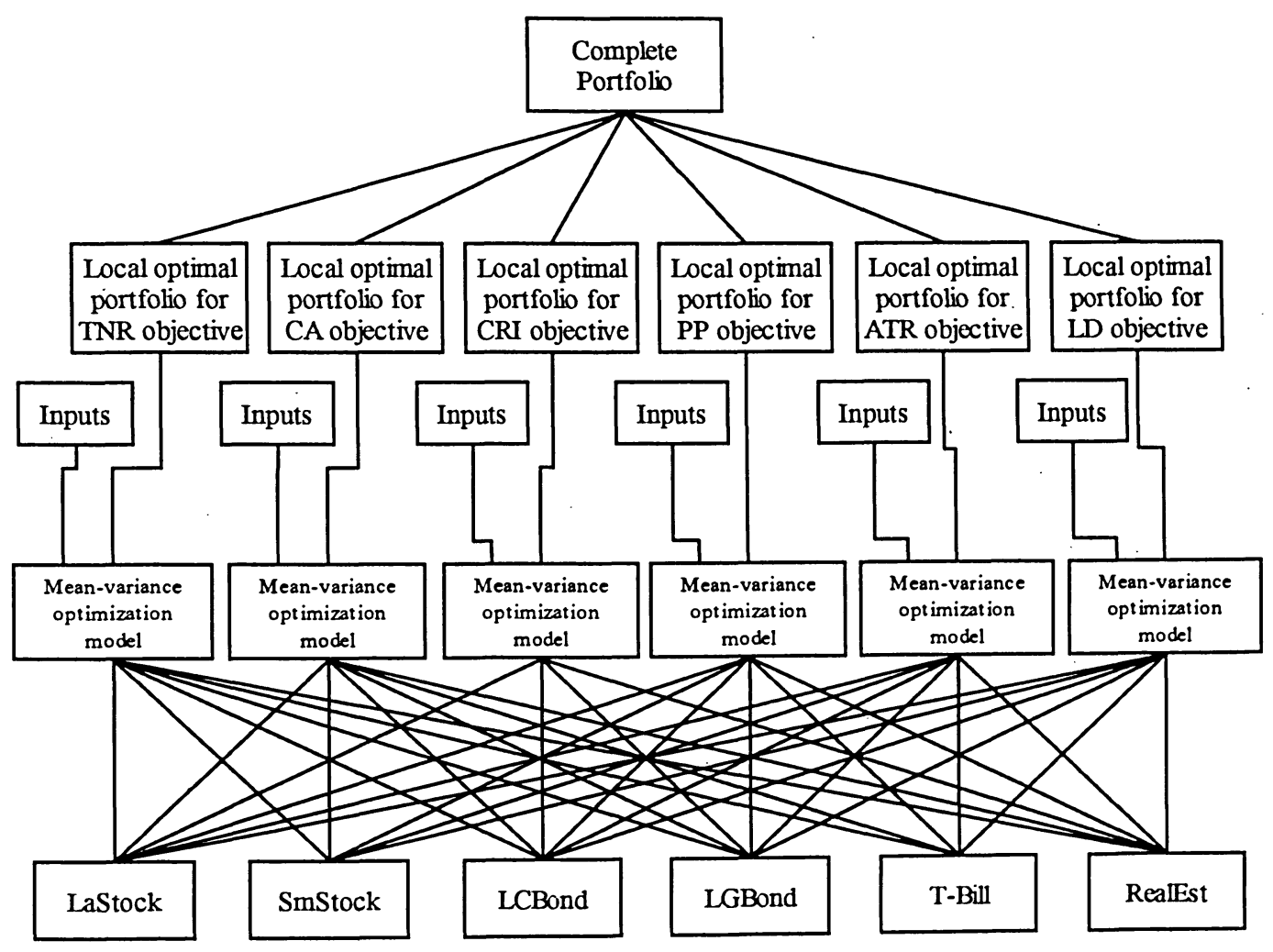

Figure 3.4: Decision hierarchy for a hypothetical investor 
Expected return of an asset is often estimated by using scenario analysis. It is the weighted average of returns in all possible scenarios, where the weights are the occurrence probability of each return. Thus, the expected retum of an asset can be written as (Bodie, et al., 1997, p. 181):

$$
E(R)=\sum_{i=1}^{l} p_{i} R_{i}
$$

Where $E(R)$ is the asset's expected return; $R_{i}$ is the estimated return for $i$ th possible scenario, and $p_{i}$ is the associated probability of $i$ th scenario. $l$ is the number of possible scenarios.

Under Markowitz's mean-variance theory, an asset's risk is measured by the variance (or standard deviation) of expected return. For scenario analysis, the variance is calculated by the weighted squared deviations of the expected return in all possible scenarios. Symbolically, it can be written as (Bodie, et al., 1997, p. 181):

$$
\sigma^{2}=\sum_{i=1}^{l} p_{i}\left[R_{i}-E(R)\right]^{2}
$$

For this project report, because of the practical limitations, historical data are adopted in the illustrative example, which are shown in Table 3.1 and Table 3.2. Researcl shows that historical data are quite useful for estimating standard deviations, reasonably useful for predicting correlations, and virtually useless for estimating expected returns (Maginn and Tuttle, 1990, P. 7-37). Moreover, the time horizons of the data used are not consistent with each other. From these points of view, the data adopted in this project serve only for illustrative purpose.

In Table 3.1, returns and standard deviations of real estate are partially adopted from Ciochetti et al. (2003) for the period 1978-2002. For each of the other five assets, the total nominal return (TNR), capital appreciation (CA), current income (CRI), and inflation-adjusted return (PP) are partially adopted from Ibboston Associates for period 1926-1988 (Maginn and Tuttle, 1990, p. 2-9). The data for after-tax return (ATR) and liquidity-adjusted return (LD) are adjusted data using methods discussed in Section 3.4. 
Table 3.1: Expected returns (return) and standard deviations (STD) of asset classes (\%)

\begin{tabular}{cccccccccccccc}
\hline & \multicolumn{2}{c}{ TNR } & \multicolumn{2}{c}{ CA } & \multicolumn{2}{c}{ CRI } & \multicolumn{2}{c}{ PP } & \multicolumn{3}{c}{ ATR } & \multicolumn{2}{c}{ LD } \\
\hline & return & STD & return & STD & return & STD & return & STD & return & STD & return & STD \\
\hline LaStock & 12.1 & 20.9 & 7.3 & 17.6 & 4.8 & 3.3 & 8.8 & 21.1 & 8.9 & 15.4 & 11.9 & 20.5 \\
SmStock & 17.8 & 35.6 & 12.8 & 27.8 & 5.0 & 8.0 & 14.3 & 34.9 & 13.3 & 26.5 & 13.4 & 26.7 \\
LCBond & 5.3 & 8.4 & -0.6 & 4.9 & 5.9 & 3.5 & 2.4 & $10: 0$ & 3.2 & 5.0 & 4.8 & 7.5 \\
LGBond & 4.7 & 7.5 & -0.4 & 4.6 & 4.9 & 2.9 & 1.7 & 8.5 & 2.6 & 4.2 & 4.2 & 6.7 \\
T-Bill & 3.6 & 3.3 & -0.1 & 1.0 & 3.7 & 2.4 & 0.5 & 4.4 & 2.0 & 1.8 & 3.6 & 3.3 \\
RealEst &. .7 .5 & 15 & 2.3 & 11.7 & 5.2 & 3.3 & 4.3 & 15 & 4.6 & 8.0 & 0.0 & 0.0 \\
\hline
\end{tabular}

The correlation data, shown in Table 3.2, are adopted from Ibboston Associates (Maginn and Tuttle, 1990, p. 2-21, p. 2-37). Correlations between real estate and other assets are data for : the period of 1947-1984. Correlations between large stocks, small stocks, long-term.corporate bonds; long-term government bonds; and Treasury bills are data for the period of 1926-1988. It is assumed in this project report that these correlation data are good estimates for returns of all six objectives under study.

Table 3.2: Correlations between asset classes

\begin{tabular}{ccccccc}
\hline & LaStock & SmStock & LCBond & LGBond & TBill & RealEst \\
\hline LaStock & 1.00 & 0.82 & 0.19 & 0.11 & -0.07 & -0.06 \\
SmStock & 0.82 & 1.00 & 0.08 & -0.01 & -0.08 & 0.06 \\
LCBond & 0.19 & 0.08 & 1.00 & 0.93 & 0.19 & -0.08 \\
LGBond & 0.11 & -0.01 & 0.93 & 1.00 & 0.22 & -0.09 \\
T-Bill & -0.07 & -0.08 & 0.19 & 0.22 & 1.00 & 0.38 \\
RealEst & -0.06 & 0.06 & -0.08 & -0.09 & 0.38 & 1.00 \\
\hline & & - & & & &
\end{tabular}

\subsection{Pairwise Comparisons of Objectives}

In AHP, decision makers express their preferences for a set of alternatives by constructing a matrix of pairwise comparisons in terms of one criterion. This matrix $D_{m}$, shown below, is a positive reciprocal matrix satisfying the requirements of $d_{i j}>0$ and $1 / d_{j i}=d_{i j}$, where $i, j$ $=1,2, \ldots, m$, and $m$ is the number of alternatives. Each element $d_{i j}$ in the matrix represents the decision maker's subjective judgment of alternative $i$ over $j$. 


$$
D_{m}=\left[\begin{array}{cccc}
d_{11} & d_{12} & \cdots & d_{1 m} \\
d_{21} & d_{22} & \cdots & d_{2 m} \\
\vdots & \vdots & \ddots & \vdots \\
d_{m 1} & d_{m 2} & \cdots & d_{m m}
\end{array}\right]
$$

Pairwise comparison judgments are made using a 1-9 scale. The suggested scale by Saaty (1980) is shown in Table 3.3. For example, if an element $i$ is judged to be moderately important than element $j$, a " 5 " number is entered as the value for the pairwise comparison judgment $d_{i j}$ in the matrix $D_{m}$, while its reciprocal value, " $1 / 5$ ", is entered for the pairwise comparison judgment $d_{j i}$.

Table 3.3: Suggested scale for AHP ratio assessments

\begin{tabular}{ll}
\hline The ratio for attribute.I over attribute II should be: \\
\hline 1 & If the two attributes are judged to be equally important \\
3 & If attribute I is judged to be slightly more important than attribute II \\
5 & If attribute I is judged to be moderately more important than attribute II \\
7 & If attribute I is judged to be strongly more important than attribute II \\
9 & If attribute I is judged to be extremely more important than attribute II \\
$2,4,6,8$ & If intermediate values between two adjacent judgments are needed \\
\hline
\end{tabular}

(Source: Hobbs and Meier, 2000, p. 78)

An investor's relative preference can be evaluated by a designed questionnaire. For the illustrative asset allocation example, a sample questionnaire is shown in Figure 3.5. After the investor makes the responses to the questionnaire, the scale values are entered into the comparison matrix. For the illustrative purpose, it is assumed that the hypothetical investor makes the responses shown in Table 3.4. Since the matrix reflecting the investor's actual attitude may not be known, this comparison matrix in Table 3.4 is the observed matrix.

Table 3.4: Comparison matrix based on the investor's responses

\begin{tabular}{ccccccc}
\hline & TNR & CA & CRI & ATR & PP & LD \\
\hline TNR & 1 & 5 & 3 & $1 / 2$ & 4 & 2 \\
CA & $1 / 5$ & 1 & $1 / 2$ & $1 / 7$ & $i$ & $1 / 3$ \\
CRI & $1 / 3$ & 2 & 1 & $1 / 5$ & 2 & $1 / 2$ \\
ATR & 2 & 7 & 5 & 1 & 6 & 4 \\
PP & $1 / 4$ & 1 & $1 / 2$ & $1 / 6$ & 1 & $1 / 2$ \\
LD & $1 / 2$ & 3 & 2 & $1 / 4$ & 2 & 1 \\
\hline
\end{tabular}


This questionnaire is designed for the purpose of assessing your preference over different investment objectives. Please use the scale explained in the following table to compare the relative importance of each investment objective. The former objective mentioned in each question is referred as objective I, and the latter objective is referred as objective II.

\begin{tabular}{cl} 
& \multicolumn{1}{c}{ Suggested scale for pairwise comparison assessments } \\
\hline Scale & \multicolumn{1}{c}{ Description } \\
\hline 1 & If objective I and objective II are judged to be equally important \\
3 & If objective I is judged to be slightly more important than objective II \\
5 & If objective I is judged to be moderately more important than objective II \\
7 & If objective I is judged to be strongly more important than objective II \\
$2,4,6,8$ & If objective Iis judged to be extremely more important than objective II \\
\hline $1 / 3$ & If intermediate values between two adjacent judgments are needed \\
$1 / 5$ & If objective II is judged to be slightly more important than objective I \\
$1 / 7$ & If objective II is judged to be moderately more important than objective I \\
$1 / 9$ & If objective II is judged to be extremely more important than objective I \\
$1 / 2,1 / 4,1 / 6,1 / 8$. If intermediate values between two adjacent judgments are needed
\end{tabular}

\section{Questions:}

1. Do you think which scale best describes the relative importance between Total Nominal Return and Capital Appreciation?

$$
\begin{array}{llllllllllllllllll}
1 & 1 & 2 & 3 & 4 & 5 & 6 & 7 & 8 & 9 & 1 / 2 & 1 / 3 & 1 / 4 & 1 / 5 & 1 / 6 & 1 / 7 & 1 / 8 & 1 / 9
\end{array}
$$

2. Do you think which scale best describes the relative importance between Total Nominal Return and Current Income?

$\begin{array}{llllllllllllllllll}1 & 2 & 3 & 4 & 5 & 6 & 7 & 8 & 9 & 1 / 2 & 1 / 3 & 1 / 4 & 1 / 5 & 1 / 6 & 1 / 7 & 1 / 8 & 1 / 9\end{array}$.

3. Do you think which scale best describes the relative importance between Total Nominal Return and After-tax Total Return?

$$
\begin{array}{llllllllllllllllll}
1 & 2 & 3 & 4 & 5 & 6 & 7 & 8 & 9 & 1 / 2 & 1 / 3 & 1 / 4 & \cdot 1 / 5 & 1 / 6 & 1 / 7 & 1 / 8 & 1 / 9
\end{array}
$$

4. Do you think which scale best describes the relative importance between Total Nominal Return and Preservation of Purchasing Power?

$\begin{array}{llllllllllllllllll}1.2 & 3 & 4 & 5 & 6 & 7 & 8 & 9 & 1 / 2 & 1 / 3 & 1 / 4 & 1 / 5 & 1 / 6 & 1 / 7 & 1 / 8 & 1 / 9\end{array}$

5. Do you think which scale best describes the relative importance between Total Nominal Return and Liquidity?

$\begin{array}{llllllllllllllllll}1 & 2 & 3 & 4 & 5 & 6 & 7 & 8 & 9 & 1 / 2 & 1 / 3 & 1 / 4 & 1 / 5 & 1 / 6 & 1 / 7 & 1 / 8 & 1 / 9\end{array}$

Figure 3.5: Investor's preferences for investment objective questionnaire (to be continued) 
6. Do you think which scale best describes the relative importance between Capital Appreciation and Current Income?

$\begin{array}{lllllllllllllllll}1 & 2 & 3 & 4 & 5 & 6 & 7 & 8 & 9 & 1 / 2 & 1 / 3 & 1 / 4 & 1 / 5 & 1 / 6 & 1 / 7 & 1 / 8 & 1 / 9\end{array}$

7. Do you think which scale best describes the relative importance between Capital Appreciation and After-tar Total Return?

$\begin{array}{llllllllllllllllll}1 & 2 & 3 & 4 & 5 & 6 & 7 & 8 & 9 & 1 / 2 & 1 / 3 & 1 / 4 & 1 / 5 & 1 / 6 & 1 / 7 & 1 / 8 & 1 / 9\end{array}$

8. Do you think which scale best describes the relative importance between Capital Appreciation and Preservation of Purchasing Power?

$\begin{array}{llllllllllllllllll}1 & 2 & 3 & 4 & 5 & 6 & 7 & 8 & 9 & 1 / 2 & 1 / 3 & 1 / 4 & 1 / 5 & 1 / 6 & 1 / 7 & 1 / 8 & 1 / 9\end{array}$

9. Do you think which scale best describes the relative importance between Capital Appreciation and Liquidity?

$\begin{array}{llllllllllllllllll}1 & 2 & 3 & 4 & 5 & 6 & 7 & 8 & 9 & 1 / 2 & 1 / 3 & 1 / 4 & 1 / 5 & 1 / 6 & 1 / 7 & 1 / 8 & 1 / 9\end{array}$

10. Do you think which scale best describes the relative importance between Current Income and After-tar Total Return?

$\begin{array}{llllllllllllllllll}1 & 2 & 3 & 4 & 5 & 6 & 7 & 8 & 9 & 1 / 2 & 1 / 3 & 1 / 4 & 1 / 5 & 1 / 6 & 1 / 7 & 1 / 8 & 1 / 9\end{array}$

11. Do you think which scale best describes the relative importance between Current Income and Preservation of Purchasing Power?

$\begin{array}{lllllllllllllllll}1 & 2 & 3 & 4 & 5 & 6 & 7 & 8 & 9 & 1 / 2 & 1 / 3 & 1 / 4 & 1 / 5 & 1 / 6 & 1 / 7 & 1 / 8 & 1 / 9\end{array}$

12. Do you think which scale best describes the relative importance between Current Income and Liquidity?

$\begin{array}{lllllllllllllllll}1 & 2 & 3 & 4 & 5 & 6 & 7 & 8 & 9 & 1 / 2 & 1 / 3 & 1 / 4 & 1 / 5 & 1 / 6 & 1 / 7 & 1 / 8 & 1 / 9\end{array}$

13. Do you think which scale best describes the relative importance between After-tax Total Return and Preservation of Purchasing Power?

$\begin{array}{lllllllllllllllll}1 & 2 & 3 & 4 & 5 & 6 & 7 & 8 & 9 & 1 / 2 & 1 / 3 & 1 / 4 & 1 / 5 & 1 / 6 & 1 / 7 & 1 / 8 & 1 / 9\end{array}$

14. Do you think which scale best describes the relative importance between After-tax Total Return. and Liquidity?

$\begin{array}{lllllllllllllllll}1 & 2 & 3 & 4 & 5 & 6 & 7 & 8 & 9 & 1 / 2 & 1 / 3 & 1 / 4 & 1 / 5 & 1 / 6 & 1 / 7 & 1 / 8 & 1 / 9\end{array}$

15. Do you think which scale best describes the relative importance between Preservation of Purchasing Power and Liquidity?

$\begin{array}{lllllllllllllllll}1 & 2 & 3 & 4 & 5 & 6 & 7 & 8 & 9 & 1 / 2 & 1 / 3 & 1 / 4 & 1 / 5 & 1 / 6 & 1 / 7 & 1 / 8 & 1 / 9\end{array}$

Figure 3.5: Investor's preferences for investment objective questionnaire (continued)

The priority vector can be obtained from the given comparison matrix. In mathematical terms, the normalized principle eigenvector of matrix $D_{m}$ is the vector of priorities. For 
matrix $D_{m}$, a good estimate of the principal eigenvector (EV) can be described as to multiply the $m$ elements in each row and take the $m$ th root (Saaty, 1980, p. 19):

$$
E V=\left[\begin{array}{c}
b_{1} \\
b_{2} \\
\vdots \\
b_{m}
\end{array}\right]
$$

:where:

$$
b_{i}=\left(d_{i 1} \times \ddot{d}_{i 2} \times \cdots \times d_{i m}\right)^{1 / m} \quad i \in\{1,2, \ldots, m\}
$$

The normalized principal eigenvector is obtained by dividing each element in the eigenvector by the sum of all its elements. The formula is written as:

$$
\begin{aligned}
& \text { Normalized } \quad E V=\left(b_{1}^{\prime}, b_{2}^{\prime}, \cdots, b_{m}^{\prime}\right)^{T} \\
& \text { where } b_{i}^{\prime}=b_{i} / \sum_{j=1}^{m} b_{j} \quad i \in .\{1,2, \ldots, m\}
\end{aligned}
$$

\subsection{Consistency Test}

Before determining the normalized principal eigenvector is a good estimate of the relative weights of the six investment objectives, the consistency of the investor's responses should be tested. Many times investors may not fully understand their own preferences and often make inconsistent preference responses (Saraoglu and Detzler, 2002). For example, suppose an investor has made expression that capital appreciation (CA) is more important than current income (CRI). The investor also believes the current income objective is more important than the preservation of purchasing power (PP) objective. The same investor may also make a response that preservation of purchasing power is more important than capital appreciation, which is inconsistent with his previous answer. Psychological research also shows that consistency of comparisons declines as the number of elements increases (Khaksari, et al., 1989). That is, the consistency of responses are normally not perfect. A suitable asset allocation plan can be achieved only after the investor makes reasonable consistent responses.

A matrix $D_{m}$ of order $m$ is said to be consistent, as defined by Saaty (1980), if it satisfies the following transitivity requirements: 


$$
d_{i j}=d_{i k} \times d_{k j} \quad \text { for } i, \quad j, \quad k=1,2, \ldots, m .
$$

For example, if $d_{12}=2$, and $d_{23}=3$, in a consistent matrix the value of $d_{13}$ has to be 6 .

One of the important advantages of AHP approach is that it can check the consistency of comparison matrix using its built-in mechanism. Consistency is tested by using consistency index and consistency ratio, a technique suggested by Saaty (1980, 1994). Consistency index $(C I)$ is a measure of the deviation of an observed matrix from a consistent matrix. If we denote $\lambda_{\max }$ the largest eigenvalue of observed matrix of pairwise comparisons, then the consistency index $(C I)$ can be written as:

$$
C I=\frac{\lambda_{\max }-m}{m-1}
$$

The value of $\lambda_{\max }$, formulated as (3.8.3), is considered an estimation of $m$. The closer of the value of $\lambda_{\max }$ to $m$, the more consistent is matrix $D_{m}$.

$$
\lambda_{\max }=\max _{i} \sum_{j=1}^{m} d_{i j} \frac{b_{j}}{b_{i}}
$$

where $b_{i}, b_{j}(i, j=1,2, \ldots, m)$ are the elements of principal eigenvector of matrix $D_{m}$.

The average consistency index of randomly generated reciprocal matrices is called average consistency index $(R I)$. Consistency ratio is the ratio of consistency index $(C I)$ to average consistency index $(R I)$ of the same order matrices, which can be written as:

$$
C R=\frac{C I}{R I}
$$

For 1-10 order of matrix, the values of $R I$ can be taken from Table 3.5 (Saaty, 1994).

Table 3.5: The average random consistency index

\begin{tabular}{ccccccccccc}
\hline Matrix order $m$ & 1 & 2 & 3 & 4 & 5 & 6 & 7 & 8 & 9 & 10 \\
\hline$R I$ & 0.00 & 0.00 & 0.52 & 0.89 & 1.11 & 1.25 & 1.35 & 1.40 & 1.45 & 1.49 \\
\hline
\end{tabular}

Source: Saaty (1994).

Saaty (1994) also suggested that, the consistency of a comparison matrix is acceptable if the required consistency ratio $(C R)$ is less than or equal to 0.1 . If the consistency of an 
observed matrix is accepted, the elements in the normalized principal eigenvector are considered the good estimates of the weights of objectives.

When a pairwise matrix fails the consistency test, and if the number of order is large, it is difficult to revise the matrix without the assistance of computer. A number of algorithms have been developed to improve the consistency of pairwise comparisons. For example, $\mathrm{Xu}$ and Wei (1999) developed a method to modify a given matrix to acceptable consistency without requiring the initial respondent to make any revisions. Peters and Zelewski (2003) developed a heuristic algorithm to adjust an observed inconsistent matrix iteratively to improve the consistency. Ishizaka and Lusti (2003) described an expert module application which can assist the decision maker to build a consistent matrix or a matrix with tolerated error.

\subsection{Spreadsheet Model for Objective Weighting}

Spreadsheets are powerful tools for performing tasks involving related data such as calculating objective weights and performing consistency test. For the hypothetical asset allocation problem, we design a model using Microsoft Excel as shown in Figure 3.6. Input data and formula or functions used in this model are illustrated in Figure 3.7.:

This model functions as follows: users (investors or financial advisors) input the scale values obtained from the questionnaire given in Figure 3.5. The number of independent inputs is equal to the number of questions. For this example, it is 15 . Comparisons on principal diagonal are all 1s. Other values are reciprocal values of corresponding independent inputs and can automatically be filled using Excel built-in formula. Once the data have been input, the principal eigenvector of the comparison.matrix is calculated automatically and shown in cell range H6:H11. The normalized principal eigenvector is shown in cell range I6:I11.

The consistency of the matrix is tested at the same time. The eigenvalues of this matrix are shown in cells J6:J11. They are obtained using the following formula:

$$
\lambda_{i}=\sum_{j=1}^{6} d_{i j} \frac{b_{j}}{b_{i}} \quad i=1,2, \ldots 66 \quad(3.9 .1)
$$

The maximum eigenvalue is shown in cell J14 using Excel function. MAX(J6:J11). The 
value of average consistency index $R I$ (shown in cell E17) is taken from Table 3.5 for the order of matrix $m=6$. The consistency index $C I$ and consistency ratio $C R$ are calculated using formula (3.8.2) and formula (3.8.4), respectively.

\begin{tabular}{|c|c|c|c|c|c|c|c|c|c|c|}
\hline 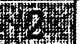 & \multicolumn{10}{|c|}{ This model is designed for calculating the weights of objectives } \\
\hline 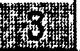 & \multicolumn{10}{|c|}{ and preforming consistency tests } \\
\hline 4 & & & & & $\cdot$ & & & & & \\
\hline 54t) & & TNR & $\mathrm{CA}$ & CRI & ATR & PP & LD & bi & Normal & $\lambda_{i}$ \\
\hline 4 & THR & 1 & 5 & 3 & $1 / 2$ & 4 & 2 & 1.979 & 0.245 & 6.018 \\
\hline & $\mathrm{CA}$ & $1 / 5$ & 1 & $1 / 2$ & $1 \pi$ & 1 & $1 B$ & 0.410 & 0.051 & 6.046 \\
\hline 8 & CRI & $1 / 3$ & 2 & 1 & $1 / 5$ & 2 & $1 / 2$ & 0.715 & 0.088 & 6.091 \\
\hline 1) & ATR & 2 & 7 & 5 & 1 & 61 & 4 & 3.448 & 0.426 & 6.071 \\
\hline 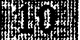 & PP & $1 / 4$ & 1 & $1 / 2$ & $1 / 6$ & 1 & $1 / 2$ & 0.467 & 0.058 & 6.075 \\
\hline 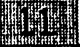 & ID & $1 / 2$ & 3 & 2 & $1 / 4$ & 2 & 1 & 1.070 & 0.132 & 6.090 \\
\hline 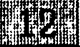 & & & & & & & Sum $=$ & 8.089 & 1.000 & \\
\hline 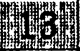 & & & & & & & & . & . & \\
\hline 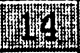 & \multicolumn{6}{|c|}{ Consistency test: (1: pass; 0 : fail) } & 1 & & $\lambda_{\max }=$ & 6.091 \\
\hline 5 & \multicolumn{4}{|c|}{ Order of matrix $m=$} & 6 & & & & & \\
\hline 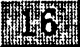 & \multicolumn{4}{|c|}{ Consistency Index $\mathrm{CI}=$} & 0.018 & & & & & \\
\hline 4t & \multicolumn{4}{|c|}{ Random Index $\mathrm{RI}=$} & 1.25 & & & & & \\
\hline & \multicolumn{4}{|c|}{ Consistency Ratio $\mathrm{CR}=$} & 0.015 & & & & & \\
\hline a & & & & & & & & & & \\
\hline
\end{tabular}

Figure 3.6: Sample Excel model for objective weighting

\begin{tabular}{|rl|}
\hline Cell & \multicolumn{1}{c|}{ Values, formulae or functions } \\
\hline B6, C7, D8, E9, F10, G11 & $=1$ \\
$\mathrm{~B} 7, \mathrm{~B} 8, \mathrm{~B} 9, \mathrm{~B} 10, \mathrm{~B} 11$ & $=1 / \mathrm{C} 6,1 / \mathrm{D} 6,1 / \mathrm{E} 6,1 / \mathrm{F} 6,1 / \mathrm{G} 6$, respectively \\
$\mathrm{C} 8, \mathrm{C} 9, \mathrm{C10}, \mathrm{C} 11$ & $=1 / \mathrm{D} 7,1 / \mathrm{E} 7,1 / \mathrm{F} 7,1 / \mathrm{G} 7$, respectively \\
$\mathrm{D} 9, \mathrm{D} 10, \mathrm{D} 11$ & $=1 / \mathrm{E} 8,1 / \mathrm{F} 8,1 / \mathrm{G} 8$, respectively \\
$\mathrm{E} 10, \mathrm{E} 11$ & $=1 / \mathrm{F} 9,1 / \mathrm{G} 9$, respectively \\
$\mathrm{F} 11$ & $=1 / \mathrm{G} 10$ \\
$\mathrm{C} 6: \mathrm{G} 6, \mathrm{D} 7: \mathrm{G} 7, \mathrm{E} 8: \mathrm{G} 8, \mathrm{F9}: \mathrm{G} 9, \mathrm{G} 10$ & (Obtained from the investor's responses) \\
$\mathrm{H} 6$ & $=$ POWER(PRODUCT(B6:G6),1/6) \\
\hline
\end{tabular}

Figure 3.7: Excel model inputs and formulae for Figure 3.6 (to be continued) 


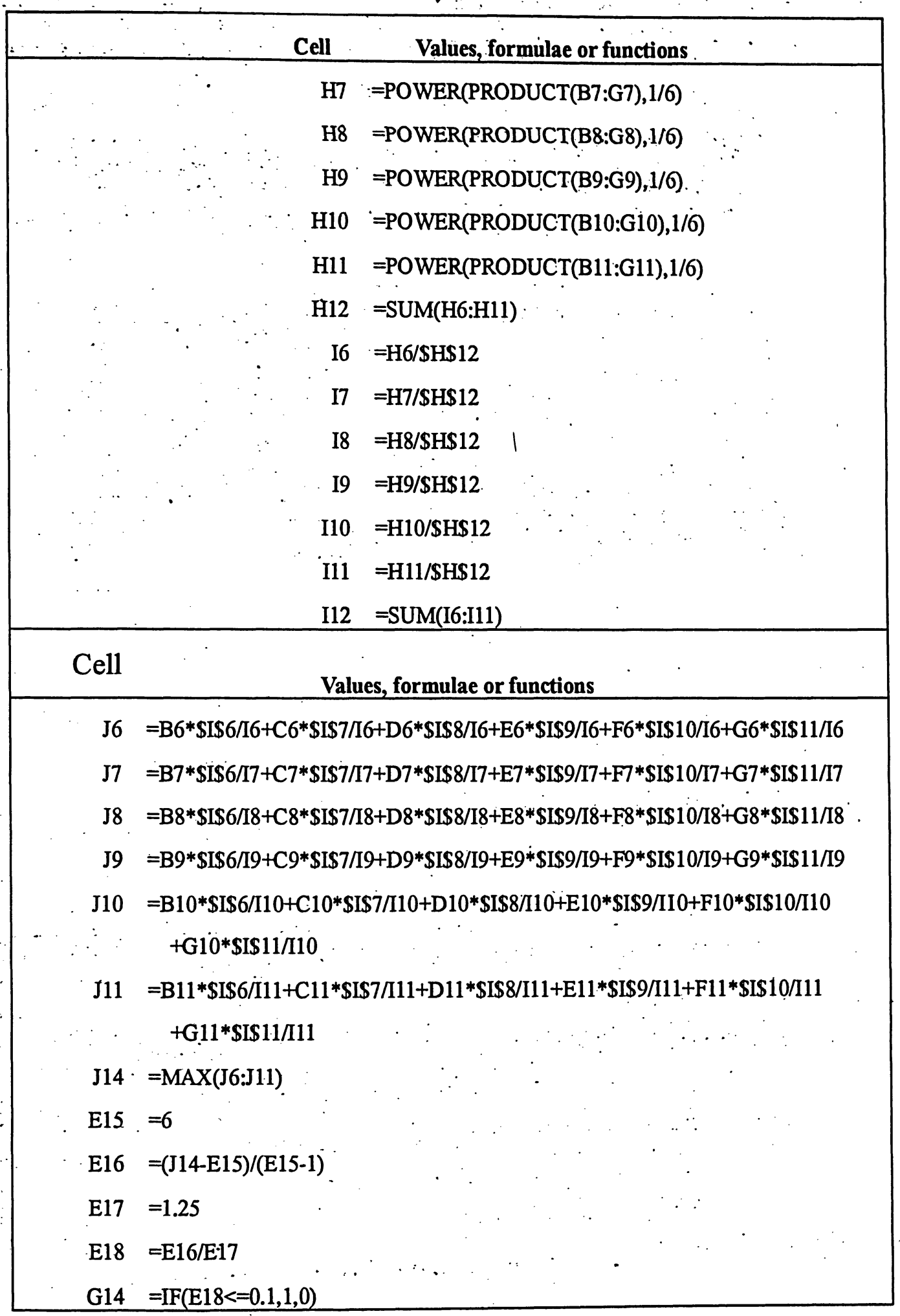

Figure 3.7: Excel model inputs and formulae for Figure 3.6 (continued) 
The consistency test result is shown in cell G14. It is calculated using Excel function $\operatorname{IF}(\mathrm{E} 18<=0.1,1,0)$. It means if the consistency test is passed $(C R \leqslant 0.1)$, the answer is "Yes" (" 1 " in cell G14). If the test is not passed ( $C R>0.1$ ), the answer is "No" ("O" in cell G14), and the investor's responses to the questionnaire should be revised to improve consistency. In this example $C R<0.1$, the consistency of the observed matrix is accepted. Therefore the elements of normalized principal eigenvector (cell range 16:I11 in Figure 3.6) represent the corresponding weights for six objectives. They are shown in Table 3.6.

Table 3.6: Relative importance of six objectives

\begin{tabular}{ccccccc}
\hline & TNR & CA & CRI & ATR & PP & LD \\
\hline Weight $W_{j}$ & 0.245 & 0.051 & 0.088 & 0.426 & 0.058 & 0.132 \\
\hline
\end{tabular}




\section{Chapter 4: Estimating an Investor's Risk Tolerance}

Assets' expected returns, standard deviations and correlations were discussed in Chapter 3. These inputs, however, represent only half of the ingredients required for determining an optimal and suitable portfolio. In this chapter, another important input factor, an investor's risk tolerance, will be discussed, and a method to measure it based on Sharpe's (1987) utility function definition is proposed. In the proposed method, an investor's risk tolerance is defined as the ratio of added portfolio variances to added portfolio returns, considering the investor's relative degree of preference between two reference portfolios. The investor selects his own reference portfolios. The AHP technique is used to evaluate the relative degree of preference between reference portfolios. Risk tolerance obtained from the proposed method can be entered directly as one input into Model 2.2 to determine a local optimal portfolio.

\subsection{Traditional Risk Tolerance Measurements}

Modern portfolio theory indicates that the asset allocation decision process can be separated into two independent processes (Saraoglu and Detzler, 2002). First, a variety of optimal risky portfolios are constructed using the input data from the results of market research, such as assets' expected returns, standard deviations and correlations. For the same input data, these optimal risky portfolios are identical for all investors regardless of their risk tolerance. Second, the optimal complete portfolio is determined by considering the investor's preference and risk tolerance level.

Risk tolerance is a person's emotional and financial capacity to tolerate the ups and downs of the investment market. Risk tolerances vary from person to person, and from period to period. Some are associated with personality factors, while others are related to the stages in the investor's life cycle. Financial advisors often ask individual investors to fill out a questionnaire whose primary purpose is to assess the investors' risk tolerance as well as time horizon, investment experience, and financial status. Each question in the questionnaire is given a score and the scores are accumulated. A recommendation of asset allocation is typically based on the accumulated score of an investor's responses using .rules of thumb. For example, portfolio allocation scoring system (PASS), the first 
published risk tolerance questionnaire developed by Droms in early 1980s (see Droms and Strauss (2003)), is such a system that the more aggressive the investor's return objectives and the higher the investor's risk tolerance, the more scores will be assigned to the investor. The higher the score, the more aggressive is the asset allocation plan. Other questionnaires, such as ones developed by New York Institute of Finance (2003) and Vanguard Group (2003), provide similar functions.

PASS and most existing questionnaire-based measurements use the accumulated score to choose an asset allocation plan according to pre-designed investment categories. No doubt, these methods provide practical convenience to investment companies and financial advisors. However, it is not realistic that a definitive asset allocation plan can be obtained by a questionnaire itself (Droms and Strauss, 2003). Also, using a scoring system to measure risk tolerance may not have theoretic support that allows the value of risk tolerance to be incorporated into an optimization model such as Model 2.2 directly.

In order to model efficient asset allocation problems, Bolster and Warrick (2000) used the risk acceptance parameter (RAP) to reflect the scores. A higher RAP indicates greater risk tolerance. They estimated the value of risk tolerance by assigning a RAP value according to the responses of an investor made to a designed questionnaire. Using this method, the value of risk tolerance can be incorporated directly into an optimization model. However, there is a drawback of using this method. Although the response to the questionnaire reflects the investor's risk attitude, it is not necessarily also true for the value of RAP assigned, simply because there is no direct link between these two numbers. The RAP reflects just the advisor's, instead of the investor's, subjective attitude because the value of risk tolerance heavily depends on the selection of reference portfolios. Moreover, in a multiple objective modeling context, since the performance of asset classes varies significantly in terms of different objectives, using rules of thumb to assign the risk tolerance value is not useful.

\subsection{Theoretic Foundation for the Proposed Method}

The proposed method is based on Sharpe's (1987) utility function definition and risk tolerance definition. Sharpe assumed an investor's objective was to maximize the expected utility of wealth. As discussed in Section 2.3, the utility function can be illustrated by 
such a formula:

$$
U=E\left(R_{P}\right)-\frac{\sigma_{P}^{2}}{t}
$$

where:

$U=$ utility of portfolio for an given investor;

$\mathrm{E}\left(\mathrm{R}_{\mathrm{P}}\right)=$ expected return of portfolio;

$\sigma_{\mathrm{P}}=$ standard deviation of return of portfolio;

and $t=$ the investor's risk tolerance.

In this formula, an investor's risk tolerance is assumed constant, and defined as the added variance for per unit of added expected return, providing the same utility for the investor (Maginn and Tuttle, 1990, p. 7-9). A method for measuring risk tolerance can be derived from this formula and is described below.

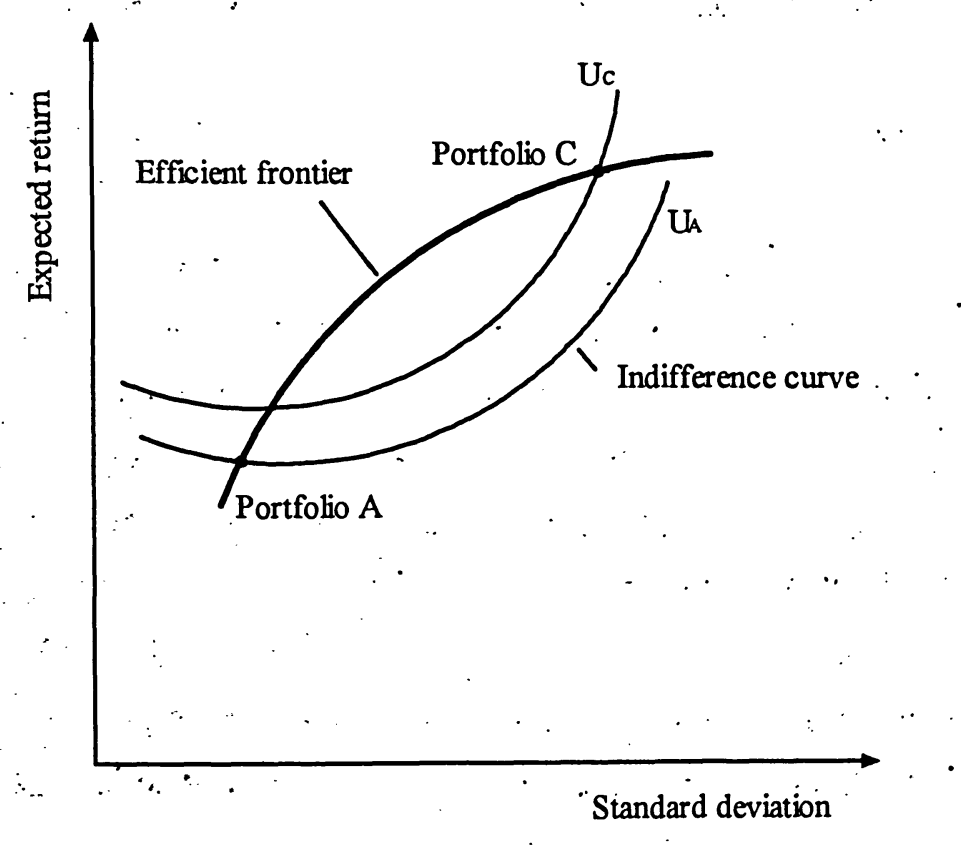

Figure 4.1: Efficient frontier and indifference curves

Along the efficient frontier showed in Figure 4.1, each portfolio, with given expected return $E\left(R_{P}\right)$, has associated standard deviation $\sigma_{\mathrm{P}}$, which is minimal among all available portfolio mix. However, different optimal portfolio mix may provide different utility to the given investor. In Figure 4.1 , portfolio $A$, with expected return $\mathrm{E}\left(\mathrm{R}_{\mathrm{A}}\right)$ and standard 
deviation $\sigma_{A}$, provides the investor with utility value of $U_{A}$; portfolio $C$; with expected return $E\left(R_{C}\right)$ and standard deviation $\sigma_{C}$, provides the investor with utility value of $U_{C}$. Every portfolio in the investor's indifference curve provides the same level of utility to the given investor.

For two portfolios, $\mathrm{A}$ and $\mathrm{C}$, the exact values of utilities are unknown to both the investor and the financial advisor. However, the investor's relative degree of preference between these two portfolios can be estimated through appropriately designed procedures. If the investor's relative degree of preference between portfolio $\mathrm{A}$ and portfolio $\mathrm{C}$ is denoted by $\dot{k}$, then $k$ can be expressed as:

$$
k=\frac{U_{A}}{U_{C}}
$$

From utility definition formula (4.2.1), the utilities of portfolio $\mathrm{A}$ and portfolio $\mathrm{C}$ are written as:

$$
U_{A}=E\left(R_{A}\right)-\frac{\sigma_{A}^{2}}{t}
$$

and

$$
U_{c}=E\left(R_{C}\right)-\frac{\sigma_{C}^{2}}{t}
$$

From (4.2.2), (4.2.3), and (4.2.4), we can derive the following formula:

$$
t=\frac{\sigma_{A}^{2}-k \sigma_{C}^{2}}{E\left(R_{A}\right)-k E\left(R_{C}\right)}
$$

Formula (4.2.5) indicates that an investor's risk tolerance can be defined as the ratio of added portfolio variances to added portfolio returns, considering the investor's relative degree of preference between two reference portfolios. Therefore, if the characteristics of two reference portfolios, $\mathrm{E}\left(\mathrm{R}_{\mathrm{A}}\right), \sigma_{\mathrm{A}}$ and $\mathrm{E}\left(\mathrm{R}_{\mathrm{C}}\right), \sigma_{\mathrm{C}}$, as well as the value of $k$, are known, the value of $t$ can be determined. In the next sections of this chapter, methods to obtain the appropriate reference portfolios and to estimate the value of $k$ are proposed. The investor selects his own reference portfolios from the candidates provided by the financial advisor. His relative degree of preference between the two reference portfolios is evaluated through the AHP technique. 


\subsection{Selection of Reference Portfolios}

In order to measure an investor's risk tolerance, the financial advisor should first obtain two reference portfolios with known characteristics. These two portfolios can be found along the efficient frontier if we assume that the investor is rational, that is, he always prefers the higher return and less risk. The selection. of reference portfolios significantly affects the value of risk tolerance. In order to let the investor make a better and more clear comparison, one of the two portfolios should be considered as very conservative, and the other should be considered as very aggressive. These two portfolios, A and C, can be described as follows:

\section{Portfolio A:}

An efficient portfolio that the investor believes is very conservative. It has a small expected return and a small variance.

Portfolio C:

An efficient portfolio that the investor believes is very aggressive. It has a large expected return and a large variance.

There are two options for the selection procedure. The first one is the advisor provides the reference portfolios to the investor. The second one is the investor selects own reference portfolios. The first option is more convenient but the investor may not think the reference portfolios are suitable to him. Because different people have different risk attitudes towards a same portfolio, the "aggressiveness" of reference portfolios is vague if they are not selected by an investor himself. This difference can be illustrated by an example. Assuming there are two portfolios, say, $\mathrm{A}$ and $\mathrm{C}$, which are considered conservative and aggressive respectively by the advisor. The investor is asked to choose one of them to invest in it. If investor chooses portfolio $\mathrm{C}$, he is considered "aggressive". If the investor chooses $\mathrm{A}$, he is considered "conservative". However, to the investor himself, he may think he is just choosing the "right" portfolio according to his own preference, neither "aggressive" nor "conservative". It means an investor doesn't always think in the same manner as the advisor or other people, who select the reference portfolios. Therefore, it is desirable to ask the investor to select his own reference portfolios. Considering the limited investment knowledge of an investor, in the proposed method, the advisor will provide several candidate portfolios, and the investor chooses reference portfolios from the candidates. . 


\subsection{Select the Conservative Reference Portfolio}

The expected return of a portfolio is determined by the characteristics of assets making up the portfolio. It is a weighted average of the expected returns for individual assets in the portfolio. Usually people believe that, if an investor is willing to invest most of his funds to assets that have low volatility of expected returns, he is conservative; because usually the lower the volatility, the lower is the return. Since the advisor only helps an investor select reference portfolio instead of making judgment for him, a reasonable number of candidate portfolios should be provided. A procedure based on a heuristic method is used to select the conservative reference portfolio.

\subsubsection{Heuristic Method}

The following heuristic can be used to select the conservative reference portfolio A:

A very conservative portfolio $=$ The first portfolio, from the top down in a list of portfolio (called $A_{c}$ ) which the specified investor believes it is investable.

Here $A_{c}$ denotes the set of the efficient portfolios that most people believe they are very conservative. The portfolios in $A_{c}$ are sorted in terms of their relative conservativeness and the top portfolio in the list is the most conservative.

The statement means the investor believes the reference portfolio $\mathrm{A}$ is investable in terms of his preference, although the possibility to invest in it is low because it is too conservative to him. Based on the above heuristic, a procedure to select reference portfolio A is described as follows.

Step 1: The financial advisor generates the list $A_{c}$ that includes several efficient portfolios that are usually believed (that is, according to the advisor's experience) as very conservative. The global minimum variance portfolio $\mathrm{K}$, described in Chapter 2 , is the low bound of this list.

Step 2: The financial advisor sorts these portfolios in $\mathrm{A}_{\mathrm{c}}$ in decreasing order according to their relative conservativeness. The most conservative portfolio is sorted at the top of the list. 
Step 3: The financial advisor asks the investor to judge if it is possible for him to invest in one of the portfolios, starting from the top of the list $A_{c}$. If the answer is no, ask the investor to make judgment for the next portfolio. This procedure continues until the investor makes a positive answer.

\subsubsection{The Global Minimum Variance Portfolio}

The global minimum variance portfolio $\mathrm{K}$ is considered as the most conservative portfolio. It can be used as a good starting point for the advisor to generate candidate reference portfolios.

All portfolios along the efficient frontier have such a property that when the expected return decreases, its standard deviation also decreases. Portfolios on both sides of the global minimum variance portfolio $K$ have larger variance than $K$. As shown in Figure 4.2, when the expected return of a portfolio continues to decrease ard once passes $K$, the portfolio's variance no longer decreases but increases. It is said this portfolio becomes inefficient. Based on this property, a heuristic algorithm is developed to find the portfolio $\mathrm{K}$, This algorithm is to search along the efficient frontier with decreasing expected returns until the variance of one portfolio is found to be larger than its predecessor.

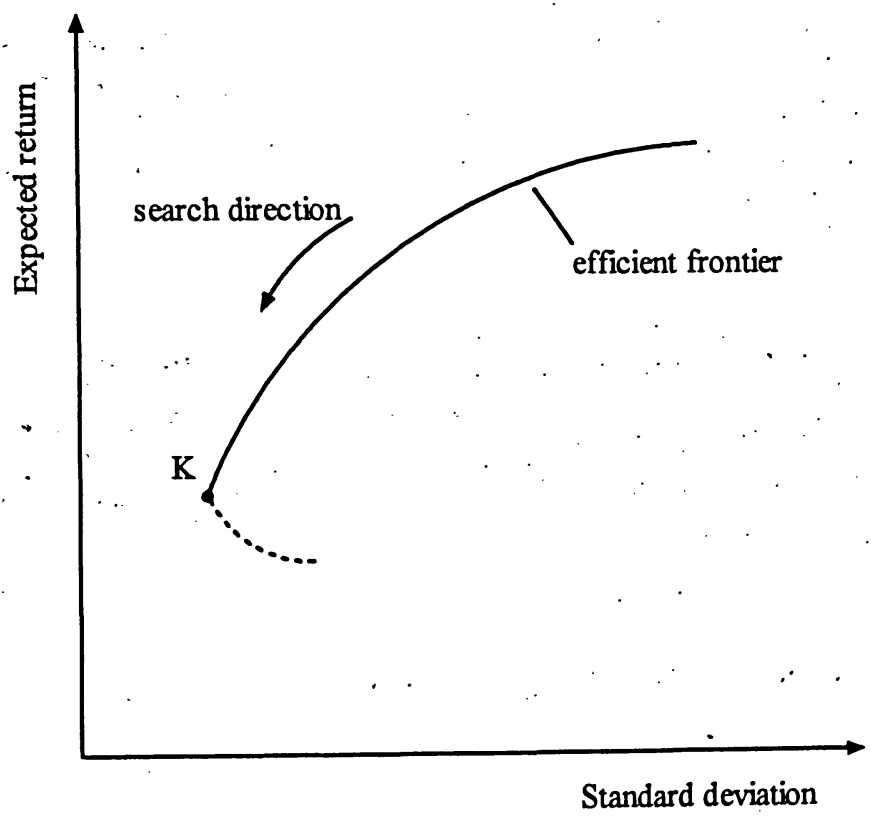

Figure 4.2: Search for the global minimum variance portfolio 


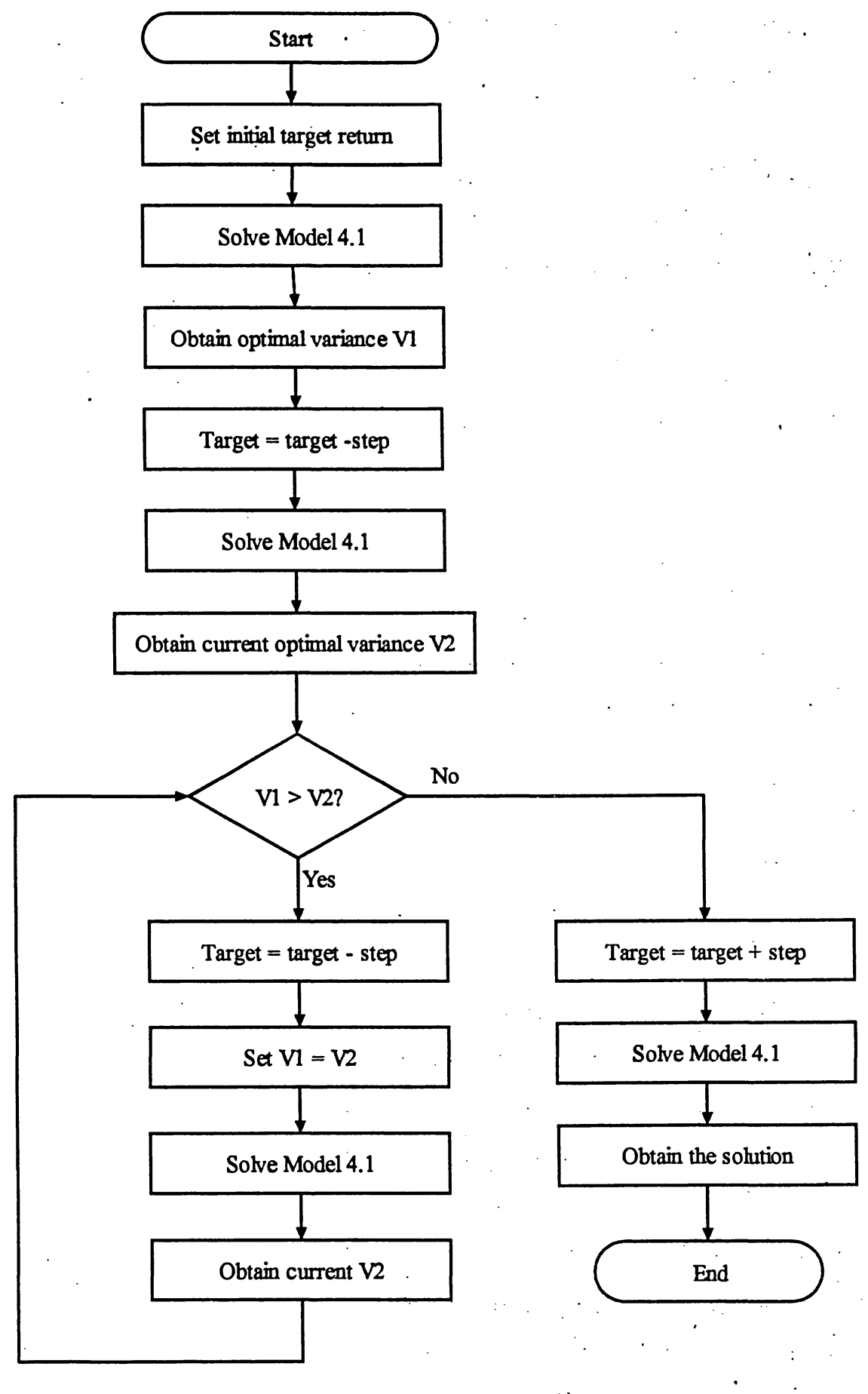

Figure 4.3: Flowchart for finding the global mimimum variance portfolio

The flowchart based on the above algorithm is shown in Figure 4.3. The procedure is implemented as follows. First, specify an initial target return, solve the mean-variance 
optimization model (denoted as Model 4.1) and obtain a minimum variance V1. Then decrease the target return value by one step length, solve Model 4.1 again, and obtain current minimum variance V2. Compare V1 with V2. If V1 is larger than the current variance $\mathrm{V} 2$, continue the search. If $\mathrm{V} 2$ is larger than $\mathrm{V} 1$, this means the predecessor of the current portfolio is the global minimum variance portfolio.

Model 4.1 is a quadratic programming model used to find the optimal value of portfolio variance associated with given expected target return. This model is an alternative formulation of Markvitiwz model (Model 2.1). Its general formulation is written as follows:

Model 4.1:

Objective:

$$
\text { Minimize } \sigma_{P}^{2}=\sum_{i=1}^{N} x_{i}^{2} \sigma_{i}^{2}+\sum_{\substack{i=1 \\ j}}^{N} \sum_{\substack{j=1 \\ j \neq i}}^{N} x_{i} \dot{x}_{j} \rho_{i j} \sigma_{i} \sigma_{j}
$$

subject to:

$$
\begin{aligned}
& E\left(R_{P}\right)=\sum_{i=1}^{N} x_{i} E\left(R_{i}\right) \\
& \sum_{i=1}^{N} x_{i}=1 \\
& x_{i} \geq 0 \quad i=1, \ldots, N
\end{aligned}
$$

where: $\mathrm{E}\left(\mathrm{R}_{\mathrm{P}}\right)=$ expected return of portfolio;

$\sigma_{\mathrm{P}}=$ standard deviation of return of portfolio;

$\mathrm{E}\left(\mathrm{R}_{i}\right)=$ expected return for asset class $i$;

$\sigma_{i}=$ standard deviation of return of asset class $i$;

$\rho_{i j}=$ correlation coefficient between asset class $i$ and $j$;

$\mathrm{N}=$ the number of asset classes;

and $\mathrm{x}_{i}=$ proportion of portfolio invested in asset class $i$.

Computer programs based on General Algebraic Modeling System (GAMS, developed by GAMS Development Corporation) language are written to implement the algorithm for 
finding the global minimum portfolio for each objective (Appendices 1.1, 2.1, 3.1, 4.1, 5.1, and 6.1). The inputs for each program are the initial target value of expected return and the step length. The outputs are the characteristics of the portfolio $\mathrm{K}$, including the expected return, standard deviation, and optimal weight of each asset class making up the portfolio. The results for the hypothetical investment example using data of Table 3.1 and Table 3.2 are shown in Table 4.1.

Table 4.1: The global minimum variance portfolio for each objective

\begin{tabular}{ccccccccc}
\hline & Return & STD & \multicolumn{6}{c}{ Fraction of assets making up the portfolio } \\
\cline { 4 - 9 } Objective & $\%$ & $\%$ & LaStock & SmStock & LGBond & LCBond & T-Bill & RealEst \\
\hline INR & 3.9 & 3.161 & 0.025 & 0.000 & 0.080 & 0.000 & 0.895 & 0.000 \\
CA & 0.0 & 0.996 & 0.007 & 0.004 & 0.000 & 0.000 & 0.989 & 0.000 \\
CRI & 4.5 & 1.629 & 0.246 & 0.000 & 0.230 & 0.000 & 0.355 & 0.169 \\
ATR & 2.2 & 1.739 & 0.016 & 0.004 & 0.083 & 0.000 & 0.897 & 0.000 \\
PP & 1.0 & 4.100 & 0.042 & 0.000 & 0.125 & 0.000 & 0.833 & 0.000 \\
LD & 0.0 & 0.000 & 0.000 & 0.000 & 0.000 & 0.000 & 0.000 & 1.000 \\
\hline
\end{tabular}

\subsubsection{Generating the List $\mathrm{A}_{\mathrm{c}}$ of the Candidate Portfolios}

A simple algorithm is used to generate the list $\mathrm{A}_{c}$ of the candidate portfolios. The financial advisor first specifies an initial small target return for one portfolio. This value can be the expected return of the global minimum portfolio $\mathrm{K}$. But it is up to the advisor's choice if the portfolio $\mathrm{K}$ is believed to be too conservative and he would like to limit the size of the list. After generating the first portfolio, increase the target return by one step length, solve Model 4.1 again, and obtain the second portfolio. Continue this procedure until the required size of candidate portfolios is met. The flowchart based on this algorithm is shown in Figure 4.4. GAMS programs are written to implement the algorithm for generating the list of candidate conservative portfolios for each objective (see Appendices 1.2, 2.2, 3.2, 4.2, 5.2, and 6.2). The inputs for each program are the initial target value of expected return, the step length as well as the required size of list $n$, The outputs are the characteristics of each portfolio, including the expected return, standard deviation, and optimal weight of each asset class making up the portfolio. Sorting the portfolios in the list is automatically completed using the above algorithm. 

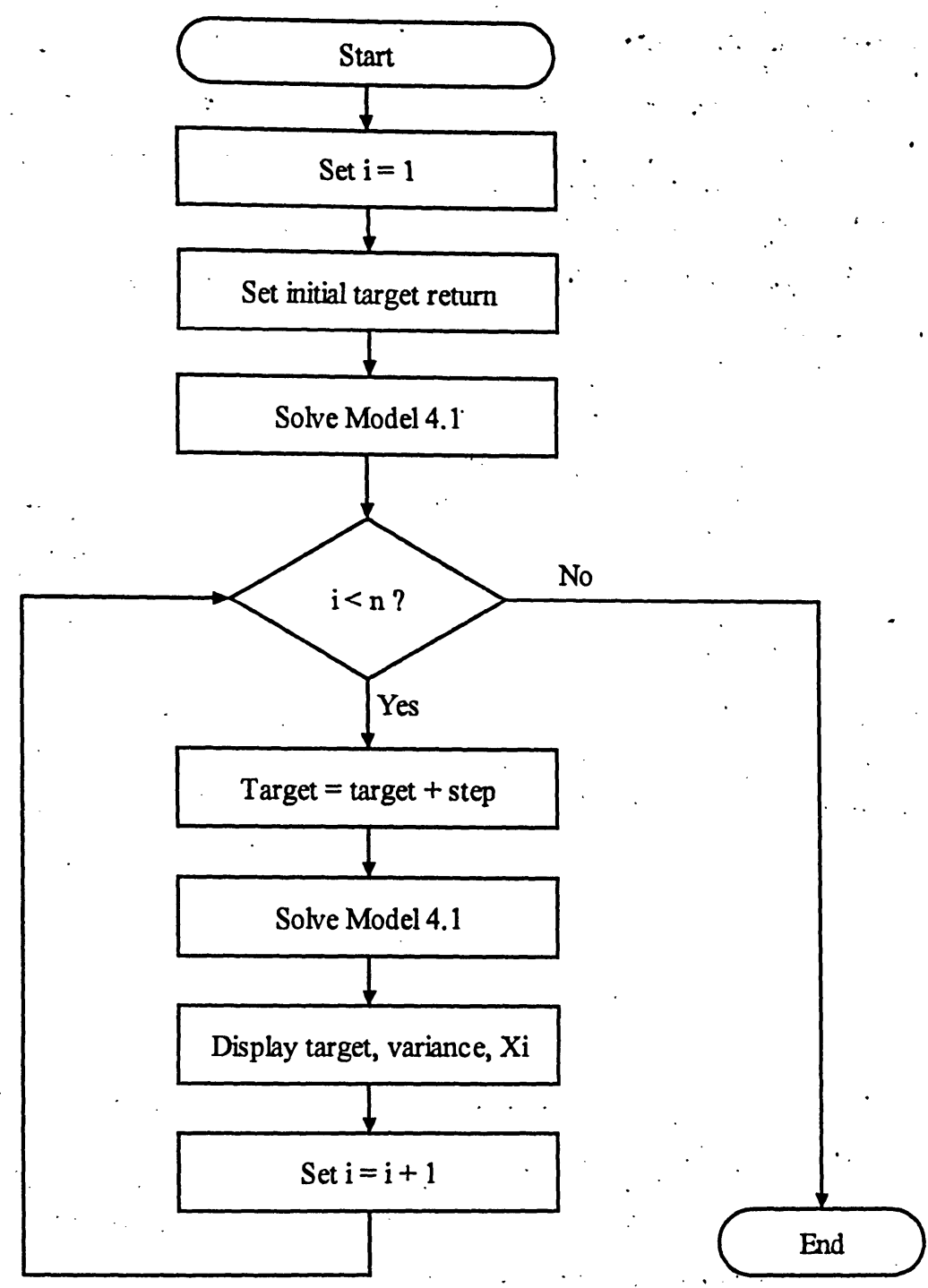

Figure 4.4: Generating the list of the conservative reference portfolios

\subsubsection{Select the Reference Portfolio A Using Questionnaires}

The investor selects his own reference portfolio from the candidate portfolio list $A_{c}$ provided by the advisor. This procedure can be implemented by using questionnaires. Normally investors consider the portfolio return and risk in terms of dollar value, so presenting one portfolio to investors in dollar value description may be more straightforward. If the return distribution is assumed to follow approximately the normal distribution, then the chance that the outcome value of expected return will fall in $[E(R)$ 
$\pm \sigma]$ is $68 \%$ (approximately two of three times), and the chance that the outcome value of expected return will fall in $[E(R) \pm 3 \sigma]$ is $99.7 \%$. If the extreme value is considered as worse or best case, the following description of portfolio can be used when designing a questionnaire.

\section{The description of portfolio A:}

You have an available capital of $\$ \mathrm{~V}$. If you invest it in portfolio $\mathrm{A}$, the average net return of this investment by the end of investment period will be $\$ V \times E\left(R_{A}\right)$. Two out of three times that the net return of this investment will come out to be between $\$ V \times$ $\left[E\left(R_{A}\right)-\sigma_{A}\right]$ and $\$ V \times\left[E\left(R_{A}\right)+\sigma_{A}\right]$ However, in the worse case, the investment will lose $\$ V \times\left[E\left(R_{A}\right)-3 \sigma_{A}\right]$, and in the best case the investment will gain $\$ V \times\left[E\left(R_{A}\right)+\right.$ $\left.3 \sigma_{\mathrm{A}}\right]$;

A sample questionnaire, designed for selecting the conservative reference portfolio in terms of the total nominal return objective, is shown in Figure 4.5. If the response from the investor is "absolutely not", the advisor presents other portfolios in the candidate list one by one. If the investor's answer is "it is possible", set this portfolio as the reference portfolio A.

\subsection{Select the Aggressive Reference Portfolio}

The expected return of a portfolio is limited and must fall between the highest and lowest expected returns of the component individual assets. Usually people believe that, if an investor is willing to invest most of his funds to assets that have highest attainable expected returns, he is aggressive; because usually the higher the return, the higher is the risk. But for an individual investor, his attitude towards the definition of aggressiveness may vary significantly. For example, some investors may think investing in stocks is always a very risky action and always try to avoid it. Therefore, the maximum return portfolio is not necessarily the right aggressive reference portfolio for a specific investor. A procedure based on a heuristic method is used to select the aggressive reference portfolio. 
This questiomaire is designed for an investor to select the reference conservative portfolio in terms of the total nominal return objective: Based on the description of this portfolio, please answer the question provided below.

Description of portfolio A (based on $\$ 100,000$ funds)

\begin{tabular}{|c|c|c|c|c|c|c|}
\hline $\begin{array}{l}\text { Expected } \\
\text { Return( \%) } \\
\end{array}$ & $\begin{array}{l}\text { STD } \\
(\%) \\
\end{array}$ & $\begin{array}{c}\text { Average net } \\
\text { return }(\$)\end{array}$ & $\frac{2 \text { out of } 3 \text { tim }}{\text { Low end }(\$)}$ & $\begin{array}{c}\text { S net return range } \\
\text { High end (\$) }\end{array}$ & $\begin{array}{r}\text { e Worse case } \\
\text { net return(\$) }\end{array}$ & $\begin{array}{l}\text { Best case } \\
\text { net return(\$) }\end{array}$ \\
\hline 4 & 3.162 & 4,000 & 838 & 7,162 & $-5,486$ & 13,486 \\
\hline
\end{tabular}

\section{Question:}

You have an available capital of $\$ 100,000$. Would you like to invest in this portfolio?

Your answer is:

: 1 . Absolutely not.' 2. It is possible.

Figure 4.5: Example questionmaire for selecting the conservative reference portfolio

\subsubsection{Heuristic Method}

The heuristic used for selecting the aggressive reference portfolio can be expressed as follows:

A very aggressive portfolio $=$ The first portfolio, from the top down in set $C_{a}$ that the specified investor believes it is investable.

Here $C_{a}$ denotes the set of efficient portfolios that most people believe they are very aggressive. The portfolios in $\mathrm{C}_{\mathrm{a}}$ are sorted in terms of their relative aggressiveness and the top portfolio in the list is the most aggressive.

The statement means the investor believes the reference portfolio $\mathrm{C}$ is investable in terms of his preference, although the possibility to invest in it is low because it is too aggressive to him. Based on the above heuristic, a procedure used to select reference the reference portfolio $\mathrm{C}$ is suggested as follows: 
Step 1: The financial advisor generates the list $C_{a}$ that includes several efficient portfolios that are usually believed (that is, according to the advisor's experience) as very aggressive. The maximum return portfolio $\mathrm{Q}$, described in Chapter 2, is the upper bound of this list.

Step 2: The financial advisor sorts the portfolios in $\mathrm{C}_{\mathrm{a}}$ in decreasing order according to their relative aggressiveness. The most aggressive portfolio is sorted at the top of the list.

Step 3: The financial advisor asks the investor to judge if it is possible for him to invest in one of the portfolios, starting from the top of the list. If the answer is no, ask the investor to make judgment for the next portfolio. This procedure continues until the investor makes a positive answer.

\subsubsection{The Maximum Return Portfolio}

The maximum return portfolio $Q$ is considered as the most aggressive efficient portfolio: It can be used as a good starting point for the advisor to generate candidate aggressive reference portfolios.

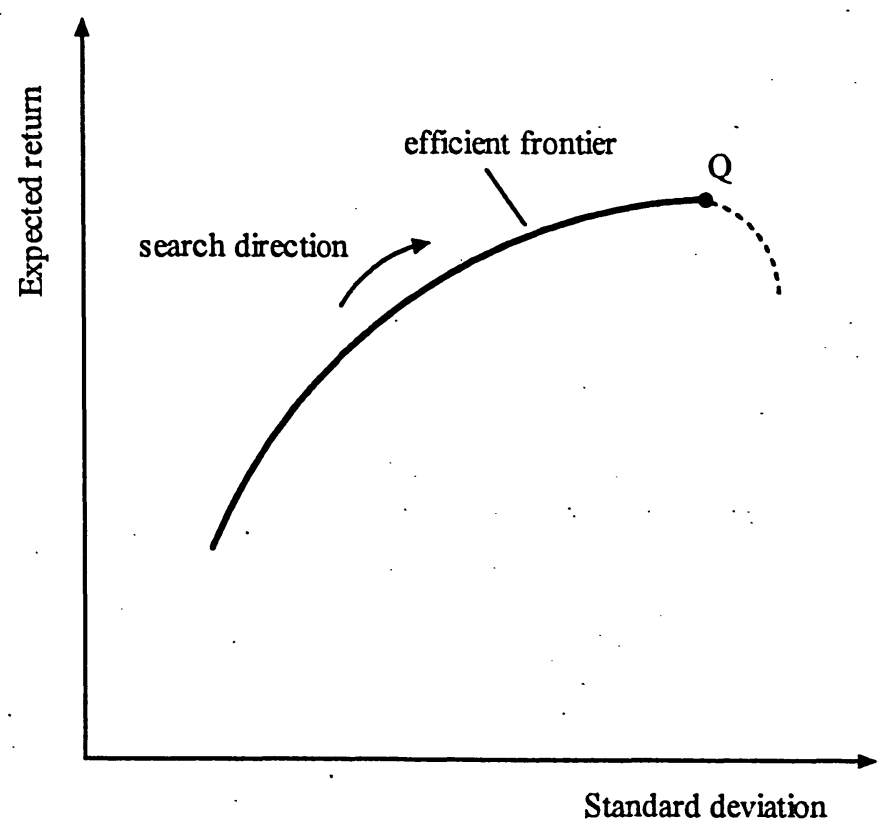

Figure 4.6: Search for the maximum return portfolio 
All portfolios along the efficient frontier have such a property that when the standard deviation increases, expected return also increases. Portfolios on both sides of the maximum return portfolio $Q$ have smaller expected return than $Q$. As shown in Figure 4.6, when the standard deviation of a portfolio continues to increase and once passes $Q$, the portfolio's return no longer increases but decreases. It is said this portfolio becomes inefficient. Based on this property, a heuristic algorithm is developed to find the portfolio $\mathrm{Q}$. This algorithm is to search along the efficient frontier with increasing variances until the expected return of one portfolio is found to be smaller than its predecessor.

The flowchart based on the above algorithm is shown in Figure 4.7. The procedure is implemented as follows. First, specify an initial target variance, solve the mean-variance optimization model as given in Model 2.1, and obtain an optimal return R1. Then increase the target variance value by one step length, solve Model 2.1 again, and obtain the current optimal return R2. Compare $R 1$ with $R 2$. If $R 1$ is smaller than the current optimal return $\mathrm{R} 2$, continue the search. If $\mathrm{R} 1$ is larger than $\mathrm{R} 2$, this means the predecessor of the current portfolio is the maximum return portfolio.

GAMS programs are written to implement the algorithm for finding the maximum return portfolio for each objective (see Appendices 1.3, 2.3, 3.3, 4.3, 5.3, and 6.3). The inputs for each program are the initial target value of standard deviation and the step length. The outputs are the characteristics of the portfolio $\mathrm{Q}$, including the expected return, standard deviation, and optimal weight of each asset class making up the portfolio. The results for the hypothetical investment example using data of Table 3.1 and Table 3.2 are shown in Table 4.2.

Table 4.2: The maximum return portfolio for each objective

\begin{tabular}{ccccccccc}
\hline & Return & STD & \multicolumn{6}{c}{ Fraction of assets making up the portfolio } \\
\cline { 4 - 9 } Objective & $\%$ & $\%$ & LaStock & SmStock & LGBond & LCBond & T-Bill & RealEst \\
\hline TNR & 17.8 & 35.6 & 0.000 & 1.000 & 0.000 & 0.000 & 0.000 & 0.000 \\
CA & 12.8 & 27.8 & 0.000 & 1.000 & 0.000 & 0.000 & 0.000 & 0.000 \\
CRI & 5.9 & 3.5 & 0.000 & 0.000 & 0.000 & 1.000 & 0.000 & 0.000 \\
ATR & 13.3 & 26.5 & 0.000 & 1.000 & 0.000 & 0.000 & 0.000 & 0.000 \\
PP & 14.3 & 24.9 & 0.000 & 1.000 & 0.000 & 0.000 & 0.000 & 0.000 \\
LD & 13.4 & 26.7 & 0.000 & 1.000 & 0.000 & 0.000 & 0.000 & 0.000 \\
\hline
\end{tabular}




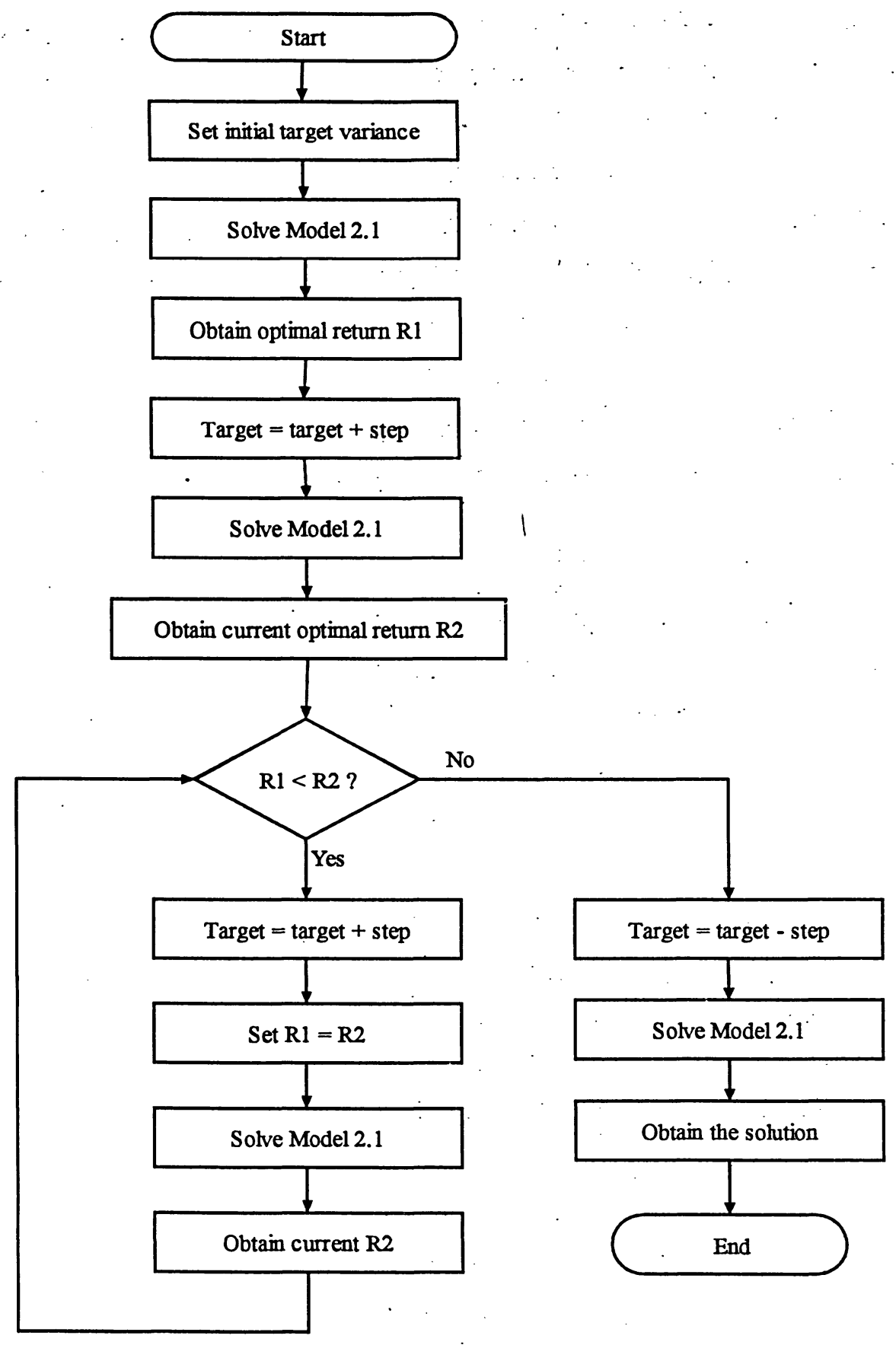

Figure 4.7: Flowchart for finding the maximum return portfolio *

\subsubsection{Generating the List $C_{a}$ of the Candidate Portfolios}

The algorithm used to generate the candidate portfolio list $C_{a}$ is similar to the one used to 
generate the candidate conservative portfolio list $\mathrm{A}_{c}$. The financial advisor first specifies an initial large target return for one portfolio. This initial value can be the expected return of the maximum return portfolio $Q$. But it is* up to the advisor's choice if the portfolio $Q$ is believed to be too aggressive and he would like to limit the size of the list. After generating the first portfolio, decrease the target return by one step length, solve Model 4.1 again, obtain the second portfolio. The process stops once the required size of candidate portfolios is reached. The flowchart based on this algorithm is shown in the Figure 4.8. GAMS programs similar to ones given in Appendices 1.2, 2.2, 3.2, 4.2, 5.2, and 6.2 are used to implement the algorithm for generating the candidate aggressive portfolio list for each objective. The inputs for each program are the initial target value of expected return, the step length, as well as the required size of the list $n$. The outputs are the characteristics of each portfolio, including the expected return, standard deviation, and optimal weight of each asset class making up the portfolio. Sorting the portfolios in the list is automatically achieved using the above algorithm.

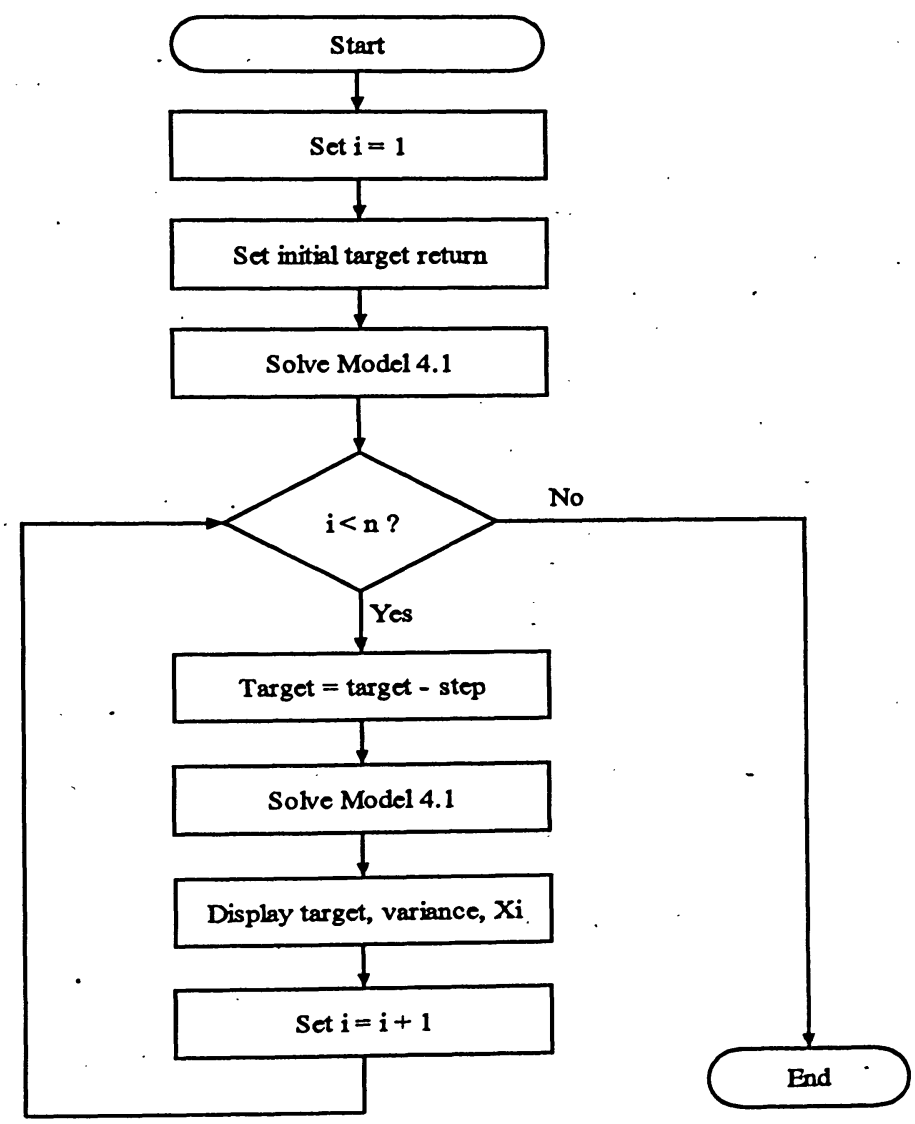

Figure 4.8: Generating the aggressive reference portfolio list 


\subsubsection{Select the Reference Portfolio C Using Questionnaires}

The questionnaires used to select the aggressive reference portfolio $\mathrm{C}$ are similar to those for selecting the conservative reference portfolio A. A sample questionnaire, designed for selecting the aggressive reference portfolio for the total nominal return objective, is shown in Figure 4.9. If the response from the investor is "absolutely not", the advisor presents other portfolios in the candidate list one by one. If the investor's answer is "it is possible", set this portfolio as the reference portfolio $\mathrm{C}$.

This questionnaire is designed for an investor to select the reference aggressive portfolio in terms of the total nominal return objective. Based on the description of this portfolio, please answer the question provided below.

Description of portfolio C (based on $\$ 100,000$ funds)

\begin{tabular}{|c|c|c|c|c|c|c|}
\hline $\begin{array}{l}\text { Expect } \\
\text { Return }\end{array}$ & $\begin{array}{l}\text { STD } \\
(\%)\end{array}$ & $\begin{array}{l}\text { Average net } \\
\text { return (\$) }\end{array}$ & $\frac{2 \text { out of } 3 \text { times }}{\text { Low end }(\$)}$ & $\frac{\text { net return range }}{\text { High end }(\$)}$ & $\begin{array}{l}\text { Worse case } \\
\text { net return(\$) }\end{array}$ & $\begin{array}{c}\text { Best case } \\
\text { net return(\$) }\end{array}$ \\
\hline 13 & 20.30 & 13,000 & $-7,300$ & 33,300 & $-47,900$ & 73,900 \\
\hline
\end{tabular}

\section{Question:}

You have an available capital of $\$ 100,000$. Would you like to invest in this portfolio?

Your answer is:

1. Absolutely not. 2. It is possible.

Figure 4.9: Example questionnaire for selecting the aggressive reference portfolio

Assuming that the hypothetical investor has selected the conservative reference portfolio $\mathrm{A}$ and aggressive reference portfolio $\mathrm{C}$ in terms of each objective. These portfolios are summarized in Table 4.3 
Table 4.3: Reference portfolios for each objective

\begin{tabular}{ccccccccccc}
\hline & & Reference & $\mathrm{E}(\mathrm{R})$ & $\sigma$ & \multicolumn{5}{c}{ Fractions of assets making up the portfolio } \\
\cline { 5 - 12 } Objective & portfolio & $(\%)$ & $(\%)$ & LaStock & SmStock & LCBond & LGBond & T-Bill & RealEst \\
\hline TNR & $\mathrm{A}$ & 4.0 & 3.161 & 0.030 & 0.004 & 0.000 & 0.087 & 0.879 & 0.000 \\
& $\mathrm{C}$ & 13.0 & 20.30 & 0.237 & 0.426 & 0.000 & 0.000 & 0.000 & 0.335 \\
\hline CA & $\mathrm{A}$ & 3.0 & 6.165 & 0.130 & 0.130 & 0.000 & 0.000 & 0.548 & 0.192 \\
& $\mathrm{C}$ & 10.0 & 20.78 & 0.049 & 0.710 & 0.000 & 0.000 & 0.000 & 0.241 \\
\hline CRI & $\mathrm{A}$ & 4.5 & 1.629 & 0.246 & 0.000 & 0.000 & 0.230 & 0.355 & 0.169 \\
& $\mathrm{C}$ & 5.9 & 3.500 & 0.000 & 0.000 & 0.000 & 1.000 & 0.000 & 0.000 \\
\hline ART & $\mathrm{A}$ & 3.0 & 2.278 & 0.065 & 0.011 & 0.142 & 0.010 & 0.676 & 0.096 \\
& $\mathrm{C}$ & 10.0 & 16.67 & 0.270 & 0.487 & 0.000 & 0.000 & 0.000 & 0.243 \\
\hline PP & $\mathrm{A}$ & 2.0 & 4.564 & 0.083 & 0.021 & 0.000 & 0.191 & 0.628 & 0.077 \\
& $\mathrm{C}$ & 9.0 & 18.20 & 0.257 & 0.354 & 0.000 & 0.000 & 0.000 & 0.388 \\
\hline LD & $\mathrm{A}$ & 3.0 & 2.271 & 0.033 & 0.016 & 0.025 & 0.064 & 0.554 & 0.307 \\
& $\mathrm{C}$ & 10.0 & 15.284 & 0.519 & 0.176 & 0.305 & 0.000 & 0.000 & 0.000 \\
\hline
\end{tabular}

\subsection{Relative Degree of Preference between Reference Portfolios}

After the reference portfolios are chosen, the next step is to obtain the investor's. relative preference of two portfolios for each objective. Because both portfolios $\mathrm{A}$ and $\mathrm{C}$ are not the desired portfolio for the investor, he is not asked to make direct comparison between portfolios A and C. Instead, another reference portfolio, the investor's desired portfolio, is employed as a medium to derive the relative preference between portfolio $\mathrm{A}$ and $\mathrm{C}$. This portfolio, denoted as portfolio $\mathrm{B}$, is the one that the investor will most likely choose, but it is not necessary for him to know the exact characteristics of this portfolio.

Questionnaires, such as the sample questionnaire shown in Figure 4.10, are designed to assess the investor's relative degree of preference. In these questionnaires; the investor is asked to express his preference between portfolio $\mathrm{B}$ and $\mathrm{A}$, and his preference between $\mathrm{B}$ and $\mathrm{C}$, in terms of each objective. The scale used in this questionnaire is similar to the one suggested by Saaty (1980). 
This questionnaire is designed for the purpose of assessing your preference over the reference portfolios in terms of the total nominal return objective. You have selected Portfolio $A$ as a conservative portfolio and selected Portfolio $\mathrm{C}$ as the aggressive portfolio. The portfolio, say, B, is your most desired portfolio. Please use the.suggested scale to represent your preference between two portfolios in terms of the total nominal return objective. The former portfolio mentioned in each question is referred as portfolio $\mathrm{I}$, and the latter portfolio is referred as portfolio II

\section{Description of portfolios A and C}

\begin{tabular}{|c|c|c|c|c|c|c|c|}
\hline & \multirow{2}{*}{$\begin{array}{l}\text { Expected } \\
\text { Return(\%) }\end{array}$} & \multirow{2}{*}{$\begin{array}{l}\text { STD } \\
(\%)\end{array}$} & \multirow{2}{*}{$\begin{array}{l}\text { Average net } \\
\text { return (\$) }\end{array}$} & \multicolumn{2}{|c|}{2 out of 3 times net return range } & \multirow{2}{*}{$\begin{array}{l}\text { Worse Case } \\
\text { net return }(\$)\end{array}$} & \multirow{2}{*}{$\begin{array}{l}\text { Best Case } \\
\text { net return }(\$)\end{array}$} \\
\hline & & & & Low end (\$) & Figh end (\$) & & \\
\hline \multirow[t]{2}{*}{ Portfolio A } & 4 & 3.162 & 4,000 & 838 & 7,162 & $-5,486$ & 13,486 \\
\hline & \multicolumn{7}{|c|}{$\begin{array}{l}\text { Fractions of assets making up portfolio A } \\
\text { LaStock } 0.030, \text { SmStock } 0.004, \text { LGBond } 0.087 \text {, LCBond } 0.000 \text {, TBill } 0.879 \text {, RealEst } 0.000\end{array}$} \\
\hline \multirow[t]{2}{*}{ Portfolio C } & 13 & 20.30 & 13,000 & $-7,300$ & 33,300 & $-47,900$ & 73,900 \\
\hline & \multicolumn{7}{|c|}{$\begin{array}{c}\text { Fractions of assets making up portfolio C: } \\
\text { LaStock } 0.237, \text { SmStock } 0.428 \text {, LGBond } 0.000 \text {, rCBond } 0.000 \text {, TBill } 0.000 \text {, RealEst } 0.335\end{array}$} \\
\hline
\end{tabular}

Suggested scale for pairwise comparison assessments

\begin{tabular}{cl}
\hline 1 & If portfolio I and portfolio II are judged to be equally preferred \\
3 & If portfolio I is judged to be slightly more preferred over portfolio II \\
5 & If portfolio I is judged to be moderately more preferred over portfolio II \\
7 & If portfolio I is judged to be strongly more preferred over portfolio II \\
9 & If portfolio I is judged to be extremely more preferred over portfolio II \\
$2,4,6,8$ & If intermediate values between two adjacent judgments are needed \\
\hline $1 / 3$ & If portfolio II is judged to be slightly more preferred over portfolio I \\
$1 / 5$ & If portfolio II is judged to be moderately more preferred over portfolio I \\
$1 / 7$ & If portfolio II is judged to be strongly more preferred over portfolio I \\
$1 / 9$ & If portfolio II is judged to be extremely more preferred over portfolio I \\
$1 / 2,1 / 4,1 / 6,1 / 8$ & If intermediate values between two adjacent judgments are needed \\
\hline
\end{tabular}

\section{Questions:}

1. Do you think which scale best describes your relative preference between Portfolio B and Portfolio A? $\begin{array}{lllllllllllllllll}1 & 2 & 3 & 4 & 5 & 6 & 7 & 8 & 9 & 1 / 2 & 1 / 3 & 1 / 4 & 1 / 5 & 1 / 6 & 1 / 7 & 1 / 8 & 1 / 9\end{array}$

2. Do you think which scale best describes your relative preference between Portfolio $B$ and Portfolio $C$ ? $\begin{array}{lllllllllllllllll}1 & 2 & 3 & 4 & 5 & 6 & 7 & 8 & 9 & 1 / 2 & 1 / 3 & 1 / 4 & 1 / 5 & 1 / 6 & 1 / 7 & 1 / 8 & 1 / 9\end{array}$

Figure 4.10: Example questionnaire for assessing relative degree of preference between reference portfolios 
If the investor takes a normal action, that is, neither aggressive nor conservative according to the investor's own judgment, then most likely the answer, for example, is:

B is strongly preferred over A and B is strongly preferred over C - (Statement 1).

If the investor takes a more aggressive action, then portfolio B becomes more aggressive, and the possible answer, for example, is,

B is slightly preferred over C but strongly preferred over A -- (Statement 2).

If the investor takes a more conservative action, then portfolio B becomes more conservative, and the possible answer, for example, is,

B is slightly preferred over A but strongly preferred over C -- (Statement 3 ).

No matter what action he takes, the aggressiveness of portfolio $B$ will lie between $A$ and $C$, the lower and upper bound of the feasible portfolios.

A comparison matrix involving three portfolios, A, B, and C, car be constructed. The scale values for above three statements are entered to the matrix, the diagonal comparisons are filled with " 1 ". The resulting matrices are shown in Table 4.4.

Table 4.4: Initial comparison matrices for portfolios A, B and C

Aggressive action Normal action Conservative action

\begin{tabular}{|c|c|c|c|}
\hline & $\mathrm{A}$ & $\mathrm{B}$ & $\mathrm{C}$ \\
\hline $\mathrm{A}$ & 1 & $1 / 7$ & $?$ \\
\hline $\mathrm{B}$ & 7 & 1 & 3 \\
\hline $\mathrm{C}$ & $?$ & $1 / 3$ & 1 \\
\hline
\end{tabular}

\begin{tabular}{|c|c|c|c|}
\hline & $\mathrm{A}$ & $\mathrm{B}$ & $\mathrm{C}$ \\
\hline $\mathrm{A}$ & 1 & $1 / 7$ & $?$ \\
\hline $\mathrm{B}$ & 7 & 1 & 7 \\
\hline $\mathrm{C}$ & $?$ & $1 / 7$ & 1 \\
\hline
\end{tabular}

\begin{tabular}{|c|c|c|c|}
\hline & $\mathrm{A}$ & $\mathrm{B}$ & $\mathrm{C}$ \\
\hline $\mathrm{A}$ & 1 & $1 / 3$ & $?$ \\
\hline $\mathrm{B}$ & 3 & 1 & 7 \\
\hline $\mathrm{C}$ & $?$ & $1 / 7$ & 1 \\
\hline
\end{tabular}

The question mark "?" in the matrices means the investor doesn't have to make a direct comparison between $A$ and $C$. Instead, the consistency requirement of the comparison matrix is used to derive the answer. Or, if the investor does make a direct comparison, the consistent test still should be performed. 
A comparison matrix involving three portfolios, A, B and C can be written as:

$$
D_{3}=\left[\begin{array}{lll}
d_{A A} & d_{A B} & d_{A C} \\
d_{B A} & d_{B B} & d_{B C} \\
d_{C A} & d_{C B} & d_{C C}
\end{array}\right]
$$

A perfectly consistent matrix should satisfy the transitivity and reciprocity requirements. For matrix $D_{3}$, the following equalities should be held:

$$
\begin{aligned}
& d_{A C}=d_{A B} \times d_{B C} \\
& d_{C A}=1 / d_{A C}
\end{aligned}
$$

Using these formulae to calculate the scale values for cells assigned with "?", the matrices in Table 4.4 are transformed to those in Table 4.5.

Table 4.5: Comparison matrices for portfolios A, B and C

Aggressive action
\begin{tabular}{|c|c|c|c|}
\hline & A & B & C \\
\hline A & 1 & $1 / 7$ & $3 / 7$ \\
\hline B & 7 & 1 & 3 \\
\hline C & $7 / 3$ & $1 / 3$ & 1 \\
\hline
\end{tabular}$\quad$\begin{tabular}{|c|c|c|c|}
\hline & A & B & C \\
\hline A & 1 & $1 / 7$ & 1 \\
\hline B & 7 & 1 & 7 \\
\hline C & 1 & $1 / 7$ & 1 \\
\hline
\end{tabular}$\quad$\begin{tabular}{|c|c|c|c|c|}
\hline & A & B & C \\
\hline A & 1 & $1 / 3$ & $7 / 3$ \\
\hline B & 3 & 1 & 7 \\
\hline C & $3 / 7$ & $1 / 7$ & 1 \\
\hline
\end{tabular}

The fractions, $3 / 7$ and $7 / 3$ in matrices of Table 4.5 , don't have corresponding suggested scales, so they have to be rounded. For example, the closest suggested scale for $3 / 7$ is $1 / 2$ or 1 , and for $7 / 3$, that is 2 or 3 , and so on. Which value is selected will depend on the investor's preference, as long as the consistency requirement is met. The complete comparison matrices for the hypothetical investor are shown in Table 4.6.

Table 4.6: Comparison matrices for hypothetical investor

Aggressive action
\begin{tabular}{|c|c|c|c|}
\hline & A & B & C \\
\hline A & 1 & $1 / 7$ & $1 / 2$ \\
\hline B & 7 & 1 & 3 \\
\hline C & 2 & $1 / 3$ & 1 \\
\hline
\end{tabular}
Nornal action Conservative action

\begin{tabular}{|c|c|c|c|}
\hline & A & B & C \\
\hline A & 1 & $1 / 7$ & 1 \\
\hline B & 7 & 1 & 7 \\
\hline C & 1 & $1 / 7$ & 1 \\
\hline
\end{tabular}

\begin{tabular}{|c|c|c|c|}
\hline & $\mathrm{A}$ & $\mathrm{B}$ & $\mathrm{C}$ \\
\hline $\mathrm{A}$ & 1 & $1 / 3$ & 3 \\
\hline $\mathrm{B}$ & 3 & 1 & 7 \\
\hline $\mathrm{C}$ & $1 / 3$ & $1 / 7$ & 1 \\
\hline
\end{tabular}




\section{Consistency Test and Relative Degree of Preference Calculations}

Similar to matrix $D_{m}$ discussed in Section 3.7, for matrix $D_{3}$ defined in formula (4.6.1), a good estimate of the principal eigenvector (EV) can be described as to multiply the elements in each row and take the $3^{\text {rd }}$ root. The formula is written as:

$$
\begin{aligned}
& E V=\left(b_{A}, b_{B}, b_{C}\right)^{T} \quad(4.6 .4) \\
& \text { where } b_{i}=\left(d_{i A} \times d_{i B} \times d_{i C}\right)^{1 / 3} \quad i=A, B \text {, or } C
\end{aligned}
$$

The normalized principal eigenvector of matrix $D_{3}$ is the vector of priorities (Saaty, 1980, p. 19). It can be obtained by:

$$
\begin{aligned}
& \text { Normalized } E V=\left(b_{A}^{\prime}, b_{B}^{\prime}, b_{C}^{\prime}\right)^{T} \\
& \text { where } b_{i}^{\prime}=b_{i} /\left(b_{A}+b_{B}+b_{C}\right) \quad i=A, B \text {, or } C
\end{aligned}
$$

The eigenvalue of each row of the observed comparison matrix is:

$$
\lambda_{i}=\left(d_{i A} b_{A}+d_{i B} b_{B}+d_{i C} b_{C}\right) / b_{i} \quad i=A, B, \text { or } C
$$

For the observed matrix, the consistency index CI, as defined in formula (3.8.2), is calculated by:

$$
C I=\frac{\lambda_{\max }-3}{2}
$$

From Table 3.5, for the order of matrix $\mathrm{m}=3$, the average random consistency index $\mathrm{RI}=$ 0.52. The consistency ratio $\mathrm{CR}$, as defined in formula (3.8.4), should be no larger than 0.1 for compliance with acceptable consistency. If the consistency of comparison matrix is accepted, then the relative degree of preference between the two reference portfolios, $k$, is estimated by the ratio of the normalized priority values of the two portfolios. This relationship can be expressed as: 


$$
k=\frac{b_{A}^{\prime}}{b_{C}^{\prime}} \quad(4.6 .10)
$$

\subsection{Spreadsheet Model for Calculating the Degree of Preference}

Similar to the spreadsheet model developed in Chapter 3 for calculating objective weights and performing the consistency test, Excel models are designed to calculate the investor's relative degree of preference between reference portfolios and perform the consistency tests. The sample model shown in Figure 4.11. is developed for the situation when the hypothetical investor takes an aggressive action. The formulae and functions used in this model are shown in Figure 4.12.

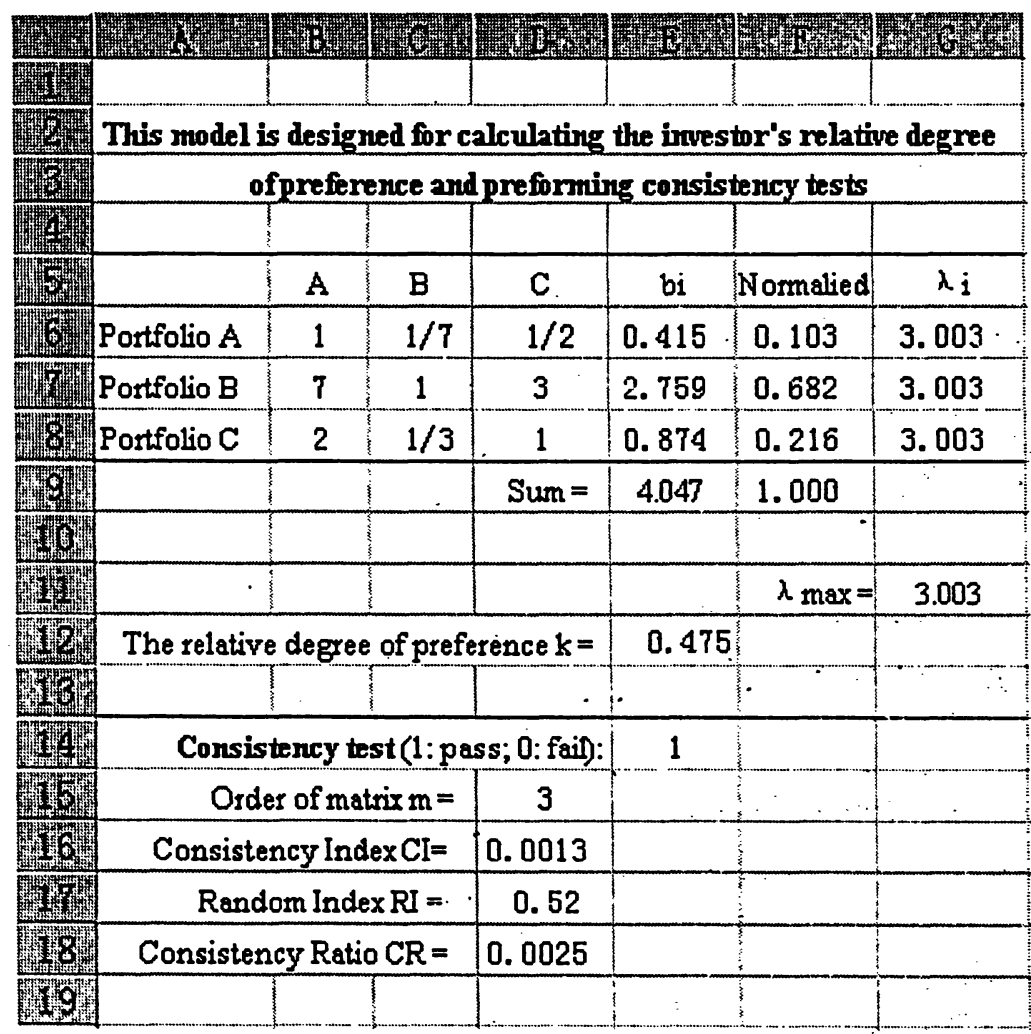

Figure 4.11: Sample Excel model for calculating an investor's relative degree of preference between two reference portfolios 


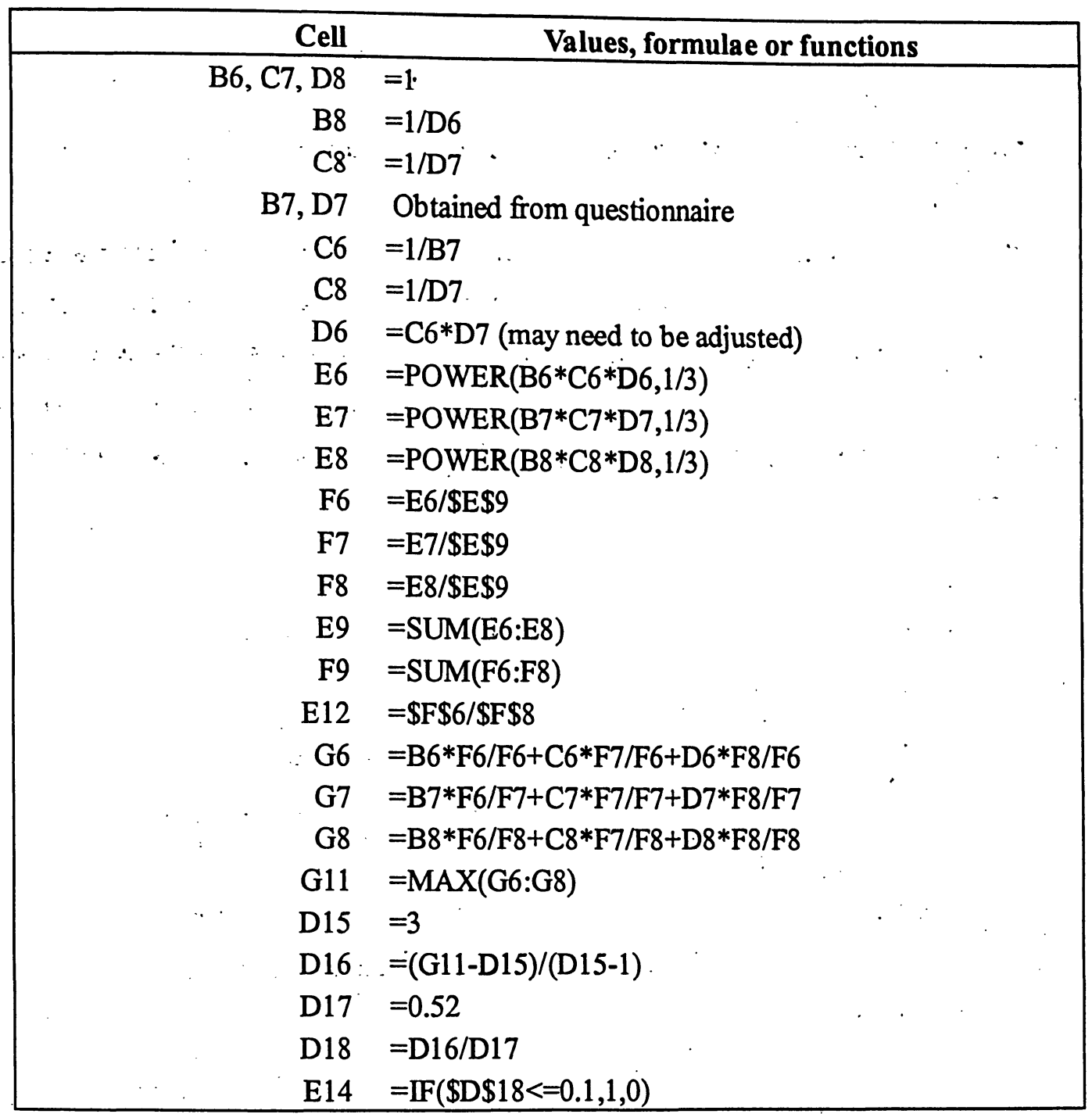

Figure 4.12: Excel model inputs and formulae for Figure 4.11.

In this Excel model, the financial advisor inputs the scale values of the investor's responses to the designed questionnaire shown in Figure 4:10. The number of inputs equals to the number of questions. In fact, only two independent input data are needed to enter into cell B7 and cell D7. Diagonal comparisons are filled with " 1 ". Other values are filled using Excel built-in formulae. The value in cell D6 is obtained using formula D6=C6*D7, which is designed for compliance with initial transitivity requirements. This value can be adjusted according to the investor's preference as long as the consistency requirement is satisfied. The principal eigenvector is shown in cell range E6:E8. The normalized principal 
eigenvector is shown in cell range F6:F8. The consistency test results are shown in cell E14. If the consistency test is passed (CR $<=0.1$ ), the answer is "Yes" (" 1 " in cell E14). If the test is not passed (CR > 0.1), the answer is "No" (" 0 " in cell E14). In this example, the consistency of the matrix is accepted. The relative degree of preference between portfolio $A$ and portfolio $C, k$, is shown in cell $\mathrm{E} 12$, which is obtained by using formula (4.6.10).

Similar models can be designed to calculate the investor's relative degree of preference when he takes normal action or conservative action and to perform the consistency tests. The values of the relative degree of preference for the hypothetical investor, whose comparison matrices are shown in Table 4.6, are summarized in Table 4.7. For different objectives, the same investor may make different responses. Thus the relative degrees of preference between reference portfolios may be different. However, for illustrative purpose, it is assumed that for each of six objectives, the investor makes the similar responses.

Table 4.7: The relative degree of preference for the hypothetical investor

\begin{tabular}{cccc}
\hline & Aggressive action & Normal action & Conservative action \\
\hline $\mathrm{K}$ & 0.475 & 0.630 & 2.759 \\
\hline
\end{tabular}

\subsection{Construction of Local Optimal Portfolios}

After the investor's relative degree of preference between two reference portfolios is obtained, the investor's risk tolerance, $t$, can be calculated using formula (4.2.5). The value of $t$, along with expected returns, standard deviations of asset classes and their correlation coefficients, are the inputs to Model 2.2, which is used to obtain the local optimal portfolio for each objective. Replacing the risk tolerance $t$ with formula (4.2.5), Model 2.2 can be modified. The modified Model 2.2 is shown as Model 4.2. For each local optimization model, the expected returns and standard deviations of two reference portfolios, A and C, can be obtained from Table 4.3, and the investor's relative degree of preference between the two reference portfolios can be obtained from Table 4.7.

\section{Model 4.2:}

Objective:

$$
\text { Maximize } U=E\left(R_{P}\right)-\frac{\sigma_{P}^{2}}{t}
$$


Subject to:

$$
\begin{gathered}
t=\frac{\cdots \sigma_{A}^{2}-k \sigma_{C}^{2}}{E\left(R_{\dddot{A}}\right)-k E\left(R_{C}\right)} \cdots \\
\sigma_{P}^{2}=\sum_{i=1}^{N} x_{i}^{2} \sigma_{i}^{2}+\sum_{\substack { i=1 \\
\begin{subarray}{c}{j \neq 1 \\
j \neq i{ i = 1 \\
\begin{subarray} { c } { j \neq 1 \\
j \neq i } }\end{subarray}}^{N} x_{i} x_{j} \rho_{i j} \sigma_{i} \sigma_{j} \\
E\left(R_{P}\right)=\sum_{i=1}^{N} x_{i} E\left(R_{i}\right) \\
\sum_{i=1}^{N} x_{i}=1 \quad: \quad i=1, \ldots, N \\
x_{i} \geq 0 \quad N \quad
\end{gathered}
$$

where: $\mathrm{U}=$ the utility of portfolio;

$t=$ the investor's risk tolerance;

$k=$ the investor's relative degree of preference between portfolio $\mathrm{A}$ and portfolio $\mathrm{C}$;

$E\left(R_{A}\right)=$ expected return of reference portfolio $A$;

$E\left(R_{C}\right)=$ expected return of reference portfolio $C$;

$\sigma_{\mathrm{A}}=$ standard deviation of return of portfolio $\mathrm{A}$;

$\sigma_{C}=$ standard deviation of return of portfolio $C$;

$E\left(R_{P}\right)=$ expected return of portfolio;

$\sigma_{\mathrm{P}}=$ standard deviation of return of portfolio;

$\mathrm{E}\left(\mathrm{R}_{i}\right)=$ expected return for asset class $i$

$\sigma_{i}=$ standard deviation of return of asset class $i$;

$\rho_{i j}=$ correlation coefficient between asset class $i$ and $j$;

$\mathrm{N}=$ the number of asset classes;

and $\mathrm{x}_{i}=$ proportion of portfolio invested in asset class $i$ :

GAMS programs given in Appendices 1.4, 2.4, 3.4, 4.4, 5.4 and 6.4 are written to solve the quadratic programming model (Model 4.2). The outputs of these programs are characteristics of the local optimal portfolio, including the expected return, standard deviation, percent of each asset class invested, as well as the values of the investor's risk tolerance and utility. 


\section{Chapter 5: Complete Portfolios and Comparisons}

Based on the methods described in Chapter 3 and Chapter 4, a complete portfolio for an individual investor can be obtained. In this chapter, the basic procedure of the proposed multiple-objective asset allocation approach is first reviewed. Then the complete portfolios constructed for the hypothetical investor are presented. A deviation index is suggested to measure the overall inefficiency of complete portfolios from efficient portfolios. Comparisons of the overall efficiency are made among the complete portfolios obtained by the proposed model and those of portfolios obtained by a single-objective optimization method.

\subsection{The Process of Constructing a Complete Portfolio}

In the proposed method, the asset allocation problem for an individual investor is considered as a multiple-objective decision making problem. The general model of the proposed approach for $\mathrm{M}$ objectives and $\mathrm{N}$ asset classes is presented in Figure 3.2. The Analytic Hierarchy Process (AHP) is employed as the framework to address an investor's multiple investment objectives. Traditional portfolio optimization techniques are integrated into the AHP decision hierarchy. In the asset allocation process involving a financial advisor and a client, the client's investment objectives and preferences are reflected through effective communications with the financial advisor. The advisor offers expertise through providing available market opportunities, designing customized questionnaires and helping the client making effective judgments. The result of this asset allocation process is to construct a complete portfolio that is consistent with the investor's investment goals while considering the portfolio's overall efficiency. The basic procedure of constructing such a complete portfolio based on our proposed method is summarized as follows:

Step 1: The financial advisor gathers information from the investor. Questionnaires such as Figure 3.3 are used. The investor makes responses to the questionnaire provided by the financial advisor. The investor's investment objectives and general risk profile are obtained in this step.

Step 2: The financial advisor designs custonized questionnaires such as Figure 3.5 to 
assess the priority of the investor's: multiple investment objectives. 'The investor's responses to the questionnaires are tested for consistency and the relative preferences are evaluated using the AHP techniques. Spreadsheet models such as Figure 3.6 can be used to perform these tasks. The relative importance of the investor's objectives is obtained in this step:

Step 3: The financial advisor transforms the investor's investment objectives into return requirements that can be used as objective functions of appropriate optimization models. The data from capital market research results should be available in this step. For mean-variance optimizations, the data of asset classes' expected return, standard deviation and correlations in terms of each objective are needed. Using these data, the efficient frontier in terms of each objective is obtained. In this step, the financial advisor provides the "market opportunities" to the investor.

Step 4: The financial advisor generates candidate reference portfolios with the aid of computer programs. The investor selects his own reference portfolios from the candidates. Questionnaires such as Figure 4.5 and Figure 4.9 are used. Reference portfolios that reflect the investor's risk attitude and personal preferences are obtained in this step.

Step 5: The investor make responses to questionnaires such as Figure 4.10 to express his preference between his desired portfolio and reference portfolios in terms of each objective. The responses are tested for consistency and the investor's relative degree of preference between reference portfolios is evaluated using the AHP technique. Spreadsheet models such as Figure 4.11 are used to perform these tasks. The investor's risk tolerance is estimated in this step.

Step 6: The financial advisor constructs each local optimal portfolio in terms of each investment objective of the investor. Quadratic programming models such as Model 4.2 are used to combine the market opportunities with the investor's preferences. The local optimal portfolios associated with the investor's objectives are obtained in this step.

Step 7: The financial advisor constructs the complete portfolio by combining the local optimal portfolios according to the relative importance of their associated objectives. The complete portfolio, a mix of different asset classes, is obtained in this step. 


\subsection{Complete Portfolios for the Hypothetical Investor}

In Chapter 3, the relative importance of the six investment objectives of the hypothetical investor is obtained, as shown in Table 3.6 and the left sides of Tables 5.1, 5.2 and 5.3. In Chapter 4, the investor's risk tolerance is incorporated into the associated optimization model to determine the local optimal portfolio for each objective. The local optimal portfolios for the hypothetical investor when he takes aggressive, normal and conservative actions are displayed in the right sides of Tables 5.1, 5.2, and 5.3 respectively. The items for each portfolio include the relative weight, expected return, standard deviation, and the fraction of each assets making up the portfolio. The complete portfolios are obtained by combining the weighted local optimal portfolios according to their relative importance. The proportion of a complete portfolio invested in each individual asset class is obtained using formula (3.2.3). For the hypothetical investor when he takes aggressive, normal and conservative actions, the resulting complete portfolios are displayed at the bottom lines of Tables 5.1, 5.2, and 5.3, respectively.

As it is shown, the complete portfolio for the aggressive action is composed of $28.6 \%$ large-cap stocks, $26.6 \%$ small-cap stocks, $14.8 \%$ long-term corporate bonds, $0.9 \%$ Treasury bills, and $29.1 \%$ real estate. The complete portfolio for the normal action is composed of $28.2 \%$ large-cap stocks, $16.4 \%$ small-cap stocks, $17.7 \%$ long-term corporate bonds, $4.0 \%$ Treasury bills, and $33.7 \%$ real estate. The complete portfolio for the conservation action is composed of $23.2 \%$ large-cap stocks, $8.1 \%$ small-cap stocks, $28.3 \%$ long-term corporate bonds, $0.1 \%$ long-term government bonds, $7.5 \%$ Treasury bills, and $32.8 \%$ real estate. Real estate makes up one of the major components in each case because it performs superior in terms of most of the objectives except the income and liquidity objectives. The investor's low liquidity requirement also contributes to the results. The results also indicate that the complete portfolio tends to smooth the extreme large and extreme small weights of individual assets in local optimal portfolios. Therefore, it can be expected that the complete portfolio is less sensitive to input errors from individual local portfolios. 
Table 5:1: Local optimal and complete portfolios when the investor takes an aggressive action.

\begin{tabular}{|c|c|c|c|c|c|c|c|c|c|}
\hline \multirow{2}{*}{\multicolumn{2}{|c|}{$\begin{array}{l}\text { Objective } \\
\& \text { Weight }\end{array}$}} & \multirow{2}{*}{$\begin{array}{c}E(R) \\
(\%)\end{array}$} & \multirow{2}{*}{$\begin{array}{c}\sigma \\
(\%)\end{array}$} & \multicolumn{6}{|c|}{ Fraction of assets making up the portfolio } \\
\hline & & & & LaStock & SmStock & LCBond & LGBond & T-Bill & RealEst \\
\hline TNR & 0.245 & 11.61 & 16.44 & 0.337 & 0.249 & 0.000 & 0.000 & 0.000 & 0.414 \\
\hline $\mathrm{CA}$ & 0.051 & 9.66 & 19.98 & 0.073 & 0.666 & 0.000 & 0.000 & 0.000 & 0.261 \\
\hline CRI & 0.088 & 5.90 & 3.50 & 0.000 & 0.000 & 1.000 & 0.000 & 0.000 & 0.000 \\
\hline ATR & 0.426 & 8.81 & 13.33 & 0.304 & 0.333 & 0.000 & 0.000 & 0.000 & 0.363 \\
\hline PP & 0.058 & 6.87 & 12.75 & 0.262 & 0.173 & 0.178 & 0.000 & 0.000 & 0.387 \\
\hline $\mathrm{LD}$ : & 0.132 & 8.92 & 12.66 & 0.415 & 0.146 & 0.373 & 0.000 & 0.066 & 0.000 \\
\hline \multicolumn{4}{|c|}{ Complete portfolio } & 0.286 & 0.266 & 0.148 & 0.000 & 0.009 & 0.291 \\
\hline
\end{tabular}

Table 5.2: Local optimal and complete portfolios when the investor takes a normal action.

\begin{tabular}{|c|c|c|c|c|c|c|c|c|c|}
\hline \multirow{2}{*}{\multicolumn{2}{|c|}{$\begin{array}{l}\text { Objective } \\
\text { \& Weight }\end{array}$}} & \multirow{2}{*}{$\begin{array}{c}\mathrm{E}(\mathrm{R}) \\
(\%)\end{array}$} & \multirow{2}{*}{$\begin{array}{c}\sigma \\
(\%)\end{array}$} & \multicolumn{6}{|c|}{ Fraction of assets making up the portfolio } \\
\hline & & & & LaStock & SinStock & LCBond & LGBond & T-Bill & RealEst \\
\hline TNR & 0.245 & 10.12 & 12.80 & 0.308 & 0.149 & 0.150 & 0.000 & 0.000 & 0.393 \\
\hline $\mathrm{CA}$ & 0.051 & 7.94 & 16.01 & 0.189 & 0.447 & 0.000 & 0.000 & 0.000 & 0.364 \\
\hline CRI & 0.088 & 5.90 & 3.50 & 0.000 & 0.000 & 1.000 & 0.000 & 0.000 & 0.000 \\
\hline ATR & 0.426 & 7.73 & 10.52 & 0.336 & 0.193 & 0.000 & 0.000 & 0.000 & 0.471 \\
\hline PP & 0.058 & 6.36 & 11.59 & 0.248 & 0.139 & 0.236 & 0.000 & 0.000 & 0.377 \\
\hline $\mathrm{LD}$ & 0.132 & 7.49 & 9.28 & 0.300 & 0.107 & 0.292 & 0.000 & 0.301 & 0.000 \\
\hline \multicolumn{4}{|c|}{ Complete portfolio } & 0.282 & 0.164 & 0.177 & 0.000 & 0.040 & 0.337 \\
\hline
\end{tabular}

Table 5.3: Local optimal and complete portfolios when the investor takes a conservative action

\begin{tabular}{cccccccccc}
\hline Objective & $\mathrm{E}(\mathrm{R})$ & $\sigma$ & \multicolumn{5}{c}{ Fraction of assets making up the portfolio } \\
\cline { 5 - 11 } \& Weight & $(\%)$ & $(\%)$ & LaStock & SmStock & LCBond & LGBond & T-Bill & RealEst \\
\hline TNR & 0.245 & 8.52 & 9.39 & 0.236 & 0.070 & 0.356 & 0.000 & 0.000 & 0.338 \\
CA $^{-1}$ & 0.051 & 5.99 & 11.99 & 0.257 & 0.250 & 0.000 & 0.000 & 0.090 & 0.403 \\
CRI & 0.088 & 5.90 & 3.50 & 0.000 & 0.000 & 1.000 & 0.000 & 0.000 & 0.000 \\
ATR & 0.426 & 6.37 & 7.51 & 0.290 & 0.085 & 0.148 & 0.000 & 0.000 & 0.477 \\
PP & 0.058 & 5.65 & 10.11 & 0.230 & 0.093 & 0.295 & 0.022 & 0.000 & 0.360 \\
LD & 0.132 & 6.08 & 6.14 & 0.187 & 0.069 & 0.212 & 0.000 & 0.532 & 0.000 \\
\hline \multicolumn{3}{c}{ Complete portfolio } & 0.232 & 0.081 & 0.283 & 0.001 & 0.075 & 0.328 \\
\hline
\end{tabular}

The performance, i.e., expected return and standard deviation, of the complete portfolio in terms of each objective can be obtained by using following equations:

$$
\begin{gathered}
E\left(R_{C p}\right)=\sum_{i=1}^{N} x_{i} E\left(R_{i P}\right) \\
\sigma_{C P}^{2}=\sum_{i=1}^{N} x_{i}^{2} \sigma_{i P}^{2}+\sum_{i=1}^{N} \sum_{\substack{j=1 \\
j \neq i}}^{N} x_{i} x_{j} \sigma_{i P} \sigma_{j P} \rho_{i j}
\end{gathered}
$$


where

$\mathrm{E}\left(\mathrm{R}_{\mathrm{Cp}_{\mathrm{p}}}\right)=$ expected return of complete portfolio in terms of objective $p$;

$\sigma_{\mathrm{C}_{\mathrm{p}}}=$ standard deviation of return of the complete portfolio in terms of objective $p$;

$\mathrm{E}\left(\mathrm{R}_{\mathrm{iP}}\right)=$ expected return for asset class $i$ in terms of objective $p$;

$\sigma_{\mathrm{iP}}=$ standard deviation of return of asset class $i$ in terms of objective $p$;

$\rho_{\mathrm{ij}}=$ correlation coefficient between asset class $i$ and $j$;

$\mathrm{N}=$ the number of asset classes;

and $\mathrm{x}_{\mathrm{i}}=$ proportion of the complete portfolio invested in asset class $i$.

GAMS programs are written to perform these calculations, as shown in Appendices 1.5, 2.5, 3.5, 4.5, 5.5, and 6.5. The results are summarized in Table 5.4.

Table 5.4: The performances of a complete portfolio in terms of each objective

\begin{tabular}{cccccccc}
\hline & & \multicolumn{2}{c}{ Aggressive action } & \multicolumn{2}{c}{ Normal action } & \multicolumn{3}{c}{ Conservative action } \\
\cline { 3 - 8 } Objective & Weight & $\begin{array}{c}\mathrm{E}(\mathrm{R}) \\
(\%)\end{array}$ & $\begin{array}{c}\sigma \\
(\%)\end{array}$ & $\begin{array}{c}\mathrm{E}(\mathrm{R}) \\
(\%)\end{array}$ & $\begin{array}{c}\sigma \\
(\%)\end{array}$ & $\begin{array}{c}\mathrm{E}(\mathrm{R}) \\
(\%)\end{array}$ & $\begin{array}{c}\sigma \\
(\%)\end{array}$ \\
\hline TNR & 0.245 & 11.20 & 15.64 & 9.94 & 12.53 & 8.48 & 9.38 \\
$\mathrm{CA}$ & 0.051 & 6.07 & 12.49 & 4.82 & 10.02 & 3.31 & 7.39 \\
$\mathrm{CRI}$ & 0.088 & 5.12 & 3.21 & 5.12 & 2.57 & 5.18 & 2.10 \\
$\mathrm{ATR}$ & 0.426 & 7.91 & 11.34 & 6.89 & 8.87 & 5.71 & 6.36 \\
$\mathrm{PP}$ & 0.058 & 7.93 & 15.56 & 6.72 & 12.54 & 5.33 & 9.56 \\
LD & 0.132 & 7.71 & 12.57 & 6.55 & 9.98 & 5.48 & 7.30 \\
\hline \multicolumn{2}{l}{ Weighted return } & 8.35 & & 7.32 & & 6.17 & \\
\hline
\end{tabular}

The complete portfolio obtained may not be optimal in terms of each individual objective. The associated efficient portfolios for the complete portfolios in terms of each objective are summarized in Table 5.5. They are minimum variance portfolios with the same expected returns as the complete portfolio in terms of each objective. They are obtained by solving Model 4.1 with given expected returns. GAMS programs are written to perform these tasks, as given in Appendices 1.6, 2.6, 3.6, 4.6, 5.6, and 6.6. For example, the expected return of the normal action portfolio in terms of the total nominal return objective is $9.94 \%$ (see Table 5.4). With expected return of $9.94 \%$, the standard deviation of the efficient portfolio in terms of the total nominal objective is $12.39 \%$ (denoted as $\sigma^{*}$ in Table 5.5), which is slightly less than that of the complete portfolio, $12.53 \%$ (see Table 5.4). Note that an efficient portfolio that is "efficient" in all dimensions may not actually exist at all. Therefore, the efficient portfolios in Table 5.5 are the hypothetical combination of six portfolios; each of them is efficient for its associated objective only. The weighted returns, 
shown at the bottom line of Table 5.4 and Table 5.5, are the weighted average returns for six objectives. They can be used to evaluate the overall aggressiveness of a portfolio in terms of overall investment objectives.

Table 5.5: Associated efficient portfolios of complete portfolios

\begin{tabular}{cccccccc}
\hline & & \multicolumn{2}{c}{$\begin{array}{c}\text { Aggressive action } \\
\text { Efficient portfolios }\end{array}$} & \multicolumn{2}{c}{$\begin{array}{c}\text { Normal action } \\
\text { Efficient portfolios }\end{array}$} & \multicolumn{2}{c}{$\begin{array}{c}\text { Conservative action } \\
\text { Ebjecient portfolios }\end{array}$} \\
\cline { 3 - 9 } & Weight & $\mathrm{E}(\mathrm{R})$ & $\sigma^{*}$ & $\mathrm{E}(\mathrm{R})$ & $\sigma^{*}$ & $\mathrm{E}(\mathrm{R})$ & $\sigma^{*}$ \\
$(\%)$ & $(\%)$ & $(\%)$ & $(\%)$ & $(\%)$ & $(\%)$ \\
\hline TNR & 0.245 & 11.20 & 15.40 & 9.94 & 12.39 & 8.48 & 9.31 \\
$\mathrm{WA}$ & 0.051 & 6.07 & 12.15 & 4.82 & 9.71 & 3.31 & 6.77 \\
CRI & 0.088 & 5.12 & 1.84 & 5.12 & 1.84 & 5.18 & 1.87 \\
ATR & 0.426 & 7.91 & 10.97 & 6.89 & 8.61 & 5.71 & 6.21 \\
PP & 0.058 & 7.93 & 15.37 & 6.72 & 12.41 & 5.33 & 9.51 \\
LD & 0.132 & 7.71 & 9.80 & 6.55 & 7.15 & 5.48 & 4.92 \\
\hline Weighted return & 8.35 & & 7.32 &. & 6.17 & $:$ \\
\hline
\end{tabular}

\subsection{Measurement of Portfolio Efficiency}

A deviation index, $D I$, is used to measure the overall inefficiency of a complete portfolio. $D I$ is defined as the sum of weighted relative deviations of portfolio standard deviations from efficient portfolios in terms of all objectives. The formula of deviation index is written as:

$$
D I=\sum_{j=1}^{M} w_{j} \frac{\sigma_{j}-\sigma_{j}^{*}}{\sigma_{j}^{*}} \times 100 \%
$$

where $D I=$ deviation index of the complete portfolio;

$\mathrm{w}_{j}=$ weight of objective $j$;

$\sigma_{j}=$ standard deviation of return of the complete portfolio in terms of objective $j$;

$\sigma_{j}^{*}=$ standard deviation of return of the efficient portfolio, with the same expected return as the complete portfolio in terms of objective $j$;

$M=$ the number of objectives.

The efficiency of the complete portfolio, E, can be written as:

$$
E=100 \%-D I \text {. (5.3.2) }
$$

Using formulae (5.3.1) and (5.3.2), the deviation index and efficiency for each of the three 
complete portfolios can be calculated. The values of $\sigma_{j}$ and $\sigma_{j}^{*}$ are taken from Tables 5.4 and 5.5, respectively. The results are shown in Table 5.6.

Table 5.6: Deviation index and efficiency of complete portfolios

\begin{tabular}{cccc}
\hline & $\begin{array}{c}\text { Aggressive action } \\
\text { portfolio }\end{array}$ & $\begin{array}{c}\text { Normal action } \\
\text { portfolio }\end{array}$ & $\begin{array}{c}\text { Conservative action } \\
\text { portfolio. }\end{array}$ \\
\hline Weighted return & $8.35 \%$ & $7.32 \%$ & $.6 .17 \%$ \\
Deviation index & $12.3 \%$ & $10.5 \%$ & $9.2 \%$ \\
Efficiency & $87.7 \%$ & $89.5 \%$ & $.90 .8 \%$ \\
\hline
\end{tabular}

Table 5.6 indicates that, for the hypothetical asset allocation example, when a portfolio becomes more aggressive, it has larger weighted deviation from efficient portfolios, and the efficiency of the portfolio decreases.

\subsection{Comparison with Single Objective Optimization}

The proposed multiple objective asset allocation method considers the multiple dimensions of an investor's investment objectives and preferences, some of them are conflicting. Tradeoffs and priorities of these objectives are considered. Therefore, although the suitability of a portfolio is largely subjective, a portfolio constructed using our proposed method can be considered more suitable than the one obtained by using a single objective optimization method, which only considers the returns and risks according to one objective.

As stated in Section 2.4, an optimal portfolio in terms of one objective may not also be optimal in terms of other objectives. Here the overall efficiency of the complete portfolios constructed using the proposed method is compared with those obtained using single-objective optimization. For the hypothetical investor, the weight of the after-tax return (ATR) objective dominates other objectives. Therefore three local optimal portfolios in terms of the after-tax return objective are used for comparisons. These three portfolios are obtained when the hypothetical investor takes aggressive, normal and conservative actions, respectively. The proportions of these three portfolios invested in each asset class are shown in Tables 5.1, 5.2, and 5.3 respectively, under the after-tax return objective. For example, from Table 5.2, when the hypothetical investor takes a normal action, the local optimal portfolio for the after-tax return objective consists of $33.6 \%$ large-cap stocks, 
$19.3 \%$ small-cap stocks and $47.1 \%$ real estates. The performances of this portfolio in terms of each of the six objectives can be obtained by using formulae (5.2.1) and (5.2.2). Revised GAMS programs shown in Appendices 1.5, 2.5, 3.5, 4.5, 5.5 and 6.5 are used to perform the calculations, replacing the set of parameters $x$ (i) with $\{0.336 ; 0: 193,0 ; 0,0,0.471\}$. For the aggressive and conservative actions, the sets of parameters $x(i)$ are replaced. with $\{0.304,0.333,0,0,0,0.363\}$ and $\{0.290,0.085,0.148,0,0,0.477\}$, taken from Tables 5.1 and 5.3 for after-tax return objective respectively. The results are summarized in Table 5.7. Their associated efficient portfolios are summarized in Table 5.8, which are obtained by solving Model 4.1 for each given expected return in terms of each objective. GAMS programs given in Appendices 1.6, 2.6,3.6, 4.6, 5.6 and 6.6 are used to perform these calculations.

Tabie 5.7: Performances of the ATR portfolio in terms of each objective

\begin{tabular}{|c|c|c|c|c|c|c|c|}
\hline \multirow[b]{2}{*}{ Objective } & \multirow[b]{2}{*}{ weight } & \multicolumn{2}{|c|}{ Aggressive action } & \multicolumn{2}{|c|}{ Normal action } & \multicolumn{2}{|c|}{ Conservative action } \\
\hline & & $\begin{array}{c}\mathrm{E}(\mathrm{R}) \\
(\%)\end{array}$ & $\begin{array}{c}\sigma \\
(\%)\end{array}$ & $\begin{array}{c}\mathrm{E}(\mathrm{R}) \\
(\%)\end{array}$ & $\begin{array}{c}\sigma \\
(\%)\end{array}$ & $\begin{array}{c}\mathrm{E}(\mathrm{R}) \\
(\%)\end{array}$ & $\begin{array}{c}\sigma \\
. \%)\end{array}$ \\
\hline TNR & 0.245 & 12.32 & 18.37 & 11.03 & 15.02 & 9.37 & 11.29 \\
\hline $\mathrm{CA}$ & 0.051 & 7.31 & 14.68 & 6.01 & 12.08 & 4.20 & 9.05 \\
\hline CRI & 0.088 & 5.01 & 3.76 & 5.03 & 2.99 & 5.17 & 2.29 \\
\hline ATR & 0.426 & 8.81 & 13.33 & 7.73 & 10.52 & 6.37 & 7.51 \\
\hline PP & $0.05 \overline{8}$ & 8.99 & 18.20 & 7.74 & $14.95^{\circ}$ & 6.16 & 11.34 \\
\hline LD & 0.132 & 8.31 & 14.93 & 6.72 & $11.77^{\circ}$ & 5.35 & $\cdot 8.27$ \\
\hline Weighte & return & 9.20 & & 8.08 & & $6.47^{\circ}$ & \\
\hline
\end{tabular}

Table 5.8: Associated efficient portfolios of the ATR portfolios

\begin{tabular}{ccccccccc}
\hline & & \multicolumn{2}{c}{$\begin{array}{c}\text { Aggressive action } \\
\text { Efficient portfolios }\end{array}$} & \multicolumn{2}{c}{ Normal action } \\
Efficient portfolios & \multicolumn{2}{c}{$\begin{array}{c}\text { Conservative action } \\
\text { Ebjectivent portfolios }\end{array}$} \\
\cline { 3 - 9 } & Weight & $\mathrm{E}(\mathrm{R})$ & $\sigma^{*}$ & $\mathrm{E}(\mathrm{R})$ & $\sigma^{*}$ & $\mathrm{E}(\mathrm{R})$ & $\sigma^{*}$ \\
$(\%)$ & $(\%)$ & $. \%)$ & $(\%)$ & $(\%)$ & $(\%)$ \\
\hline TNR & 0.245 & 12.32 & 18.35 & 11.03 & 14.99 & 9.37 & 11.18 \\
CA & 0.051 & 7.31 & 14.64 & 6.01 & 12.04 & 4.20 & 8.50 \\
CRI & 0.088 & 5.01 & 1.78 & 5.03 & 1.79 & 5.17 & 1.87 \\
ATR & 0.426 & 8.81 & $13.33:$ & 7.73 & 10.52 & 6.37 & 7.51 \\
PP & 0.058 & 8.99 & 18.17 & 7.74 & 14.89 & 6.16 & 11.16 \\
LD & 0.132 & 8.31 & 11.21 & 6.72 & 7.53 & 5.35 & 4.68 \\
\hline \multicolumn{2}{l}{ Weighted return } & 9.20 & & 8.08 & & 6.47 & \\
\hline
\end{tabular}

The deviation index and efficiency of the three porffolios are obtained using formulae (5.3.1) and (5.3.2). The results are shown in Table 5.9. Table 5.9 also indicates that, when a 
portfolio becomes more aggressive, it has a larger weighted deviation from efficient portfolios, and the efficiency of the portfolio decreases. Comparisons between Tables 5.6 and 5.9 indicate in all three cases, the efficiencies of portfolios obtained from our proposed multiple objective method are higher than those of portfolios obtained by using after-tax return objective optimization. The overall efficiency of the complete portfolios in terms of all investment objectives is improved.

Table 5.9: Deviation index and efficiency of the ATR portfolios

\begin{tabular}{cccc}
\hline & $\begin{array}{c}\text { Aggressive action } \\
\text { portfolio }\end{array}$ & $\begin{array}{c}\text { Normal action } \\
\text { portfolio }\end{array}$ & $\begin{array}{c}\text { Conservative action } \\
\text { portfolio }\end{array}$ \\
\hline Weighted return & $9.20 \%$ & $8.08 \%$ & $6.47 \%$ \\
Deviation index & $14.2 \%$ & $13.4 \%$ & $12.6 \%$ \\
Efficiency & $85.8 \%$ & $86.6 \%$ & $87.4 \%$ \\
\hline
\end{tabular}




\section{Chapter 6: Conclusions and Future Work}

\subsection{Summary of Contributions}

The main contributions of this project report are as follows:

(1) An asset allocation model that is consistent with an individual investor's multiple investment objectives and preferences is developed. The concept of the Analytic Hierarchy Process (AHP) is employed to construct the multiple objective decision model, while traditional portfolio optimization techniques are integrated into the hierarchy. Both of the suitability and efficiency of the complete portfolio are considered. In . comparison with single-objective optimization, improved overall efficiency in terms of all objectives is achieved. The complete portfolio constructed is less sensitive to input errors from individual optimization models.

(2) A method to measure an individual investor's risk tolerance is proposed. In this method, risk tolerance is defined as the ratio of added portfolio variances to added portfolio returns, considering the investor's relative degree of preference between two reference portfolios. The investor selects his own reference portfolios from a list of candidates provided by the financial advisor. The relative degree of preference is estimated by the AHP technique. Risk tolerance measured using the proposed method can be incorporated into a quadratic programming model to determine a local optimal portfolio.

(3) An interactive procedure based on the proposed model is suggested to implement the asset allocation process involving a financial advisor and an individual investor. Five sample questionnaires are designed for the following tasks: (i) to gather information about an investor's investment objectives and general risk profile; (ii) to assess an investor's preference over different investment objectives; (iii) to select aggressive and conservative reference portfolios; and (iv) to assess an investor's preference over the reference portfolios.

(4) Computer programs are developed to perform the following tasks: (i) to find the global 
minimum variance portfolios; (ii) to find the maximum return portfolios; and (iii) to generate the lists of aggressive and conservative reference portfolios. 36 sample programs are written for a hypothetical asset allocation example with six investment objectives and six asset classes. These programs can be generalized to solve mean-variance portfolio optimization models with $\mathrm{N}$ numbers of asset classes.

(5) Two sample spreadsheet models based on the AHP technique are designed to perform priority analysis and to perform consistency tests for an investor's responses.

\subsection{Future Work}

By utilizing the AHP method, the proposed method provides a mechanism to integrate portfolio optimization methods into a single asset allocation decision model. However, only classical mean-variance optimization techniques are employed in the hypothetical investment example in this project report. No extra efforts are made to include other alternative portfolio optimization models. Considering only the variance as the portfolio risk surely cannot explain many investors' investment behaviors. One direction of future work is to consider various risk measurements according to an investor's risk attitude. For example, if an investor believes the downside variance, a measure of portfolio variability below the expected return, is a more appropriate portfolio risk measure in terms of current income objective, then mean-semivariance optimization methods can be used to obtain the local optimal portfolio in terms of the current income objective, while other local optimal portfolios may be determined by using other appropriate optimization techniques.

The quadratic programming models adopted in this report are based on the classical Markowitz models, which consider only the budget constraints and nonnegative weights of assets. No additional constraints, such as transaction costs and minimum transaction unit requirements, are considered. Ignoring these constraints is largely based on the consideration that general solution techniques are not available at present for specially structured portfolio problems. However, including these constraints to a model represent more realistic investment situations in real world. Therefore, the proposed model would benefit from the continuing research of portfolio optimization techniques. 
Appendices

\section{Appendix 1}

GAMS Programs Associated with the Total Nominal Return Objective 


\section{Appendix 1.1}

\section{GAMS Program for Finding the Global Minimum Variance Portfolio in terms of the Total Nominal Return Objective}

\section{\$OnText}

This sample GAMS program is written to find the global minimal variance portfolio along efficient frontier in terms of the total nominal return (TNR) objective. The underlying quadratic programming model is Model 4.1.

\section{\$OffText}

Set i assets /LaStock, SmStock,LCBond, LGBond,TBill, RealEst/; alias (i,j);

Scalars $k$ index for loop target initial target return /4.2/;

Parameters return(i) expected nominal total returns of individual assets (\%)

\begin{tabular}{ll|} 
LaStock & 12.1 \\
SmStock & 17.8 \\
LCBond & 5.3 \\
LGBond & 4.7 \\
TBill & 3.6 \\
RealEst & $7.5 \quad /$;
\end{tabular}

Parameters STD(i) standard deviations of TNR of individual assets(\%)

$\begin{array}{lr}\text { LaStock } & 20.9 \\ \text { SmStock } & 35.6 \\ \text { LCBond } & 8.4 \\ \text { LGBond } & 7.5 \\ \text { TBill } & 3.3 \\ \text { RealEst } & 15.0 \quad / \text {; }\end{array}$

Table col(i,j) correlations between assets

$\begin{array}{lcccccc} & \text { LaStock } & \text { SmStock } & \text { LCBond } & \text { LGBond } & \text { TBill } & \text { RealEst } \\ \text { LaStock } & 1.00 & 0.82 & 0.19 & 0.11 & -0.07 & -0.06 \\ \text { SmStock } & 0.82 & 1.00 & 0.08 & -0.01 & -0.08 & 0.06 \\ \text { LCBond } & 0.19 & 0.08 & 1.00 & 0.93 & 0.19 & -0.08 \\ \text { LGBond } & 0.11 & -0.01 & 0.93 & 1.00 & 0.22 & -0.09 \\ \text { TBill } & -0.07 & -0.08 & 0.19 & 0.22 & 1.00 & 0.38 \\ \text { RealEst } & -0.06 & 0.06 & -0.08 & -0.09 & 0.38 & 1.00\end{array}$




\begin{tabular}{|c|c|c|}
\hline Variables & $\begin{array}{l}\mathrm{x}(\mathrm{i}) \\
\text { variance } \\
\text { standey }\end{array}$ & $\begin{array}{l}\text { fraction of portfolio invested in asset } i \\
\text { variance of portfolio } \\
\text { standard deviation of portfolio }\end{array}$ \\
\hline
\end{tabular}

\section{Equations}

$\begin{array}{llll} & \begin{array}{l}\text { fsum } \\ \text { dmean }\end{array} & \begin{array}{l}\text { fractions must add to 1.0. } \\ \text { definition of mean expected return on portfolio }\end{array} \\ & \begin{array}{lll}\text { fvariance } \\ \text { definition of portfolio variance; }\end{array} \\ \text { fsum.. } & \operatorname{sum}(\mathrm{i}, \mathrm{x}(\mathrm{i})) & =\mathrm{e}=1.0 ; \\ \text { dmean.. } & \operatorname{sum}(\mathrm{i}, \mathrm{return}(\mathrm{i}) * \mathrm{x}(\mathrm{i})) & =\mathrm{e}=\text { target; } \\ \text { fvariance.. } & \operatorname{sum}(\mathrm{i}, \mathrm{x}(\mathrm{i}) * \operatorname{sum}(\mathrm{i}, \operatorname{col}(\mathrm{i}, \mathrm{j}) * \operatorname{STD}(\mathrm{i}) * \operatorname{STD}(\mathrm{j}) * \mathrm{x}(\mathrm{j}))) & =\mathrm{e}= & \text { variance; }\end{array}$

Model portfolio / all/;

Solve portfolio using nlp minimizing variance;

$$
\begin{aligned}
& \text { target }=\text { target }-0.1 \text {; } \\
& \text { Scalars v1 ; } \\
& \text { Scalars v2; } \\
& \mathrm{v1}=\text { variance. } 1 \text {; } \\
& \text { * set v1 current variance }
\end{aligned}
$$

Model p1 /fsum, dmean, fvariance/;

Solve p1 using nlp minimizing variance;

$$
\begin{aligned}
& \text { v2 = variance.1; } \\
& \mathrm{k}=1 \text {; } \\
& \text { For }(k=1 \text { to } 6 \text {, } \\
& \text { If }(\mathrm{v} 1>\mathrm{v} 2 \text {, } \\
& \text { target }=\text { target }-0.1 \text {; } \\
& \mathrm{v} 1=\mathrm{v} 2 \text {; }
\end{aligned}
$$

Solve $\mathrm{pl}$ using nlp minimizing variance;

v2 = variance.l;

\section{Else}

target $=$ target +0.1 ;

Solve $p 1$ using nlp minimizing variance;

standev. $1=\operatorname{sqrt}(\mathrm{v} 1)$

Display v1, standev.l, target ;

);

target $=$ target -0.1 ; 


\section{Appendix 1.2}

\section{GAMS Program for Generating the List of Candidate Portfolios in terms of the Total Nominal Return Objective}

\section{\$OnText}

This sample GAMS program is written to generate the candidate conservative reference portfolio list in terms of the total nominal return (TNR) objective. Slightly modified, this program can also be used to generate the aggressive portfolio list. The underlying quadratic programming model is Model 4.1.

\section{\$OffText}

Set $\quad \mathrm{i}$ assets /LaStock, SmStock,LCBond, LGBond,TBill, RealEst/; alias (i,j) Scalar target targeted mean after-tax return on portfolio (\%) /4/;

* If generate aggressive portfolio, the target value can be set as 15 , for example. Scalar $k$ index for loop;

Parameters return(i) expected nominal total returns of individual assets (\%)

$\begin{array}{llr}\text { / LaStock } & 12.1 \\ \text { SmStock } & 17.8 \\ \text { LCBond } & 5.3 \\ \text { LGBond } & 4.7 \\ \text { TBill } & 3.6 \\ \text { RealEst } & 7.5 \quad / \text {; }\end{array}$

Parameters STD(i) standard deviations of TNR of individual assets (\%)

\begin{tabular}{|lr} 
| LaStock & 20.9 \\
SmStock & 35.6 \\
LCBond & 8.4 \\
LGBond & 7.5 \\
TBill & 3.3 \\
RealEst & $15.0 \quad$ /;
\end{tabular}

Table col(i, $\left.i_{2}\right)$ correlations between assets

$\begin{array}{lcccccc} & \text { LaStock } & \text { SmStock } & \text { LCBond } & \text { LGBond } & \text { TBill } & \text { RealEst } \\ \text { LaStock } & 1.00 & 0.82 & 0.19 & 0.11 & -0.07 & -0.06 \\ \text { SmStock } & 0.82 & 1.00 & 0.08 & -0.01 & -0.08 & 0.06 \\ \text { LCBond } & 0.19 & 0.08 & 1.00 & 0.93 & 0.19 & -0.08 \\ \text { LGBond } & 0.11 & -0.01 & 0.93 & 1.00 & 0.22 & -0.09 \\ \text { TBill } & -0.07 & -0.08 & 0.19 & 0.22 & 1.00 & 0.38 \\ \text { RealEst } & -0.06 & 0.06 & -0.08 & -0.09 & 0.38 & 1.00 \\ & & & 80 & & & \end{array}$




$\begin{array}{lll}\text { Variables } & \mathrm{x}(\mathrm{i}) & \text { fraction of portfolio invested in asset } \mathrm{i} \\ \text { variance } & \text { variance of portfolio } \\ \text { standev } & \text { standard deviation of portfolio }\end{array}$

\section{Positive Variable $\mathrm{x}$;}

Equations fsum fractions must add to 1.0

dmean -- definition of expected TNR on portfolio

fvariance definition of portfolio variance;

$\begin{array}{lll}\text { fsum.. } & \operatorname{sum}(\mathrm{i}, \mathrm{x}(\mathrm{i})) & =\mathrm{e}=1.0 ; \\ \text { dmean.. } & \operatorname{sum}(\mathrm{i}, \operatorname{return}(\mathrm{i}) * \mathrm{x}(\mathrm{i})) & =\mathrm{e}=\text { target } ; \\ \text { fvariance.. } & \operatorname{sum}(\mathrm{i}, \mathrm{x}(\mathrm{i}) * \operatorname{sum}(\mathrm{j}, \operatorname{col}(\mathrm{i}, \mathrm{j}) * \operatorname{STD}(\mathrm{i}) * \operatorname{STD}(\mathrm{j}) * \mathrm{x}(\mathrm{j}))) & =\mathrm{e}=\text { variance; }\end{array}$

Model portfolio / all/;

Solve portfolio using nlp minimizing variance;

standev.1 = sqrt(variance.l);

Display target, variance.l, standev.l, x.l;

For $(k=1$ to 5 ,

target $=$ target +0.5 ;

* If used for generating aggressive portfolio list, target = target - step

* In this example, the length of step is set at 0.5

Solve portfolio using nlp minimizing variance;

standev. 1 = sqrt(variance.l);

Display target, variance.l, standev.l, x.l;

); 


\section{Appendix 1.3}

\section{GAMS Program for Finding the Maximum Return Portfolio in terms of the Total Nominal Return Objective}

\section{\$OnText}

This sample GAMS program is written to find the maximum return portfolio along the efficient frontier in terms of the total nominal return (TNR) objective. This portfolio is considered the most aggressive. The underlying quadratic \$OffText programming model is Model 2.1.

Set i assets /LaStock, SmStock,LCBond, LGBond,TBill, RealEst/; alias (i,j);

Scalar $\mathrm{k}$ index for loop;

Scalar TargetSTD target standard deviation of portfolio (\%) /33/;

Parameters return(i) expected nominal total returns of individual assets (\%)

$\begin{array}{llr}\text { / LaStock } & 12.1 \\ \text { SmStock } & 17.8 \\ \text { LCBond } & 5.3 \\ \text { LGBond } & 4.7 \\ \text { TBill } & 3.6 \\ \text { RealEst } & 7.5 \quad / \text {; }\end{array}$

Parameters STD(i) standard deviations of TNR on individual assets (\%)

$\begin{array}{lr}\text { LaStock } & 20.9 \\ \text { SmStock } & 35.6 \\ \text { LCBond } & 8.4 \\ \text { LGBond } & 7.5 \\ \text { TBill } & 3.3 \\ \text { RealEst } & 15.0 \quad \text { /; }\end{array}$

Table col(i,j) correlations between assets

$\begin{array}{lcccccc} & \text { LaStock } & \text { SmStock } & \text { LCBond } & \text { LGBond } & \text { TBill } & \text { RealEst } \\ \text { LaStock } & 1.00 & 0.82 & 0.19 & 0.11 & -0.07 & -0.06 \\ \text { SmStock } & 0.82 & 1.00 & 0.08 & -0.01 & -0.08 & 0.06 \\ \text { LCBond } & 0.19 & 0.08 & 1.00 & 0.93 & 0.19 & -0.08 \\ \text { LGBond } & 0.11 & -0.01 & 0.93 & 1.00 & 0.22 & -0.09 \\ \text { TBill } & -0.07 & -0.08 & 0.19 & 0.22 & 1.00 & -0.38 \\ \text { RealEst } & -0.06 & 0.06 & -0.08 & -0.09 & 0.38 & 1.00\end{array}$


Variables $\quad x(i) \quad$ fraction of portfolio invested in asset $i$

Positive Variable x;

ExReturn expected return on portfolio

Equations

fsum . fractions must add to 1.0

dmean : definition of mean expected TNR on portfolio

fSTD definition of standard deviation of portfolio;

fsum.. $\operatorname{sum}(i, x(i)) \quad=e=1.0 \quad$;

dmean.. $\operatorname{sum}(i, \operatorname{return}(i) * x(i)) \quad=e=$ ExReturn;

fSTD.. $\quad \operatorname{sqrt}(\operatorname{sum}(i, x(i) * \operatorname{sum}(j, \operatorname{col}(i, j) * \operatorname{STD}(i) * \operatorname{STD}(j) * x(j))))=e=$ TargetSTD;

Model portfolio / all /;

Solve portfolio using nlp maximizing ExReturn;

Display ExReturn.1, targetSTD, x.1 ;

* Now change the value of targetSTD

targetSTD $=\operatorname{targetSTD}+0.2$

Scalars R1;

Scalars R2 ;

R1 = ExReturn.1;

Model p1 /fsum, dmean, ISTD/;

Solve p1 using nlp maximizing ExReturn;

R2 = ExReturn.1;

$\mathrm{k}=1$;

For $(\mathrm{k}=1$ to 20 ,

If $(R 1<R 2$,

targetSTD $=$ targetSTD +0.2 ;

$\mathrm{R} 1=\mathrm{R} 2$;

Solve p1 using nlp maximizing ExReturn;

R2 = ExReturn.1;

Else

targetSTD $=$ targetSTD -0.2 ;

Solve p1 using nlp maximizing ExReturn;

Display ExReturn.1, targetSTD, x.1 ;

);

$\operatorname{targetSTD}=$ targetSTD +0.2 ;

); 


\section{Appendix 1.4}

\section{GAMS Program for Obtaining Local Optimal Portfolio in terms of the Total Nominal Return Objective}

\section{\$OnText}

This GAMS program is written to solve the quadratic programming model (Model 4.2) whose objective function is to maximize an investor's utility in terms of the total nominal return (TNR) objective. The outputs are the characteristics of local optimal portfolio, including the expected return, standard deviation, percentage of portfolio invested in each asset class, as well as the values. of the investor's risk tolerance and utility.

\section{SOffText}

Set $\mathrm{i}$ assets /LaStock, SmStock,LCBond, LGBond,TBill, RealEst/; alias (i,j);

Set $m$ reference portfolio $A / \operatorname{ReA}, \operatorname{Var} A /$;

Set 1 reference portfolio $\mathrm{C} / \mathrm{ReC}$, $\operatorname{VarC}$;

Scalar $t$ the investor's risk tolerance;

Scalar $k$ the relative degree of preference between portfolios A and C / $0.630 /$;

* For aggressive action, $\mathrm{k}=0.475$

* For normal action, $\mathrm{k}=0.630$

$* \quad$ For conservative action, $\mathrm{k}=2.759$

Parameters refA(m) expected return and variance of reference portfolio $A$

$$
\begin{array}{cc}
/ \operatorname{ReA} & 4.00 \\
\text { VarA } & 9.99 \quad / ;
\end{array}
$$

Parameters refC(l) expected return and variance of reference portfolio $\mathrm{C}$

/ ReC $\quad 13.00$

VarC 411.68 /;

$\mathrm{t}=\left(\operatorname{refA}\left({ }^{\prime} \operatorname{Var} \mathrm{A}^{\prime}\right)-\mathrm{k} * \operatorname{refC}\left({ }^{\prime} \operatorname{Var} C^{\prime}\right)\right) /\left(\operatorname{refA}\left({ }^{\prime} \operatorname{ReA}{ }^{\prime}\right)-\mathrm{k} * \operatorname{refC}\left({ }^{\prime} \operatorname{ReC} C^{\prime}\right)\right)$;

* Definition of an investor's risk tolerance

Parameters return(i) expected nominal total returns of individual assets (\%).

$\begin{array}{lr}\text { LaStock } & 12.1 \\ \text { SmStock } & 17.8 \\ \text { LCBond } & 5.3 \\ \text { LGBond } & 4.7 \\ \text { TBill } & 3.6 \\ \text { RealEst } & 7.5 \quad / \text {; }\end{array}$


Parameters STD(i) standard deviations of TNR of individual assets (\%)

$\begin{array}{lr}\text { LaStock } & 20.9 \\ \text { SmStock } & 35.6 \\ \text { LCBond } & 8.4 \\ \text { LGBond } & 7.5 \\ \text { TBill } & 3.3 \\ \text { RealEst } & 15.0 \quad / \text {; }\end{array}$

Table col(i,j) correlations between assets

$\begin{array}{lccccccc} & \text { LaStock } & \text { SmStock } & \text { LCBond } & \text { LGBond } & \text { TBill } & \text { RealEst } & \\ \text { LaStock } & 1.00 & 0.82 & 0.19 & 0.11 & -0.07 & -0.06 & \\ \text { SmStock } & 0.82 & 1.00 & 0.081 & -0.01 & -0.08 & 0.06 & \\ \text { LCBond } & 0.19 & 0.08 & 1.00 & 0.93 & 0.19 & -0.08 & \\ \text { LGBond } & 0.11 & -0.01 & 0.93 & 1.00 & 0.22 & -0.09 & \\ \text { TBill } & -0.07 & -0.08 & 0.19 & 0.22 & 1.00 & 0.38 & \\ \text { RealEst } & -0.06 & 0.06 & -0.08 & -0.09 & 0.38 & 1.00 & ;\end{array}$
Variables $\quad x(i) . \quad$ fraction of portfolio invested in asset $i$
returnEx expected return of portfolio variance definition of potfolio variance standev standard deviation of portfolio utility definition of utility;

Positive Variable x;

Equations

$\begin{array}{ll}\text { fsum } & \text { fractions must add to } 1.0 \\ \text { freturnEx } & \text { definition of portfolio return } \\ \text { fvariance } & \text { definition of potfolio variance } \\ \text { dutil } & \text { definition of utility; }\end{array}$
fsum.. $\quad \operatorname{sum}(\mathrm{i}, \mathrm{x}(\mathrm{i}))$
freturnEx.. $\operatorname{sum}(i, \operatorname{return}(i) * x(i))$
$=\mathrm{e}=1.0$;
fvariance..
$\operatorname{sum}(\mathrm{i}, \mathrm{x}(\mathrm{i}) * \operatorname{sum}(\mathrm{j}, \operatorname{col}(\mathrm{i}, \mathrm{j}) * \operatorname{STD}(\mathrm{i}) * \operatorname{STD}(\mathrm{j}) * \mathrm{x}(\mathrm{j}))$
$=\mathrm{e}=$ returnEx;
dutil.. returnEx - variance/t
$=e=$ variance;
$=e=$ utility ;

Model portfolio / all /;

Solve portfolio using nlp maximizing utility;

standev.1 = sqrt(variance. 1$) \quad$;

Display t, utility.l, returnEx.1, standev.l, x.1; 


\section{Appendix 1.5}

\section{GAMS Program for Finding Performance of the Complete Portfolio in terms of the Total Nominal Return Objective}

\section{SOnText}

This GAMS program is written to obtain the performance information (expected return and standard deviation) of the complete portfolio in terms of the total nominal return (TNR) objective.

\section{\$OffText}

Set $i$ assets /LaStock, ȘmStock,LCBond, LGBond,TBill, RealEst/; alias (i,j)

Scalar ReturnC Excepted return of complete portfolio in terms of TNR objective $\operatorname{VarC} \quad$ Variance of complete portfolio in terms of TNR objective StdC Standard deviation;

Parameters $\mathrm{x}(\mathrm{i})$ fraction of assets making up the complete portfolio

$\begin{array}{lll}\text { / LaStock } & 0.282 \\ \text { SmStock } & 0.164 \\ \text { LCBond } & 0.177 \\ \text { LGBond } & 0.000 \\ \text { T⿱Bill } & 0.040 \\ \text { RealEst } & 0.337 \quad / \text {; }\end{array}$

*These data are for normal action;

* For aggressive action, LaStock $=0.286$, SmStock $=0.266, \mathrm{LCBond}=0.148$,

* LGBond $=0.000$, TBill $=0.009$, RealEst $=0.291$.

* For conservative action, LaStock $=0.232$, SmStock $=0.081$, LCBond $=0.283$,

* LGBond $=0.001$, TBill $=0.075$, RealEst $=0.328$.

Parameters return(i) expected nominal total returns of individual assets (\%)

\begin{tabular}{|lr} 
/ LaStock & 12.1 \\
SmStock & 17.8 \\
LCBond & 5.3 \\
LGBond & 4.7 \\
TBill & 3.6 \\
RealEst & $7.5 \quad$ /;
\end{tabular}


Parameters STD(i) standard deviations of TNR of individual assets (\%)

$\begin{array}{llr}\text { LaStock } & 20.9 \\ \text { SmStock } & 35.6 \\ \text { LCBond } & 8.4 \\ \text { LGBond } & 7.5 \\ \text { TBill } & 3.3 \\ \text { RealEst } & 15.0 \quad / ;\end{array}$

Table col(i,j) correlations between assets

$\begin{array}{lccccccc} & \text { LaStock } & \text { SmStock } & \text { LCBond } & \text { LGBond } & \text { TBill } & \text { RealEst } & \\ \text { LaStock } & 1.00 & 0.82 & 0.19 & 0.11 & -0.07 & -0.06 & \\ \text { SmStock } & 0.82 & 1.00 & 0.08 & -0.01 & -0.08 & 0.06 & \\ \text { LCBond } & 0.19 & 0.08 & 1.00 & 0.93 & 0.19 & -0.08 & \\ \text { LGBond } & 0.11 & -0.01 & 0.93 & 1.00 & 0.22 & -0.09 & \\ \text { TBill } & -0.07 & -0.08 & 0.19 & 0.22 & 1.00 & 0.38 & \\ \text { RealEst } & -0.06 & 0.06 & -0.08 & -0.09 & 0.38 & 1.00 & ;\end{array}$

$$
\begin{aligned}
& \operatorname{returnC}=\operatorname{sum}(\mathrm{i}, \operatorname{return}(\mathrm{i}) * \mathrm{x}(\mathrm{i})) ; \\
& \operatorname{varC}=\operatorname{sum}(\mathrm{i}, \mathrm{x}(\mathrm{i}) * \operatorname{sum}(\mathrm{j}, \operatorname{col}(\mathrm{i}, \mathrm{j}) * \operatorname{STD}(\mathrm{i}) * \operatorname{STD}(\mathrm{j}) * \mathrm{x}(\mathrm{j}))) \\
& \operatorname{stdC}=\operatorname{sqrt}(\operatorname{var} \mathrm{C})
\end{aligned}
$$

Display ReturnC, StdC; 


\section{Appendix 1.6}

\section{GAMS Program for Obtaining the. Optimal Portfolio for a Given Target Return in terms of the Total Nominal Return Objective}

\section{SOnText}

This GAMS program is written to solve the quadratic programming model (Model 4.1) whose objective is to minimize a portfolio's variance in terms of the nominal total return (TNR) objective. The input data are the specified expected target returns. The outputs are the characteristics of optimal portfolio, including the expected return, standard deviation, and the percentage of portfolio invested in each asset class.

\section{\$OffText}

Set $i$ assets /LaStock, SmStock,LCBond, LGBond,TBill, RealEst/; alias (i,j) Scalar target target mean total nominal return (TNR) of portfolio (\%) / 13/;

Parameters return(i) expected nominal total returns of individual assets (\%)

$\begin{array}{lr}\text { / LaStock } & 12.1 \\ \text { SmStock } & 17.8 \\ \text { LCBond } & 5.3 \\ \text { LGBond } & 4.7 \\ \text { TBill } & 3.6 \\ \text { RealEst } & 7.5 \quad / \text {; }\end{array}$

Parameters STD(i) standard deviations of TNR on individual assets (\%)

$\begin{array}{llr}\text { LaStock } & 20.9 \\ \text { SmStock } & 35.6 \\ \text { LCBond } & 8.4 \\ \text { LGBond } & 7.5 \\ \text { TBill } & 3.3 \\ \text { RealEst } & 15.0 \quad / \text {; }\end{array}$

Table col( $\mathrm{i}, \mathrm{j})$ correlations between assets

$\begin{array}{lcccccc} & \text { LaStock } & \text { SmStock } & \text { LCBond } & \text { LGBond } & \text { TBill } & \text { RealEst } \\ \text { LaStock } & 1.00 & 0.82 & 0.19 & 0.11 & -0.07 & -0.06 \\ \text { SmStock } & 0.82 & 1.00 & 0.08 & -0.01 & -0.08 & 0.06 \\ \text { LCBond } & 0.19 & 0.08 & 1.00 & 0.93 & 0.19 & -0.08 \\ \text { LGBond } & 0.11 & -0.01 & 0.93 & 1.00 & 0.22 & -0.09 \\ \text { TBill } & -0.07 & -0.08 & 0.19 & 0.22 & 1.00 & 0.38 \\ \text { RealEst } & -0.06 & 0.06 & -0.08 & -0.09 & 0.38 & 1.00\end{array}$



Variables $\quad x(i) \quad$ fraction of portfolio invested in asset $i$ variance variance of expected TRN on portfolio standev. standard deviation of portfolio ...

Positive Variable x;

Equations fsum fractions must add to 1.0

dmean . . definition of expected TNR on portfolio

fvariance definition of portfolio variance;

fsum.. $\quad \operatorname{sum}(\mathbf{i}, \mathbf{x}(\mathbf{i}))$

dmean.. $\quad \operatorname{sum}(i, \operatorname{return}(i) * x(i))$

fvariance.. $\quad \operatorname{sum}(i, x(i) * \operatorname{sum}(j, \operatorname{col}(i, j) * \operatorname{STD}(i) * \operatorname{STD}(j) * x(j)))$ $=\mathrm{e}=1.0$;

$=\mathrm{e}=$ target;

$=\mathrm{e}=$ variance;

Model portfolio / all/;

Solve portfolio using nlp minimizing variance;

standev.l = sqrt(variance.l);

Display target, variance.1, standev.1, x.l; 
Appendix 2

GAMS Programs Associated with the Capital Appreciation Objective 


\section{Appendix 2.1}

\section{GAMS Program for Finding the Global Minimum Variance Portfolio in terms of the Capital Appreciation Objective}

\section{\$OnText}

This GAMS program is written to find the global minimum variance portfolio along efficient frontier in terms of the capital appreciation (CA) objective. The underlying quadratic programming model is Model 4.1.

\section{\$OffText}

Set $\quad \mathrm{i}$ assets /LaStock, SmStock,LCBond, LGBond,TBill, RealEst/; alias (i,j);

Scalars $k$ index for loop target initial target return /0.2/;

Parameters return(i) expected capital appreciations of individual assets (\%)

$\begin{array}{llr}\text { LaStock } & 7.3 \\ \text { SmStock } & 12.8 \\ \text { LCBond } & -0.6 \\ \text { LGBond } & -0.4 \\ \text { TBill } & -0.1 \\ \text { RealEst } & 2.3 \quad / \text {; }\end{array}$

Parameters STD(i) standard deviations of expected CA of individual assets (\%)

$\begin{array}{lr}\text { LaStock } & 17.6 \\ \text { SmStock } & 27.8 \\ \text { LCBond } & 4.9 \\ \text { LGBond } & 4.6 \\ \text { TBill } & 1.0 \\ \text { RealEst } & 11.7 \quad / \text {; }\end{array}$

Table col(i,j) correlations between assets

$\begin{array}{lcccccc} & \text { LaStock } & \text { SmStock } & \text { LCBond } & \text { LGBond } & \text { TBill } & \text { RealEst } \\ \text { LaStock } & 1.00 & 0.82 & 0.19 & 0.11 & -0.07 & -0.06 \\ \text { SmStock } & 0.82 & 1.00 & 0.08 & -0.01 & -0.08 & 0.06 \\ \text { LCBond } & 0.19 & 0.08 & 1.00 & 0.93 & 0.19 & -0.08 \\ \text { LGBond } & 0.11 & -0.01 & 0.93 & 1.00 & 0.22 & -0.09 \\ \text { TBill } & -0.07 & -0.08 & 0.19 & 0.22 & 1.00 & 0.38 \\ \text { RealEst } & -0.06 & 0.06 & -0.08 & -0.09 & 0.38 & 1.00\end{array}$


Variables $\quad x(i) \quad$ fraction of portfolio invested in asset $i$

variance variance of portfolio

standev standard deviation of portfolio

Positive Variable $\mathrm{x}$;

Equations

fsum fractions must add to 1.0

dmean definition of mean expected return on portfolio

fvariance definition of portfolio variance;

fsum.. $\operatorname{sum}(\mathrm{i}, \mathrm{x}(\mathrm{i})) \quad=\mathrm{e}=1.0$;

dmean.. $\operatorname{sum}(\mathbf{i}, \operatorname{return}(\mathbf{i}) * x(\mathbf{i})) \quad=e=$ target;

fvariance.. $\quad \operatorname{sum}(i, x(i) * \operatorname{sum}(j, \operatorname{col}(i, j) * \operatorname{STD}(i) * \operatorname{STD}(j) * x(j))) \quad=e=$ variance;

Model portfolio / all/;

Solve portfolio using nlp minimizing variance;

target $=$ target -0.1 ;

Scalars v1;

Scalars v2 ;

$\mathrm{v} 1=$ variance. $1 ;$

* set vl current variance

Model pl /fsum, dmean, fvariance/;

Solve $\mathrm{pl}$ using nlp minimizing variance;

v2 = variance.l;

$\mathrm{k}=1$;

For $(k=1$ to 6 ,

If $(\mathrm{v} 1>\mathrm{v} 2$,

target $=$ target -0.1 ;

$\mathrm{v} 1=\mathrm{v} 2 ;$

Solve $\mathrm{p} 1$ using nlp minimizing variance;

v2 = variance.l;

Else

target $=$ target +0.1 ;

Solve $\mathrm{p} 1$ using nlp minimizing variance;

standev. 1 = sqrt(v1)

Display v1, standev.l, target ;

) ;

target $=$ target -0.1 ;

); 


\section{GAMS Program for Generating the List of Candidate Portfolio list in terms of the Capital Appreciation Objective}

\section{\$OnText}

This GAMS program is written to generate the candidate conservative reference portfolio list in terms of the capital appreciation (CA) objective. Slightly modified, this program can also be used to generate the aggressive portfolio list.

\$OffText

Set i : assets /LaStock, SmStock,LCBond, LGBond,TBill, RealEst/; alias (i,j)

Scalar target targeted mean after-tax return on portfolio (\%) $12.8 /$;

* If generate aggressive portfolio, the target can be set as 12, for example.

Scalar $\mathrm{k}$ index for loop;

Parameters return(i) expected capital appreciations of individual assets (\%)

\begin{tabular}{|lr} 
/ LaStock & 7.3 \\
SmStock & 12.8 \\
LCBond & -0.6 \\
LGBond & -0.4 \\
TBill & -0.1 \\
RealEst & $2.3 \quad /$;
\end{tabular}

Parameters STD(i) standard deviations of individual assets (\%)

\begin{tabular}{|lr} 
/ LaStock & 17.6 \\
SmStock & 27.8 \\
LCBond & 4.9 \\
LGBond & 4.6 \\
TBill & 1.0 \\
RealEst & $11.7 \quad$ /;
\end{tabular}

Table col(i,j) correlations between assets

$\begin{array}{lcccccc} & \text { LaStock } & \text { SmStock } & \text { LCBond } & \text { LGBond } & \text { TBill } & \text { RealEst } \\ \text { LaStock } & 1.00 & 0.82 & 0.19 & 0.11 & -0.07 & -0.06 \\ \text { SmStock } & 0.82 & 1.00 & 0.08 & -0.01 & -0.08 & 0.06 \\ \text { LCBond } & 0.19 & 0.08 & 1.00 & 0.93 & 0.19 & -0.08 \\ \text { LGBond } & 0.11 & -0.01 & 0.93 & 1.00 & 0.22 & -0.09 \\ \text { TBill } & -0.07 & -0.08 & 0.19 & 0.22 & 1.00 & 0.38 \\ \text { RealEst } & -0.06 & 0.06 & -0.08 & -0.09 & 0.38 & 1.00 \\ & & & 93 & & & \end{array}$;


Variables . $x(i) \quad$ fraction of portfolio invested in asset $i$

variance variance of portfolio

standev standard deviation of portfolio

Positive Variable $x$;

Equations fsum fractions must add to 1.0

dmean definition of expected CA on portfolio

fvariance definition of portfolio variance;

$\begin{array}{lll}\text { fsum.. } & \operatorname{sum}(i, x(i)) & =e=1.0 ; \\ \text { dmean.. } & \operatorname{sum}(i, \operatorname{return}(i) * x(i)) & =e=\text { target } ; \\ \text { fvariance.. } & \operatorname{sum}(i, x(i) * \operatorname{sum}(j, \operatorname{col}(i, j) * \operatorname{STD}(i) * \operatorname{STD}(j) * x(j))) & =e=\text { variance; }\end{array}$

Model portfolio / all /;

Solve portfolio using nlp minimizing variance;

standev.1 = sqrt(variance.l);

Display target, variance.1, standev.l, x.l;

For $(k=1$ to 5 ,

target $=$ target +0.5 ;

* If used for generating aggressive portfolio list, target = target - step

* In this example, the length of step is set at 0.5

Solve portfolio using nlp minimizing variance;

standev.l = sqrt(variance.l) ;

Display target, variance.1, standev.1, x.l;

); 


\section{GAMS Program for Finding the Maximum Return Portfolio in terms of the Capital Appreciation Objective}

\section{\$OnText}

This GAMS program is written to find the maximum return portfolio along the efficient frontier in terms of the capital appreciation (CA) objective. This portfolio is considered the most aggressive. The underlying quadratic programming model is Model 2.1.

\section{\$OffText}

Set $\quad \mathrm{i}$ assets /LaStock, SmStock,LCBönd, LGBond,TBill; RealEst/; alias (i,j); Scalar k index for loop

Scalar TargetSTD target standard deviation on portfolio (\%) /26/;

Parameters return(i) expected capital appreciations of individual assets (\%)

$\begin{array}{lr}\text { LaStock } & 7.3 \\ \text { SmStock } & 12.8 \\ \text { LCBond } & -0.6 \\ \text { LGBond } & -0.4 \\ \text { TBill } & -0.1 \\ \text { RealEst } & 2.3 \quad / \text {; }\end{array}$

Parameters STD(i) standard deviations of CA of individual assets (\%)

$\begin{array}{lr}\text { LaStock } & 17.6 \\ \text { SmStock } & 27.8 \\ \text { LCBond } & 4.9 \\ \text { LGBond } & 4.6 \\ \text { TBill } & 1.0 \\ \text { RealEst } & 11.7 \quad \text { /; }\end{array}$

Table col(i,j) correlations between assets

$\begin{array}{lcccccc} & \text { LaStock } & \text { SmStock } & \text { LCBond } & \text { LGBond } & \text { TBill } & \text { RealEst } \\ \text { LaStock } & 1.00 & 0.82 & 0.19 & 0.11 & -0.07 & -0.06 \\ \text { SmStock } & 0.82 & 1.00 & 0.08 & -0.01 & -0.08 & 0.06 \\ \text { LCBond } & 0.19 & 0.08 & 1.00 & 0.93 & 0.19 & -0.08 \\ \text { LGBond } & 0.11 & -0.01- & 0.93 & 1.00 & 0.22 & -0.09 \\ \text { TBill } & -0.07 & -0.08 & 0.19 & 0.22 & 1.00 & 0.38 \\ \text { RealEst } & -0.06 & 0.06 & -0.08 & -0.09 & 0.38 & 1.00\end{array}$;


Variables $\quad x(i) \quad$ fraction of portfolio invested in asset $i$

ExReturn expected return on portfolio

Positive Variable x;

Equations

fsum fractions must add to 1.0

dmean definition of expected CA on portfolio

FSTD definition of standard deviation of portfolio ;

fsum.. $\quad \operatorname{sum}(\mathrm{i}, \mathrm{x}(\mathrm{i}))$

$=\mathrm{e}=1.0 ;$

dmean.. $\operatorname{sum}(\mathrm{i}, \operatorname{return}(\mathrm{i}) * x(\mathrm{i})) \quad=\mathrm{e}=$ ExReturn;

fSTD.. $\quad \operatorname{sqrt}(\operatorname{sum}(i, x(i) * \operatorname{sum}(j, \operatorname{col}(i, j) * \operatorname{STD}(i) * \operatorname{STD}(j) * x(j))))=e=\operatorname{TargetSTD}$;

Model portfolio / all /;

Solve portfolio using nlp maximizing ExReturn; ।

Display ExReturn.l, targetSTD, x.1 ;

* Now change the value of targetSTD

targetSTD $=$ targetSTD +0.2

Scalars R1 ;

Scalars R2;

$\mathrm{R} 1=$ ExReturn.l;

Model p1 /fsum, dmean, fSTD/;

Solve p1 using nlp maximizing ExReturn;

$\mathrm{R} 2=$ ExReturn.l;
$\mathrm{k}=1 ;$

For $(k=1$ to 20 ,

If $(R 1<R 2$,

targetSTD $=$ targetSTD +0.2 ;

$\mathrm{R} 1=\mathrm{R} 2$;

Solve p1 using nlp maximizing ExReturn;

R2 = ExReturn.l;

Else

targetSTD $=$ targetSTD -0.2 ;

Solve p1 using nlp maximizing ExReturn;

Display ExReturn.1, targetSTD, x.1 ;

);

targetSTD $=$ targetSTD +0.2 ;

) 


\section{Appendix 2.4}

\section{GAMS Program for Obtaining Local Optimal Portfolio in terms of the Capital Appreciation Objective}

\section{\$OnText}

This GAMS program is written to solve the quadratic programming model (Model 4.2) whose objective function is to maximize an investor's utility in terms of the capital appreciation (CA) objective. The outputs are the characteristics of local optimal portfolio, including the expected return, standard deviation, percentage of portfolio invested in each asset class, as.well as the values of the investor's risk tolerance and utility.

\section{\$OffText}

Set i assets /LaStock, SmStock,LCBond, LGBond,TBill, RealEst/; alias (i,j);

Set $\mathrm{m}$ reference portfolio $\mathrm{A} / \mathrm{ReA}$, VarA/;

Set 1 reference portfolio $\mathrm{C} / \mathrm{ReC}, \operatorname{Var} \mathrm{C} /$;

Scalar $t$ the investor's risk tolerance;

Scalar $\mathrm{k}$ the relative degree of preference between portfolios A and C / 0.630/;

* For aggressive action, $\mathrm{k}=0.475$

* For normal action, $\mathrm{k}=0.630$

* For conservative action, $\mathrm{k}=2.759$

Parameters refA(m) expected return and variance of reference portfolio $A$

$/ \operatorname{ReA} \quad 3.00$

VarA $38.01 /$;

Parameters refC(l) expected return and variance of reference portfolio $\mathrm{C}$

I $\mathrm{ReC} \quad 10.00$

$\operatorname{VarC} \quad 431.81 /$;

$\mathrm{t}=\left(\operatorname{refA}\left({ }^{\prime} \operatorname{Var} \mathrm{A}^{\prime}\right)-\mathrm{k} * \operatorname{refC}\left({ }^{\prime} \operatorname{Var} C^{\prime}\right)\right) /\left(\operatorname{ref}\left({ }^{\prime} \operatorname{Re} A^{\prime}\right)-\mathrm{k} * \operatorname{refC}\left({ }^{\prime} \operatorname{ReC} '\right)\right) ;$

* definition of an investor's risk tolerance

Parameters return(i) expected capital appreciations of individual assets (\%)

$\begin{array}{ll}\text { / LaStock } & 7.3 \\ \text { SmStock } & 12.8 \\ \text { LCBond } & -0.6 \\ \text { LGBond } & -0.4 \\ \text { TBill } & -0.1 \\ \text { RealEst } & 2.3 \quad / \text {; }\end{array}$


Parameters STD(i) standard deviations of individual assets (\%)

$\begin{array}{lr}\text { LaStock } & 17.6 \\ \text { SmStock } & 27.8 \\ \text { LCBond } & 4.9 \\ \text { LGBond } & 4.6 \\ \text { TBill } & 1.0 \\ \text { RealEst } & 11.7 \quad / \text {; }\end{array}$

Table col(i,j) correlations between assets

$\begin{array}{lcccccc} & \text { LaStock } & \text { SmStock } & \text { LCBond } & \text { LGBond } & \text { TBill } & \text { RealEst } \\ \text { LaStock } & 1.00 & 0.82 & 0.19 & 0.11 & -0.07 & -0.06 \\ \text { SmStock } & 0.82 & 1.00 & 0.08 & -0.01 & -0.08 & 0.06 \\ \text { LCBond } & 0.19 & 0.08 & 1.00 & 0.93 & 0.19 & -0.08 \\ \text { LGBond } & 0.11 & -0.01 & 0.93 & 1.00 & 0.22 & -0.09 \\ \text { TBill } & -0.07 & -0.08 & 0.19 & 0.22 & 1.00 & 0.38 \\ \text { RealEst } & -0.06 & 0.06 & -0.08 & -0.09 & 0.38 & 1.00\end{array}$

Variables $\quad x(i) \quad$ fraction of portfolio invested in asset $i$

returnEx expected return of portfolio

variance definition of portfolio variance

standev standard deviation of portfolio

utility definition of the utility ;

Positive Variable $\mathrm{x}$;

Equations

fsum fractions must add to 1.0

freturnEx definition of portfolio return

fvariance definition of portfolio variance

dutil definition of utility;

fsum.. $\quad \operatorname{sum}(i, x(i))$

freturnEx.. $\operatorname{sum}(i, \operatorname{return}(i) * x(i))$

fvariance.. $\operatorname{sum}(i, x(i) * \operatorname{sum}(j, \operatorname{col}(i, j) * \operatorname{STD}(i) * \operatorname{STD}(j) * x(j)))$

dutil.. returnEx - variance/t $=\mathrm{e}=1.0$;

$=\mathrm{e}=$ returnEx ;

$=\mathrm{e}=$ variance;

$=\mathrm{e}=$ utility ;

Model portfolio / all/;

Solve portfolio using nlp maximizing utility;

standev.l = sqrt(variance. 1 ) ;

Display t, utility.l, returnEx.l, standev.l, x.1; 


\section{Appendix 2.5}

\section{GAMS Program for Finding Performance of the Complete Portfolio in terms of the Capital Appreciation Objective}

\section{SOnText}

This GAMS program is written to obtain the performance information (expected return and standard deviation) of the complete portfolio in terms of the capital appreciation (CA) objective.

\$OffText

Set $i$ assets /LaStock, SmStock,LCBond, LGBond,TBill, RealEst/; alias (i,j)

Scalar ReturnC Excepted return of complete portfolio in terms of CA objective VarC Variance of complete portfolio in terms of CA objective

StdC Standard deviation;

Parameters $\mathbf{x}(\mathbf{i})$ fraction of assets making up the complete portfolio

$\begin{array}{lll}\text { LaStock } & 0.282 \\ \text { SmStock } & 0.164 \\ \text { LCBond } & 0.177 \\ \text { LGBond } & 0.000 \\ \text { TBill } & 0.040 \\ \text { RealEst } & 0.337 \quad / \text {; }\end{array}$

*These data are for normal action;

$*$ For aggressive action, LaStock $=0.286$, SmStock $=0.266, \mathrm{LCB}$ ond $=0.148$,

$* \quad$ LGBond $=0.000, \mathrm{TBill}=0.009$, RealEst $=0.291$.

$*$ For conservative action, LaStock $=0.232$, SmStock $=0.081, \mathrm{LCB}$ - $\mathrm{Sd}=0.283$,

$* \quad$ LGBond $=0.001$, TBill $=0.075$, RealEst $=0.328$.

Parameters return(i) expected capital appreciations of individual assets (\%)

$\begin{array}{lr}\text { LaStock } & 7.3 \\ \text { SmStock } & 12.8 \\ \text { LCBond } & -0.6 \\ \text { LGBond } & -0.4 \\ \text { TBill } & -0.1 \\ \text { RealEst } & 2.3 \quad / ;\end{array}$


Parameters STD(i) standard deviations of of CA of individual asset(\%)

$\begin{array}{lr}\text { / LaStock } & 17.6 \\ \text { SmStock } & -27.8 \\ \text { LCBond } & 4.9 \\ \text { LGBond } & 4.6 \\ \text { TBill } & 1.0 \\ \text { RealEst } & .11 .7 \quad / \text {; }\end{array}$

Table col(i,j) correlations between assets

$\begin{array}{llcccccc} & \text { LaStock } & \text { SmStock } & \text { LCBond } & \text { LGBond } & \text { TBill } & \text { RealEst } \\ & \text { LaStock } & 1.00 & 0.82 & 0.19 & 0.11 & -0.07 & -0.06 \\ & \text { SmStock } & 0.82 & 1.00 & 0.08 & -0.01 & -0.08 & 0.06 \\ \text { LCBond } & 0.19 & 0.08 & 1.00 & 0.93 & 0.19 & -0.08 \\ \text { LGBond } & 0.11 & -0.01 & 0.93 & 1.00 & 0.22 & -0.09 \\ \text { TBill } & -0.07 & -0.08 & 0.19 & 0.22 & 1.00 & 0.38 \\ \text { RealEst } & -0.06 & 0.06 & -0.08 & -0.09 & 0.38 & 1.00\end{array}$

Display ReturnC, StdC; 


\section{Appendix 2.6}

\section{GAMS Program for Obtaining the Optimal Portfolio for a Given Target Return in terms of the Capital Appreciation Objective}

\section{SOnText}

This GAMS program is written to solve the quadratic programming model (Model 4.1) whose objective function is to minimize a portfolio's variance in terms of the capital appreciation (CA) objective. The input data are the specified expected target returns. The outputs are the characteristics of optimal portfolio, including the expected return, standard deviation, percentage of portfolio invested in each asset class.

\section{SOffText}

Set $i$ assets /LaStock, SmStock,LCBond, LGBond,TBill, RealEst/; alias (i,j)

Scalar target target mean capital appreciation (CA) of portfolio (\%)/10/;

Parameters return(i) expected capital appreciations of individual assets (\%)

$\begin{array}{lr}\text { LaStock } & 7.3 \\ \text { SmStock } & 12.8 \\ \text { LCBond } & -0.6 \\ \text { LGBond } & -0.4 \\ \text { TBill } & -0.1 \\ \text { RealEst } & 2.3 \quad / \text {; }\end{array}$

Parameters STD(i) standard deviations of individual assets (\%)

$\begin{array}{lr}\text { / LaStock } & 17.6 \\ \text { SmStock } & 27.8 \\ \text { LCBond } & 4.9 \\ \text { LGBond } & 4.6 \\ \text { TBill } & 1.0 \\ \text { RealEst } & 11.7 \quad / \text {; }\end{array}$

Table col( $i, j)$ correlations between assets

$\begin{array}{lcccccc} & \text { LaStock } & \text { SmStock } & \text { LCBond } & \text { LGBond } & \text { TBill } & \text { RealEst } \\ \text { LaStock } & 1.00 & 0.82 & 0.19 & 0.11 & -0.07 & -0.06 \\ \text { SmStock } & 0.82 & 1.00 & 0.08 & -0.01 & -0.08 & 0.06 \\ \text { LCBond } & 0.19 & 0.08 & 1.00 & 0.93 & 0.19 & -0.08 \\ \text { LGBond } & 0.11 & -0.01 & 0.93 & 1.00 & 0.22 & -0.09 \\ \text { TBill } & -0.07 & -0.08 & 0.19 & 0.22 & 1.00 & 0.38 \\ \text { RealEst } & -0.06 & 0.06 & -0.08 & -0.09 & 0.38 & 1.00\end{array}$


Variables . $\quad x(i) \quad$ fraction of portfolio invested in asset $i$

variance variance of portfolio

standev standard deviation of portfolio

Positive Variable x;

Equations fsum fractions must add to 1.0

dmean definition of mean expected CA on portfolio

fvariance definition of portfolio variance;

fsum.. $\quad \operatorname{sum}(i, x(i))$

dmean.. $\quad \operatorname{sum}(i, \operatorname{return}(i) * x(i))$

fvariance..

$\operatorname{sum}(i, x(i) * \operatorname{sum}(j, c o l(i, j)$ $=\mathrm{e}=1.0 ;$

$=\mathrm{e}=$ target;

$=\mathrm{e}=$ variance;

Model portfolio / all /;

Solve portfolio using nlp minimizing variance;

standev.1 = sqrt(variance.1) ;

Display target, variance.l, standev.l, x.l; 
Appendix 3

GAMS Programs Associated with the Current Income Objective . 


\section{Appendix 3.1}

\section{GAMS Program for Finding the Global Minimum Variance Portfolio in terms of the Current Income Objective}

SOnText

This GAMS program is written to find the global minimum variance portfolio along efficient frontier in terms of the current income (CRI) objective. The underlying quadratic programming model is Model 4.1.

SOffText

Set i assets /LaStock, SmStock,LCBond, LGBond,TBill, RealEst/; alias (i,j);

Scalars $k$ index for loop target 15.0/;

Parameters return(i) expected current incomes of individual assets (\%)

$\begin{array}{ll}\text { / LaStock } & 4.8 \\ \text { SmStock } & 5.0 \\ \text { LCBond } & 5.9 \\ \text { LGBond } & 4.9 \\ \text { TBill } & 3.7 \\ \text { RealEst } & 5.2 \quad / ;\end{array}$

Parameters STD(i) standard deviations of CRI of individual assets (\%)

$\begin{array}{lll}\text { LaStock } & 3.3 \\ \text { SmStock } & 8.0 \\ \text { LCBond } & 3.5 \\ \text { LGBond } & 2.9 \\ \text { TBill } & 2.4 \\ \text { RealEst } & 3.3 \quad / \text {; }\end{array}$

Table col(i,j) correlations between assets

$\begin{array}{lcccccc} & \text { LaStock } & \text { SmStock } & \text { LCBond } & \text { LGBond } & \text { TBill } & \text { RealEst } \\ \text { LaStock } & 1.00 & 0.82 & 0.19 & 0.11 & -0.07 & -0.06 \\ \text { SmStock } & 0.82 & 1.00 & 0.08 & -0.01 & -0.08 & 0.06 \\ \text { LCBond } & 0.19 & 0.08 & 1.00 & 0.93 & 0.19 & -0.08 \\ \text { LGBond } & 0.11 & -0.01 & 0.93 & 1.00 & 0.22 & -0.09 \\ \text { TBill } & -0.07 & -0.08 & 0.19 & 0.22 & 1.00 & 0.38 \\ \text { RealEst } & -0.06 & 0.06 & -0.08 & -0.09 & 0.38 & 1.00\end{array}$




$\begin{array}{lll}\text { Variables } & \begin{array}{c}\mathrm{x}(\mathrm{i}) \\ \text { variance } \\ \text { standev. }\end{array} & \begin{array}{l}\text { fraction of portfolio invested in asset } \mathrm{i} \\ \text { variance of portfolio } \\ \text { standard deviation of portfolio }\end{array} \\ \text { Positive Variable } \mathrm{x} ; & \ldots\end{array}$

\section{Equations}

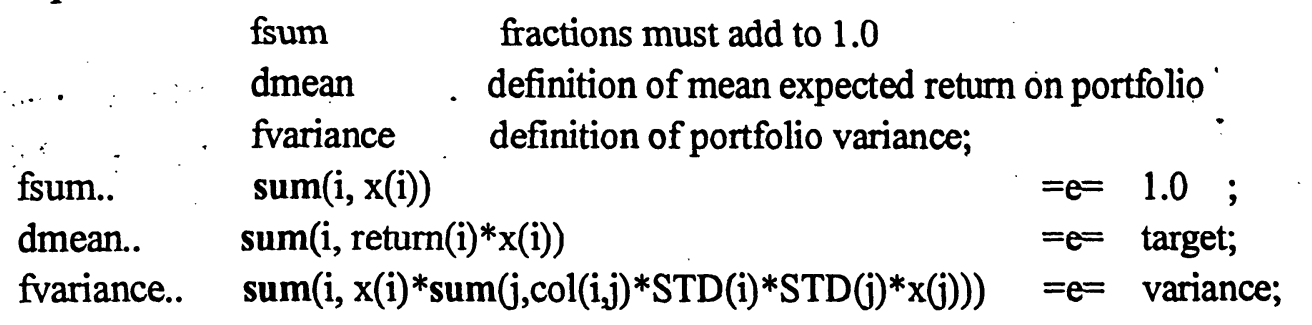

Model portfolio / all / ;

Solve portfolio using nlp minimizing variance;

$$
\begin{aligned}
& \text { target }=\text { target }-0.1 ; \\
& \text { Scalars v1; } \\
& \text { Scalars v2; } \\
& \text { v1 = variance.1; } \\
& * \quad \text { set v1 current variance }
\end{aligned}
$$

Model p1 /fsum, dmean, fvariance/;

Solve pl using nlp minimizing variance;

$$
\begin{aligned}
& \text { v2 = variance.l; } \\
& \mathrm{k}=1 \text {; } \\
& \text { For }(\mathrm{k}=1 \text { to } 6 \text {, } \\
& \text { If }(\mathrm{v} 1>\mathrm{v} 2 \text {, } \\
& \text { target }=\text { target }-0.1 \text {; } \\
& \mathrm{v} 1=\mathrm{v} 2 \text {; }
\end{aligned}
$$

Solve $\mathrm{p} 1$ using nIp minimizing variance;

v2 = variance.l;

Else

target $₹$ target +0.1 ;

Solve $\mathrm{p} 1$ using nlp minimizing variance;

standev. $1=\operatorname{sqrt}(\mathrm{v} 1)$

Display v1, standev.1, target ;

target $=$ target -0.1 ;

) 


\section{Appendix 3.2}

\section{GAMS Program for Generating the List of Candidate Portfolios in terms of the Current Income Objective}

\section{\$OnText}

This GAMS program is written to generate the candidate conservative reference portfolio list in terms of the current income (CRI) objective: Slightly modified, this program can also be used to generate the aggressive portfolio list.

\$OffText

Set $\quad \mathrm{i}$ assets /LaStock, SmStock,LCBond, LGBond,TBill, RealEst/; alias (i,j) Scalar target targeted mean income on portfolio (\%) /4.5/;

* If generate aggressive portfolio, the target value can be set as 5.9, for example . Scalar $\mathrm{k}$ index for loop;

Parameters return(i) expected current incomes of individual assets (\%)

$\begin{array}{ll}\text { LaStock } & 4.8 \\ \text { SmStock } & 5.0 \\ \text { LCBond } & 5.9 \\ \text { LGBond } & 4.9 \\ \text { TBill } & 3.7 \\ \text { RealEst } & 5.2 \quad / \text {; }\end{array}$

Parameters STD(i) standard deviations of CRI of individual assets (\%)

$\begin{array}{lll}\text { LaStock } & 3.3 \\ \text { SmStock } & 8.0 \\ \text { LCBond } & 3.5 \\ \text { LGBond } & 2.9 \\ \text { TBill } & 2.4 \\ \text { RealEst } & 3.3 \cdot / \text {; }\end{array}$

Table col(i,j) correlations between assets

$\begin{array}{lcccccc} & \text { LaStock } & \text { SmStock } & \text { LCBond } & \text { LGBond } & \text { TBill } & \text { RealEst } \\ \text { LaStock } & 1.00 & 0.82 & 0.19 & 0.11 & -0.07 & -0.06 \\ \text { SmStock } & 0.82 & 1.00 & 0.08 & -0.01 & -0.08 & 0.06 \\ \text { LCBond } & 0.19 & 0.08 & 1.00 & 0.93 & 0.19 & -0.08 \\ \text { LGBond } & 0.11 & -0.01 & 0.93 & 1.00 & 0.22 & -0.09 \\ \text { TBill } & -0.07 & -0.08 & 0.19 & 0.22 & 1.00 & 0.38 \\ \text { RealEst } & -0.06 & 0.06 & -0.08 & -0.09 & 0.38 & 1.00\end{array}$



Variables $\quad x(i) \quad$ fraction of portfolio invested in asset $i$ variance variance of portfolio standev ... standard deviation of portfolio

Positive Variable $x$;

Equations f fum : fractions must add to 1.0

$\begin{array}{ll}\text { - dmean } & \text { definition of expected income on portfolio } \\ \text { fvariance } & \text { definition of portfolio variance; }\end{array}$

fsum.. $\quad \operatorname{sum}(i, x(i)) \quad=e=1.0$;

dmean.. $\quad \operatorname{sum}(\mathrm{i}, \operatorname{return}(\mathrm{i}) * \mathrm{x}(\mathrm{i})) \quad:=\mathrm{e}=$ target ;

fvariance.. $\quad \operatorname{sum}(i, x(i) * \operatorname{sum}(j, \operatorname{col}(i, j) * \operatorname{STD}(i) * S T D(j) * x(j)))=e=$ variance;

Model portfolio / all / ;

Solve portfolio using nlp minimizing variance;

standev.l = sqrt(variance. 1$)$;

Display target, variance.l, standev.l, x.l;

For $(\mathrm{k}=1$ to 5 ,

$$
\text { target }=\text { target }+0.5
$$

* If used for generating aggressive portfolio list, target = target - step

* In this example, the length of step is set at 0.5

Solve portfolio using nlp minimizing variance;

standev.1 = sqrt(variance.l) ;

Display target, variance.1, standev.l, x.l;

) ; 


\section{Appendix 3.3}

\section{GAMS Program for. Finding the Maximum Return Portfolio in terms of the Current Income Objective}

\$OnText

This GAMS program is written to find the maximum return portfolio along the efficient frontier in terms of the current income (CRI) objective. This portfolio is considered the most aggressive. The underlying quadratic programming model is Model 2.1.

\section{\$OffText}

Set i assets /LaStock, SmStock,LCBond, LGBond,TBill, RealEst/; alias (i,j);

Scalar $\mathrm{k}$ index for loop

Scalar TargetSTD target standard deviation of portfolio (\%) / 2.9/;

Parameters return(i) expected current incomes of individual assets (\%)

$\begin{array}{ll}\text { LaStock } & 4.8 \\ \text { SmStock } & 5.0 \\ \text { LCBond } & 5.9 \\ \text { LGBond } & 4.9 \\ \text { TBill } & 3.7 \\ \text { RealEst } & 5.2 \quad / \text {; }\end{array}$

Parameters STD(i) standard deviations of CRI of individual assets (\%)

$\begin{array}{lll}\text { / LaStock } & 3.3 \\ \text { SmStock } & 8.0 \\ \text { LCBond } & 3.5 \\ \text { LGBond } & 2.9 \\ \text { TBill } & 2.4 \\ \text { RealEst } & 3.3 \quad / ;\end{array}$

Table col(i,j) correlations between assets

$\begin{array}{lcccccc} & \text { LaStock } & \text { SmStock } & \text { LCBond } & \text { LGBond } & \text { TBill } & \text { RealEst } \\ \text { LaStock } & 1.00 & 0.82 & 0.19 & 0.11 & -0.07 & -0.06 \\ \text { SmStock } & 0.82 & 1.00 & 0.08 & -0.01 & -0.08 & 0.06 \\ \text { LCBond } & 0.19 & 0.08 & 1.00 & 0.93 & 0.19 & -0.08 \\ \text { LGBond } & 0.11 & -0.01 & 0.93 & 1.00 & 0.22 & -0.09 \\ \text { TBill } & -0.07 & -0.08 & 0.19 & 0.22 & 1.00 & 0.38 \\ \text { RealEst } & -0.06 & 0.06 & -0.08 & -0.09 & 0.38 & 1.00\end{array}$;




\section{Variables}

$x(i) \quad$ fraction of portfolio invested in asset $i$

ExReturn expected return on portfolio

\section{Positive Variable x;}

\section{Equations}

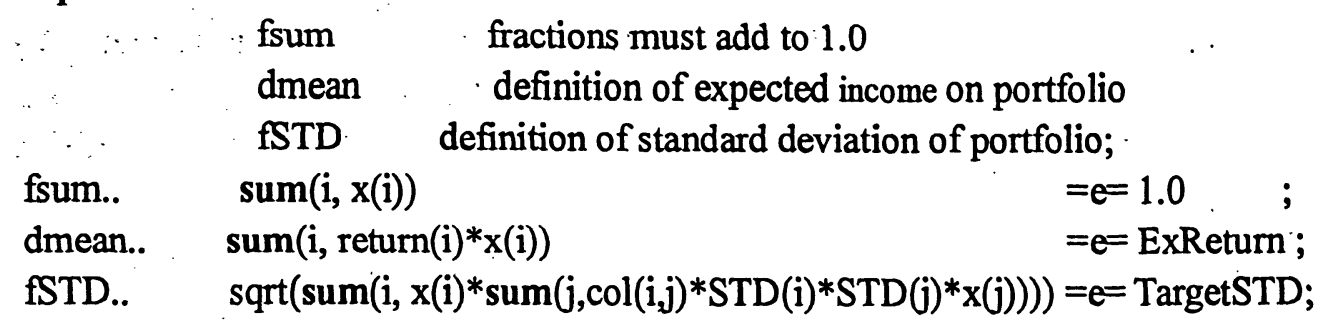

Model portfolio /all/;

Solve portfolio using nlp maximizing ExReturn; 1 .

Display ExReturn.l, targetSTD, x.l ;

* Now change the value of targetSTD

targetSTD $=$ targetSTD + 0.2;

Scalars R1;

Scalars R2;

R1 = ExReturn.1;

Model p1 /fsum, dmean, fSTD/;

Solve p1 using nlp maximizing ExReturn;

$\mathrm{R} 2=$ ExReturn.1;
$\mathrm{k}=1$

For $(k=1$ to 20 ,

If $(R 1<R 2$,

targetSTD $=$ targetSTD + 0.2 ;

$\mathrm{R} 1=\mathrm{R} 2$;

Solve p1 using nlp maximizing ExReturn;

R2 = ExReturn.1;

Else

targetSTD $=$ targetSTD -0.2 ;

Solve p1 using nlp maximizing ExReturn;

Display ExReturn.l, targetSTD, x.l ;

targetSTD $=$ targetSTD +0.2 ; 


\section{Appendix 3.4}

\section{GAMS Program for Obtaining Local Optimal Portfolio in terms of the Current Income Objective}

\section{SOnText}

This GAMS program is written to solve the quadratic programming model (Model 4.2) whose objective function is to maximize an investor's utility in terms of the current income (CRI) objective. The outputs are the characteristics of local optimal portfolio, including the expected return, standard deviation, percentage of portfolio invested in each asset class, as well as the values of the investor's risk tolerance and utility.

\section{\$OffText}

Set $i$ assets /LaStock, SmStock,LCBond, LGBond,TBill, RealEst/; alias (i,j);

Set $m$ reference portfolio A $/ \operatorname{ReA}, \operatorname{Var} A /$;

Set 1 reference portfolio $\mathrm{C} / \mathrm{ReC}, \operatorname{VarC} /$;

Scalar $t$ the investor's risk tolerance;

Scalar $k$ the relative degree of preference between portfolios $A$ and $C \quad / 0.754 /$;

* For aggressive action, $\mathrm{k}=0.754$

* For normal action, $\mathrm{k}=0.763$

* For conservative action, $\mathrm{k}=0.793$

Parameters refA(m) expected return and variance of reference portfolio $\mathrm{A}$

$$
\begin{array}{cc}
/ \operatorname{ReA} & 4.50 \\
\operatorname{VarA} & 2.65 \quad / ;
\end{array}
$$

Parameters refC(l) expected return and variance of reference portfolio $\mathrm{C}$

$$
\begin{array}{ccc}
\text { / } & \operatorname{ReC} & 5.90 \\
& \operatorname{VarC} & 12.25 / \text {; }
\end{array}
$$

$\mathrm{t}=\left(\operatorname{ref} A\left({ }^{\prime} \operatorname{Var} A^{\prime}\right)-\mathrm{k} * \operatorname{refC}\left({ }^{\prime} \operatorname{Var} C^{\prime}\right)\right) /\left(\operatorname{ref} A\left({ }^{\prime} \operatorname{ReA} A^{\prime}\right)-\mathrm{k} * \operatorname{refC}\left({ }^{\prime} \operatorname{ReC} '\right)\right)$

Parameters return(i) expected current incomes of individual assets (\%)

$\begin{array}{ll}\text { / LaStock } & 4.8 \\ \text { SmStock } & 5.0 \\ \text { LCBond } & 5.9 \\ \text { LGBond } & 4.9 \\ \text { TBill } & 3.7 \\ \text { RealEst } & 5.2\end{array}$


Parameters STD(i) standard deviations of CRI of individual assets (\%)

$\begin{array}{lll}\text { I. LaStock } & 3.3 \\ \text { SmStock } & 8.0 \\ \text { LCBond } & 3.5 \\ \text { LGBond } & 2.9 \\ \text { TBill } & 2.4 \\ \text { RealEst } & : & 3.3 \quad / \text {; }\end{array}$

Table col(i,j) correlations between assets

$\begin{array}{lcccccc} & \text { LaStock } & \text { SmStock } & \text { LCBond } & \text { LGBond } & \text { TBill } & \text { RealEst } \\ \text { LaStock } & 1.00 & 0.82 & 0.19 & 0.11 & -0.07 & -0.06 \\ \text { SmStock } & 0.82 & 1.00 & 0.08 & -0.01 & -0.08 & 0.06 \\ \text { LCBond } & 0.19 & 0.08 & 1.00 & 0.93 & 0.19 & -0.08 \\ \text { LGBond } & 0.11 & -0.01 & 0.93 & 1.00 & 0.22 & -0.09 \\ \text { TBill } & -0.07 & -0.08 & 0.19 & 0.22 & 1.00 & 0.38 \\ \text { RealEst } & -0.06 & 0.06 & -0.08 & -0.09 & 0.38 & 1.00\end{array}$
Variables $\quad x(i) \quad$ fraction of portfolio invested in asset $i$
returnEx expected return of portfolio
variance definition of portfolio variance
standev standard deviation of portfolio
utility definition of utility ;

\section{Positive Variable $\mathrm{x}$;}

\section{Equations}

fsum fractions must add to 1.0

freturnEx definition of portfolio return

fvariance definition of portfolio variance

dutil definition of utility;
fsum.. $\quad \operatorname{sum}(i, x(i))$
$=\mathrm{e}=1.0$;
freturnEx.. $\operatorname{sum}(i, \operatorname{return}(i) * x(i))$
$=\mathrm{e}=$ returnEx ;
fvariance.. $\operatorname{sum}(i, x(i) * \operatorname{sum}(j, \operatorname{col}(i, j) * \operatorname{STD}(i) * \operatorname{STD}(j) * x(j))) \quad=e=$ variance;
dutil.. returnEx - variance/t
$=\mathrm{e}=$ utility ;

Model portfolio / all /;

Solve portfolio using nlp maximizing utility;

standev.l = sqrt(variance.l) ;

Display t, utility.l, returnEx.l, standev.l, x.1; 


\section{Appendix 3.5}

\section{GAMS Program for Finding Performance of the Complete Portfolio in terms of the Current income Objective}

\section{SOnText}

This GAMS program is written to obtain the performance information (expected return and standard deviation) of the complete portfolio in terms of the current income (CRI) objective.

\section{\$OffText}

Set i assets /LaStock, SmStock,LCBond, LGBond,TBill, RealEst/; alias (i,j)

Scalar ReturnC Excepted return of complete portfolio for CRI objective VarC Variance of complete portfolio for CRI objective StdC Standard deviation of complete portfolio for CRI objective;

Parameters $x(i)$ fraction of assets making up the complete portfolio

$\begin{array}{lll}\text { LaStock } & 0.282 \\ \text { SmStock } & 0.164 \\ \text { LCBond } & 0.177 \\ \text { LGBond } & 0.000 \\ \text { TBill } & 0.040 \\ \text { RealEst } & 0.337 \quad / \text {; }\end{array}$

*These data are for normal action;

* For aggressive action, LaStock $=0.286$, SmStock $=0.266$, LCBond $=0.148$,

* $\quad$ LGBond $=0.000$, TBill $=0.009$, RealEst $=0.291$.

$*$ For conservative action, LaStock $=0.232$, SmStock $=0.081, \mathrm{LCB}$ ond $=0.283$,

* $\quad$ LGBond $=0.001, \mathrm{TBill}=0.075$, RealEst $=0.328$.

Parameters return(i) expected current incomes of individual assets (\%)

$\begin{array}{ll}\text { / LaStock } & 4.8 \\ \text { SmStock } & 5.0 \\ \text { LCBond } & 5.9 \\ \text { LGBond } & 4.9 \\ \text { TBill } & 3.7 \\ \text { RealEst } & 5.2 \quad / \text {; }\end{array}$


Parameters STD(i) standard deviations of CRI of individual assets (\%)

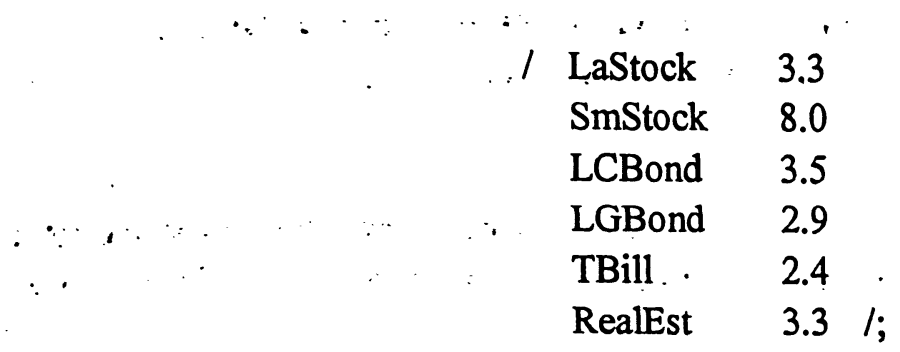

Table col(i,j) correlations between assets

$\begin{array}{lccccccc} & \text { LaStock } & \text { SmStock } & \text { LCBond } & \text { LGBond } & \text { TBill } & \text { RealEst } \\ \text { LaStock } & 1.00 & 0.82 & 0.19 & 0.11 & -0.07 & -0.06 \\ \text { SmStock } & 0.82 & 1.00 & 0.081 & -0.01 & -0.08 & 0.06 & \\ \text { LCBond } & 0.19 & 0.08 & 1.00 & 0.93 & 0.19 & -0.08 & \\ \text { LGBond } & 0.11 . & -0.01 . & 0.93 & 1.00 & 0.22 & -0.09 & \\ \text { TBill } & -0.07 & -0.08 & 0.19 & 0.22 & 1.00 & 0.38 & \\ \text { RealEst } & -0.06 & 0.06 & -0.08 & -0.09 & 0.38 & 1.00 & \text {; }\end{array}$

\footnotetext{
returnC $=\operatorname{sum}(i, \operatorname{return}(i) * x(i))$;

$\operatorname{varC}=\operatorname{sum}(i, x(i) * \operatorname{sum}(j, \operatorname{col}(i, j) * \operatorname{STD}(i) * \operatorname{STD}(j) * x(j)))$;

$\operatorname{stdC}=\operatorname{sqrt}(\operatorname{var} C)$;
}

Display ReturnC, StdC; 


\section{GAMS Program for Obtaining the Optimal Portfolio for a Given Target Return in terms of the Current Income Objective}

\section{SOnText}

This GAMS program is written to solve the quadratic programming model (Model 4.1) whose objective function is to minimize a portfolio's variance in terms of the current income (CRI) objective. The input data are the specified expected target returns. The outputs are the characteristics of optimal portfolio, including the expected return, standard deviation, percentage of portfolio invested in each asset class.

\section{\$OffText}

Set $i$ assets /LaStock, SmStock,LCBond, LGBond,TBill, RealEst/; alias (i,j) Scalar target target mean current income (CRI) of portfolio (\%) / 5.9/;

Parameters return(i) expected current incomes of individual assets (\%)

$\begin{array}{lll}\text { / LaStock } & 4.8 \\ \text { SmStock } & 5.0 \\ \text { LCBond } & 5.9 \\ \text { LGBond } & 4.9 \\ \text { TBill } & 3.7 \\ \text { RealEst } & 5.2 \quad \text { /; }\end{array}$

Parameters STD(i) standard deviations of CRI of individual assets (\%)

$\begin{array}{ll}\text { / LaStock } & 3.3 \\ \text { SmStock } & 8.0 \\ \text { LCBond } & 3.5 \\ \text { LGBond } & 2.9 \\ \text { TBill } & 2.4 \\ \text { RealEst } & 3.3 \quad / \text {; }\end{array}$

Table col $(\mathrm{i}, \mathrm{j})$ correlations between assets

$\begin{array}{lcccccc} & \text { LaStock } & \text { SmStock } & \text { LCBond } & \text { LGBond } & \text { TBill } & \text { RealEst } \\ \text { LaStock } & 1.00 & 0.82 & 0.19 & 0.11 & -0.07 & -0.06 \\ \text { SmStock } & 0.82 & 1.00 & 0.08 & -0.01 & -0.08 & 0.06 \\ \text { LCBond } & 0.19 & 0.08 & 1.00 & 0.93 & 0.19 & -0.08 \\ \text { LGBond } & 0.11 & -0.01 & 0.93 & 1.00 & 0.22 & -0.09 \\ \text { TBill } & -0.07 & -0.08 & 0.19 & 0.22 & 1.00 & 0.38 \\ \text { RealEst } & -0.06 & 0.06 & -0.08 & -0.09 & 0.38 & 1.00\end{array}$


$\begin{array}{cll}\text { Variables } & \begin{array}{l}\mathbf{x}(\mathrm{i}) \\ \text { variance }\end{array} & \begin{array}{l}\text { fraction of portfolio invested in asset } \mathrm{i} \\ \text { variance of portfolio }\end{array} \\ \because & \text { stander } & \text { standard deviation of portfolio }\end{array}$

Positive Variable $\dot{x}$;

Equations . fum ... fractions must add to 1.0

- dean definition of expected income on portfolio fvariance definition of portfolio variance;

fum.. $\quad \operatorname{sum}(i, x(i))$

dean.. $\quad \operatorname{sum}(i, \operatorname{return}(i) * x(i))$

fvariance.. $\quad \operatorname{sum}(i, x(i) * \operatorname{sum}(j, \operatorname{col}(i, j) * \operatorname{STD}(i) * \operatorname{STD}(j) * x(j)))$

Model portfolio / all / ;

Solve portfolio using nIp minimizing variance;

standev.1 = sqrt(variance.1)

Display target, variance.1, standev.l, x.l; $=\mathrm{e}=1.0$;

$=\mathrm{e}=$ target;

$\mathrm{e}=$ variance;

115 
Appendix 4

GAMS Programs Associated with the After-tax Return Objective 


\section{Appendix 4.1}

\section{GAMS Program for Finding the Global Minimum Variance Portfolio in terms of the After-tax Return Objective}

\section{SOnText}

This GAMS program is written to find the global minimum variance portfolio along efficient frontier in terms of the after-tax return (ATR) objective. The underlying quadratic programming model is Model 4.1.

\$OffText

Set $\quad i$ assets /LaStock, SmStock,LCBond, LGBond,TBill, RealEst/; alias (i,j);

Scalars $k$ index for loop target initial target return $/ 2.5 /$;

Parameters return(i) expected after-tax returns of individual assets (\%)

$\begin{array}{lr}\text { / LaStock } & 8.9 \\ \text { SmStock } & 13.3 \\ \text { LCBond } & 3.2 \\ \text { LGBond } & 2.6 \\ \text { TBill } & 2.0 \\ \text { RealEst } & 4.6 \quad / \text {; }\end{array}$

Parameters STD(i) standard deviations of ATR of individual assets (\%)

$\begin{array}{lr}\text { / LaStock } & 15.4 \\ \text { SmStock } & 26.5 \\ \text { LCBond } & 5.0 \\ \text { LGBond } & 4.2 \\ \text { TBill } & 1.8 \\ \text { RealEst } & 8.0 . / \text {; }\end{array}$

Table col( $\mathrm{i}, \mathrm{j}) \quad$ correlations between assets

$\begin{array}{lcccccc} & \text { LaStock } & \text { SmStock } & \text { LCBond } & \text { LGBond } & \text { TBill } & \text { RealEst } \\ \text { LaStock } & 1.00 & 0.82 & 0.19 & 0.11 & -0.07 & -0.06 \\ \text { SmStock } & 0.82 & 1.00 & 0.08 & -0.01 & -0.08 & 0.06 \\ \text { LCBond } & 0.19 & 0.08 & 1.00 & 0.93 & 0.19 & -0.08 \\ \text { LGBond } & 0.11 & -0.01 & 0.93 & 1.00 & 0.22 & -0.09 \\ \text { TBill } & -0.07 & -0.08 & 0.19 & 0.22 & 1.00 & 0.38 \\ \text { RealEst } & -0.06 & 0.06 & -0.08 & -0.09 & 0.38 & 1.00\end{array}$




\begin{tabular}{|c|c|c|}
\hline triables & $\mathrm{x}(\mathrm{i})$ & $\begin{array}{l}\text { fraction of portfolio invested in asset } \\
\text { variance of portfolio }\end{array}$ \\
\hline
\end{tabular}

Positive Variable x;

Equations

$\begin{array}{ll}\text { fsum } & \text { fractions must add to } 1.0 \\ \text { dmean } & \text { definition of expected return on portfolio } \\ \text { fvariance } & \text { definition of portfolio variance; }\end{array}$

fsum.. $\operatorname{sum}(\mathrm{i}, \mathrm{x}(\mathrm{i})) \quad=\mathrm{e}=1.0$;

dmean.. $\quad \operatorname{sum}(\mathrm{i}, \operatorname{return}(\mathrm{i}) * \mathrm{x}(\mathrm{i})) \quad=\mathrm{e}=$ target;

fvariance.. $\quad \operatorname{sum}(i, x(i) * \operatorname{sum}(j, \operatorname{col}(i, j) * \operatorname{STD}(i) * \operatorname{STD}(j) * x(j))) \quad=e=$ variance;

Model portfolio / all/;

Solve portfolio using nlp minimizing variance;

target $=$ target -0.1 ;

Scalars v1;

Scalars v2;

$\mathrm{v} 1=$ variance. 1

* set vl current variance

Model p1 /fsum, dmean, fvariance/;

Solve pl using nlp minimizing variance;

v2 = variance.1;

$\mathrm{k}=1$;

For $(k=1$ to 6 ,

If $(\mathrm{v} 1>\mathrm{v} 2$,

target $=$ target -0.1 ;

$\mathrm{v} 1=\mathrm{v} 2$;

Solve $\mathrm{p} 1$ using nlp minimizing variance;

v2 = variance. 1 ;

Else

target $=$ target +0.1 ;

Solve 1 using nlp minimizing variance;

standev. $1=$ sqrt(v1)

Display vl, standev.l, target ;

target $=$ target -0.1 ;

);

); 


\section{Appendix 4.2}

\section{GAMS Program for.Generating the List of Candidate Portfolios in terms of the After-tax Return Objective}

\section{\$OnText}

This GAMS program is written to generate the candidate conservative reference portfolio list in terms of the after-tax return (ATR) objective. Slightly modified, this program can also be used to generate the aggressive portfolio list.

\section{\$OffText}

Set $\quad \mathrm{i}$ assets /LaStock, SmStock,LCBond, LGBond,TBill, RealEst/; alias (i,j)

Scalar target targeted mean ATR on portfolio (\%) 12.5/;

* If generate aggressive portfolio, the target value can be set as 12 , for example.

Scalar $\mathbf{k}$ index for loop;

Parameters return(i) expected after-tax returns of individual assets (\%)

$\begin{array}{llr}\text { / LaStock } & 8.9 \\ \text { SmStock } & 13.3 \\ \text { LCBond } & 3.2 \\ \text { LGBond } & 2.6 \\ \text { TBill } & 2.0 \\ \text { RealEst } & 4.6\end{array}$

Parameters STD(i) standard deviations of ATR of individual assets (\%)

$\begin{array}{llr}\text { LaStock } & 15.4 \\ \text { SmStock } & 26.5 \\ \text { LCBond } & 5.0 \\ \text { LGBond } & 4.2 \\ \text { TBill } & 1.8 \\ \text { RealEst } & 8.0 \quad \text { /; }\end{array}$

Table col(i,j) correlations between assets

$\begin{array}{lcccccc} & \text { LaStock } & \text { SmStock } & \text { LCBond } & \text { LGBond } & \text { TBill } & \text { RealEst } \\ \text { LaStock } & 1.00 & 0.82 & 0.19 & 0.11 & -0.07 & -0.06^{\circ} \\ \text { SmStock } & 0.82 & 1.00 & 0.08 & -0.01 & -0.08 & 0.06 \\ \text { LCBond } & 0.19 & 0.08 & 1.00 & 0.93 & 0.19 & -0.08 \\ \text { LGBond } & 0.11 & -0.01 & 0.93 & 1.00 & 0.22 & -0.09 \\ \text { TBill } & -0.07 & -0.08 & 0.19 & 0.22 & 1.00 & 0.38 \\ \text { RealEst } & -0.06 & 0.06 & -0.08 & -0.09 & 0.38 & 1.00\end{array}$;




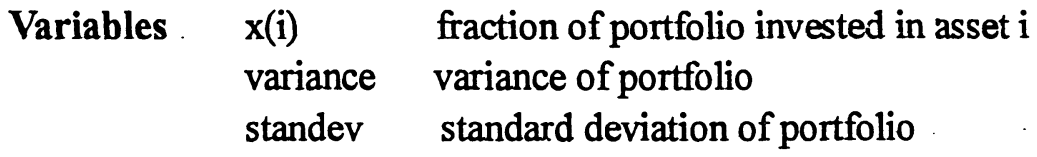

Positive Variable $x$;

Equations fsum fractions must add to 1.0

dmean definition of expected ATR on portfolio

fvariance definition of portfolio variance;

fsum..

$$
\operatorname{sum}(i, x(i))
$$$$
=\mathrm{e}=1.0 \text {; }
$$

dmean.

$\operatorname{sum}(i, \operatorname{return}(i) * x(i))$

$=\mathrm{e}=$ target ;

fvariance..

$\operatorname{sum}(i, x(i) * \operatorname{sum}(j, \operatorname{col}(i, j) * \operatorname{STD}(i) * \operatorname{STD}(j) * x(j)))=e=$ variance;

Model portfolio / all /;

Solve portfolio using nlp minimizing variance;

standev.l = sqrt(variance.l) ;

Display target, variance.l, standev.l, x.l;

For $(k=1$ to 5 ,

target $=$ target +0.5 ;

* If used for generating aggressive portfolio list, target $=$ target - step

* In this example, the length of step is set at 0.5

Solve portfolio using nlp minimizing variance; standev.1 = sqrt(variance.l) ;

Display target, variance.l, standev.l, x.l; ); 


\section{GAMS Program for Finding the Maximum Return Portfolio in terms of the After-tax Return Objective}

\$OnText

This GAMS program is written to find the maximum return portfolio along the efficient frontier in terms of the after-tax return (ATR) objective. This portfolio is considered the most aggressive. The underlying quadratic programming model is Model 2.1.

\$OffText

Set $\quad \mathrm{i}$ assets /LaStock, SmStock,LCBond, LGBond,TBill, RealEst/; alias (i,j);

Scalar $\mathrm{k}$ index for loop;

Scalar TargetSTD target STD on portfolio (\%) /24.9/;

Parameters return(i) expected after-tax returns of individual assets (\%)

\begin{tabular}{|lr} 
/ LaStock & 8.9 \\
SmStock & 13.3 \\
LCBond & 3.2 \\
LGBond & 2.6 \\
TBill & 2.0 \\
RealEst & $4.6 \quad$ /;
\end{tabular}

Parameters STD(i) standard deviations of ATR of individual assets (\%)

\begin{tabular}{|lr} 
/ LaStock & 15.4 \\
SmStock & 26.5 \\
LCBond & 5.0 \\
LGBond & 4.2 \\
TBill & 1.8 \\
RealEst & $8.0 \quad$ /;
\end{tabular}

Table col(i,j) correlations bewteen assets

\begin{tabular}{lcccccc} 
& LaStock & SmStock & LCBond & LGBond & TBill & RealEst \\
LaStock & 1.00 & 0.82 & 0.19 & 0.11 & -0.07 & -0.06 \\
SmStock & 0.82 & 1.00 & 0.08 & -0.01 & -0.08 & 0.06 \\
LCBond & 0.19 & 0.08 & 1.00 & 0.93 & 0.19 & -0.08 \\
LGBond & 0.11 & -0.01 & 0.93 & 1.00 & 0.22 & -0.09 \\
TBill & -0.07 & -0.08 & 0.19 & 0.22 & 1.00 & 0.38 \\
RealEst & -0.06 & 0.06 & -0.08 & -0.09 & 0.38 & 1.00 \\
& \multicolumn{2}{c}{ - 121} & & &
\end{tabular}


Variables . $x(i) \quad$ fraction of portfolio invested in asset $i$

ExReturn expected return on portfolio

Positive Variable x;

Equations

fsum fractions must add to 1.0

dmean definition of expected ATR on portfolio

fSTD definition of standard deviation of portfolio;

fsum.. $\quad \cdot \operatorname{sum}(i, x(i))$

$=\mathrm{e}=1.0$

dmean.. $\quad \operatorname{sum}(i, \operatorname{return}(i) * x(i)) \quad=e=$ ExReturn;

fSTD.. $\quad \operatorname{sqrt}(\operatorname{sum}(i, x(i) * \operatorname{sum}(j, \operatorname{col}(i, j) * \operatorname{STD}(i) * \operatorname{STD}(j) * x(j))))=e=$ TargetSTD;

Model portfolio / all/;

Solve portfolio using nlp maximizing ExReturn;

Display ExReturn.l, targetSTD, x.1 ;

* Now change the value of targetSTD

$\operatorname{targetSTD}=\operatorname{targetSTD}+0.2 ;$

Scalars R1;

Scalars R2 ;

R1 = ExReturn.1;

Model p1 /fsum, dmean, fSTD/;

Solve p1 using nlp maximizing ExReturn;

R2 = ExReturn.1;

$\mathrm{k}=1$;

For $(k=1$ to 20 ,

If $(\mathrm{R} 1<\mathrm{R} 2$,

targetSTD $=$ targetSTD +0.2 ;

$\mathrm{R} 1=\mathrm{R} 2$;

Solve p1 using nlp maximizing ExReturn;

R2 = ExReturn.l;

Else

targetSTD = targetSTD -0.2 ;

Solve p1 using nlp maximizing ExReturn;

Display ExReturn.1, targetSTD, x.l ;

targetSTD $=$ targetSTD +0.2 ;

);

); 


\section{Appendix 4.4}

\section{GAMS Program for Obtaining the Local Optimal Portfolio : in terms of the After-tax Return Objective}

\section{SOnText}

This GAMS program is written to solve the quadratic programming model (Model 4.2) whose objective function is to maximize an investor's utility in terms of the after-tax return (ATR) objective. The outputs are the characteristics of local optimal portfolio, including the expected return, standard deviation, percentage of portfolio invested in each asset class, as well as the values of the investor's. risk tolerance and utility.

\section{\$OffText}

Set i assets /LaStock, SmStock,LCBond, LGBond,TBill, RealEst/; alias (i,j);

Set $m$ reference portfolio $A / \operatorname{ReA}, \operatorname{Var} A /$;

Set 1 reference portfolio $\mathrm{C} / \mathrm{ReC}, \operatorname{VarC}$;

Scalar $t$ the investor's risk tolerance;

Scalar $\mathbf{k}$ the relative degree of preference between portfolios A and C / 0.630/;

* For aggressive action, $\mathrm{k}=0.475$

* For normal action, $\mathrm{k}=0.630$

* For conservative action, $\mathrm{k}=2.759$

Parameters refA(m) expected return and variance of reference portfolio $A$

$\begin{array}{ll}/ \operatorname{ReA} & 3.00 \\ \operatorname{VarA} & 5.19\end{array}$

Parameters refC(l) expected return and variance of reference portfolio $\mathrm{C}$

$$
\begin{array}{cc}
/ \operatorname{ReC} & 10.00 \\
\operatorname{VarC} & 277.89 / ;
\end{array}
$$

$\mathrm{t}=\left(\operatorname{ref} A\left({ }^{\prime} \operatorname{Var} A^{\prime}\right)-\mathrm{k} * \operatorname{refC}\left({ }^{\prime} \operatorname{Var} C^{\prime}\right)\right) /\left(\operatorname{refA}\left({ }^{\prime} \operatorname{ReA}{ }^{\prime}\right)-\mathrm{k} * \operatorname{refC}\left({ }^{\prime} \operatorname{ReC} C^{\prime}\right)\right)$

Parameters return(i) expected after-tax returns of individual assets (\%)

\begin{tabular}{llrrr} 
L LaStock & 8.9 & & \\
SmStock & 13.3 & & \\
LCBond & 3.2 & & \\
LGBond & - & 2.6 &. & \\
TBill & 2.0 & & \\
RealEst & $4.6 \%$ & \\
\hline
\end{tabular}


Parameters STD(i) standard deviations of ATR of individual assets (\%)

$\begin{array}{llr}\text { LaStock } & 15.4 \\ \text { SmStock } & 26.5 \\ \text { LCBond } & 5.0 \\ \text { LGBond } & 4.2 \\ \text { TBill } & 1.8 \\ \text { RealEst } & 8.0\end{array}$

Table col(i,j) correlations between assets

\begin{tabular}{|c|c|c|c|c|c|c|}
\hline & LaStock & SmStock & LCBond & LGBond & TBill & RealEst \\
\hline LaStock & 1.00 . & 0.82 & 0.19 & 0.11 & -0.07 & -0.06 \\
\hline SmStock & 0.82 & 1.00 & 0.08 & -0.01 & -0.08 & 0.06 \\
\hline LCBond & 0.19 & 0.08 & 1.00 & 0.93 & $0: 19$ & -0.08 \\
\hline LGBond & 0.11 & -0.01 & 0.93 & 1.00 & 0.22 & -0.09 \\
\hline TBill & -0.07 & -0.08 & 0.19 & 0.22 & 1.00 & 0.38 \\
\hline RealEst & -0.06 & 0.06 & -0.08 & -0.09 & 0.38 & 1.00 \\
\hline
\end{tabular}

Variables $\quad x(i) \quad$ fraction of portfolio invested in asset $i$

returnEx expected return of portfolio

variance definition of portfolio variance

standev standard deviation of portfolio

utility definition of utility ;

Positive Variable $x$;

Equations

fsum fractions must add to 1.0

freturnEx definition of portfolio return

fvariance definition of portfolio variance

dutil definition of utility;

fsum.. $\quad \operatorname{sum}(\mathbf{i}, x(\mathbf{i}))$

freturnEx.. $\operatorname{sum}(i, \operatorname{return}(i) * x(i))$

fvariance.. $\operatorname{sum}(i, x(i) * \operatorname{sum}(j, \operatorname{col}(i, j) * \operatorname{STD}(i) * \operatorname{STD}(j) * x(j)))$

dutil. $\quad$ returnEx - variance/t $=\mathrm{e}=1.0 ;$

$=\mathrm{e}=$ returnEx;

$=\mathrm{e}$ variance;

$=\mathrm{e}=$ utility ;

Model portfolio / all /;

Solve portfolio using nlp maximizing utility;

standev.l = sqrt(variance.l) ;

Display t, utility.1, returnEx.l, standev.l, x.1; 


\section{Appendix 4.5}

\section{GAMS Program for Finding Performance of the Complete Portfolio in terms of the After-tax Return Objective}

\section{\$OnText}

This GAMS program is written to obtain the performance information (expected return and standard deviation) of the complete portfolio in terms of the after-tax return (ATR) objective.

\section{SOffText}

Set $\mathrm{i}$ assets /LaStock, SmStock,LCBond, LGBond,TBill, RealEst/; alias (i,j)

Scalar ReturnC Excepted return of complete portfolio for ATR objective

VarC : Variance of complete portfolio for ATR objective

StdC Standard deviation of complete portfolio for ATR objective;

Parameters $\mathrm{x}(\mathrm{i})$ fraction of assets making up the complete portfolio

$\begin{array}{lll}\text { / LaStock } & 0.282 \\ \text { SmStock } & 0.164 \\ \text { LCBond } & 0.177 \\ \text { LGBond } & 0.000 \\ \text { TBill } & 0.040 \\ . \quad \text { RealEst } & 0.337 \quad / \text {; }\end{array}$

*These data are for normal action;

$*$ For aggressive action, LaStock $=0.286$, SmStock $=0.266, \mathrm{LCBond}=0.148$,

* $\quad$ LGBond $=0.000$, TBill $=0.009$, RealEst $=0.291$.

$*$ For conservative action, LaStock $=0.232$, SmStock $=0.081, \mathrm{LCBond}=0.283$,

$$
\text { LGBond }=0.001, \text { TBill }=0.075 \text {, RealEst }=0.328 \text {. }
$$

Parameters return(i) expected after-tax returns of individual assets (\%)

$\begin{array}{llr}\text { / LaStock } & 8.9 \\ \text { SmStock } & 13.3 \\ \text { LCBond } & 3.2 \\ \text { LGBond } & 2.6 \\ \text { TBill } & 2.0 \\ \text { RealEst } & 4.6 \quad \text { /; }\end{array}$


Parameters STD(i) standard deviations of ATR of individual assets (\%)

$\begin{array}{lr}\text { LaStock } & 15.4 \\ \text { SmStock } & 26.5 \\ \text { LCBond } & 5.0 \\ \text { LGBond } & 4.2 \\ \text { TBill } & 1.8 \\ \text { RealEst } & 8.0 \quad / \text {; }\end{array}$

Table col(i,j) correlations between assets

$\begin{array}{lcccccc} & \text { LaStock } & \text { SmStock } & \text { LCBond } & \text { LGBond } & \text { TBill } & \text { RealEst } \\ \text { LaStock } & 1.00 & 0.82 & 0.19 & 0.11 & -0.07 & -0.06 \\ \text { SmStock } & 0.82 & 1.00 & 0.08 & -0.01 & -0.08 & 0.06 \\ \text { LCBond } & 0.19 & 0.08 & 1.00 & 0.93 & 0.19 & -0.08 \\ \text { LGBond } & 0.11 & -0.01 & 0.93 & 1.00 & 0.22 & -0.09 \\ \text { TBill } & -0.07 & -0.08 & 0.19 & 0.22 & 1.00 & 0.38 \\ \text { RealEst } & -0.06 & 0.06 & -0.08 & -0.09 & 0.38 & 1.00\end{array}$

returnC $=\operatorname{sum}(i, \operatorname{return}(i) * x(i))$;

$\operatorname{varC}=\operatorname{sum}(i, x(i) * \operatorname{sum}(j, \operatorname{col}(i, j) * \operatorname{STD}(i) * \operatorname{STD}(j) * x(j))) ;$

stdC $=\operatorname{sqrt}(\operatorname{var} C)$;

Display ReturnC, StdC; 


\section{Appendix 4.6}

\section{GAMS Program for Obtaining the Optimal-Portfolio for a Given Target Return in terms of the After-tax Return Objective}

\section{SOnText}

This GAMS program is written for solving the quadratic programming model (Model 4.1) which objective is to minimize a portfolio's expected variance in terms of the after-tax return (ATR) objective. The input data are the specified expected target returns. The outputs are the characteristics of optimal portfolio, including the expected return, standard deviation, percent of each asset class invested.

\section{\$OffText}

Set i assets /LaStock, SmStock,LCBond, LGBond,TBill, RealEst/; alias (i,j) Scalar target target mean ATR on portfolio (\%) / 10/;

Parameters return(i) expected after-tax returns of individual assets (\%)

$\begin{array}{llr}\text { / LaStock } & 8.9 \\ \text { SmStock } & 13.3 \\ \text { LCBond } & 3.2 \\ \text { LGBond } & 2.6 \\ \text { TBill } & 2.0 \\ \text { RealEst } & 4.6 \quad / \text {; }\end{array}$

Parameters STD(i) standard deviations of individual assets (\%)

$\begin{array}{lr}\text { / LaStock } & 15.4 \\ \text { SmStock } & 26.5 \\ \text { LCBond } & 5.0 \\ \text { LGBond } & 4.2 \\ \text { TBill } & 1.8 \\ \text { RealEst } & 8.0 \quad / \text {; }\end{array}$

Table $\operatorname{col}(\mathrm{i}, \mathrm{j})$ correlations between assets

$\begin{array}{lcccccc} & \text { LaStock } & \text { SmStock } & \text { LCBond } & \text { LGBond } & \text { TBill } & \text { RealEst } \\ \text { LaStock } & 1.00 & 0.82 & 0.19 & 0.11 & -0.07 & -0.06 \\ \text { SmStock } & 0.82 & 1.00 & 0.08 & -0.01 & -0.08 & 0.06 \\ \text { LCBond } & 0.19 & 0.08 & 1.00 & 0.93 & 0.19 & -0.08 \\ \text { LGBond } & 0.11 & -0.01 & 0.93 & 1.00 & 0.22 & -0.09 \\ \text { TBill } & -0.07 & -0.08 & 0.19 & 0.22 & 1.00 & 0.38 \\ \text { RealEst } & -0.06 & 0.06 & -0.08 & -0.09 & 0.38 & 1.00\end{array}$


Variables $\quad x(i) \quad$ fraction of portfolio invested in asset $i$

variance variance of portfolio

standev standard deviation of portfolio

\section{Positive Variable $\mathrm{x}$;}

Equations fsum fractions must add to 1.0

dmean definition of expected income on portfolio

fvariance definition of portfolio variance;

fsum.. $\quad \operatorname{sum}(i, x(i))$

dmean. $\quad \operatorname{sum}(i, \operatorname{return}(i) * x(i))$

fvariance.. $=\mathrm{e}=1.0$;

$=\mathrm{e}=$ target;

$\operatorname{sum}(i, x(i) * \operatorname{sum}(j, \operatorname{col}(i, j) * \operatorname{STD}(i) * \operatorname{STD}(j) * x(j)))$

$=\mathrm{e}=$ variance;

Model portfolio / all/;

Solve portfolio using nip minimizing variance;

standev.l = sqrt(variance.l);

Display target, variance.l, standev.l, x.l; 
GAMS Programs Associated with the Preservation of Purchasing Power Objective

Appendix 5 


\section{Appendix 5.1}

\section{GAMS Program for Finding the Global Minimum Variance Portfolio in terms of the Preservation of Purchasing Power Objective}

\section{\$OnText}

This GAMS program is written to find the global minimum variance portfolio along efficient frontier in terms of the preservation of purchasing power objective (PP). The underlying quadratic programming model is Model 4.1.

\section{\$OffText}

Set $\quad \mathrm{i}$ assets /LaStock, SmStock,LCBond,LGBond,TBill, RealEst/; alias (i,j);

Scalars $\mathrm{k}$ index for loop target /1.5/;

Parameters return(i) expected inflation-adjusted returns of individual assets (\%)

$\begin{array}{lr}\text { LaStock } & 8.8 \\ \text { SmStock } & 14.3 \\ \text { LCBond } & 2.4 \\ \text { LGBond } & 1.7 \\ \text { TBill } & 0.5 \\ \text { RealEst } & 4.3 \quad / \text {; }\end{array}$

Parameters STD(i) standard deviations of individual assets (\%)

$\begin{array}{lr}\text { LaStock } & 21.1 \\ \text { SmStock } & 34.9 \\ \text { LCBond } & 10.0 \\ \text { LGBond } & 8.5 \\ \text { TBill } & 4.4 \\ \text { RealEst } & 15.0 \quad / \text {; }\end{array}$

Table col( $(\mathrm{i}, \mathrm{j})$ correlations between assets

$\begin{array}{lcccccc} & \text { LaStock } & \text { SmStock } & \text { LCBond } & \text { LGBond } & \text { TBill } & \text { RealEst } \\ \text { LaStock } & 1.00 & 0.82 & 0.19 & 0.11 & -0.07 & -0.06 \\ \text { SmStock } & 0.82 & 1.00 & 0.08 & -0.01 & -0.08 & 0.06 \\ \text { LCBond } & 0.19 & 0.08 & 1.00 & 0.93 & 0.19 & -0.08 \\ \text { LGBond } & 0.11 & -0.01 & 0.93 & 1.00 & 0.22 & -0.09 \\ \text { TBill } & -0.07 & -0.08 & 0.19 & 0.22 & 1.00 & 0.38 \\ \text { RealEst } & -0.06 & 0.06 & -0.08 & -0.09 & 0.38 & 1.00\end{array}$


Variables $x(i) \quad$ fraction of portfolio invested in asset $i$

variance variance of portfolio

standev standard deviation of portfolio

Positive Variable.x;

Equations

fsum .. fractions must add to 1.0

dmean - definition of mean expected return on portfolio

fvariance definition of portfolio variance;

fsum.. $\quad \operatorname{sum}(i, x(i))$

dmean..

$\operatorname{sum}(i, \operatorname{return}(i) * x(i))$

$=\mathrm{e}=1.0 ;$

fvariance..

$\cdot \operatorname{sum}(i, x(i) * \operatorname{sum}(j, \operatorname{col}(i, j) * \operatorname{STD}(i) * \operatorname{STD}(j) * x(j)))$

$=\mathrm{e}=$ target;

$=\mathrm{e}=$ variance;

Model portfolio / all /;

Solve portfolio using nlp minimizing variance;

target $=$ target -0.1 ;

Scalars v1;

Scalars v2 ;

$\mathrm{v} 1=$ variance. 1 ;

* set v1 current variance

Model p1 /fsum, dmean, fvariance/;

Solve 11 using nlp minimizing variance;

v2 = variance.l;

$\mathrm{k}=1$;

For $(k=1$ to 6 ,

If $(\mathrm{v} 1>\mathrm{v} 2$,

target $=$ target -0.1 ;

$\mathrm{v} 1=\mathrm{v} 2 ;$

Solve $\mathrm{pl}$ using nlp minimizing variance;

v2 = variance.l;

Else

target $=$ target +0.1 ;

Solve 1 using nlp minimizing variance;

standev. $1=$ sqitt(v1)

Display v1, standev.l, target ;

target $=$ target -0.1 ;

); 


\section{Appendix 5.2}

\section{GAMS Program for Generating the List of Candidate Portfolios in terms of the Preservation of Purchasing Power Objective}

\section{\$OnText}

This GAMS program is written to generate the candidate conservative reference portfolio list in terms of the preservation of purchasing power objective (PP). Slightly modified, this program can also be used to generate the aggressive portfolio list.

\section{\$OffText}

Set i assets /LaStock, SmStock,LCBond,LGBond,TBill, RealEst/; alias (i,j) Scalar target targeted mean return on portfolio (\%) /1.0/;

* If generate aggressive portfolio, the target value can be set as 12 , for example. Scalar $\mathrm{k}$ index for loop;

Parameters return(i) expected inflation-adjusted returns of individual assets (\%)

$\begin{array}{lr}\text { LaStock } & 8.8 \\ \text { SmStock } & 14.3 \\ \text { LCBond } & 2.4 \\ \text { LGBond } & 1.7 \\ \text { TBill } & 0.5 \\ \text { RealEst } & 4.3 \quad / \text {; }\end{array}$

Parameters STD(i) standard deviations of individual assets (\%)

$\begin{array}{lr}\text { LaStock } & 21.1 \\ \text { SmStock } & 34.9 \\ \text { LCBond } & 10.0 \\ \text { LGBond } & 8.5 \\ \text { TBill } & 4.4 \\ \text { RealEst } & 15.0 \quad / \text {; }\end{array}$

Table col(i,j) correlations between assets

$\begin{array}{lcccccc} & \text { LaStock } & \text { SmStock } & \text { LCBond } & \text { LGBord } & \text { TBill } & \text { RealEst } \\ \text { LaStock } & 1.00 & 0.82 & 0.19 & 0.11 & -0.07 & -0.06 \\ \text { SmStock } & 0.82 & 1.00 & 0.08 & -0.01 & -0.08 & 0.06 \\ \text { LCBond } & 0.19 & 0.08 & 1.00 & 0.93 & 0.19 & -0.08 \\ \text { LGBond } & 0.11 & -0.01 & 0.93 & 1.00 & 0.22 & -0.09 \\ \text { TBill } & -0.07 & -0.08 & 0.19 & 0.22 & 1.00 & 0.38 \\ \text { RealEst } & -0.06 & 0.06 & -0.08 & -0.09 & 0.38 & 1.00\end{array}$




$\begin{array}{lll}\text { Variables } & \begin{array}{l}\mathrm{x}(\mathrm{i}) \\ \text { variance }\end{array} & \begin{array}{l}\text { fraction of portfolio invested in asset } \mathrm{i} \\ \text { variance of portfolio }\end{array} \\ & \text { standev } & \text { standard deviation of portfolio }\end{array}$

Positive Variable $\mathrm{x}$;

$\begin{array}{lll}\text { Equations fsum } & \text { fractions must add to } 1.0 \\ \ddots & \text { dmean } & \text { definition of expected return of portfolio } \\ & \text { dvariance } & \text { definition of portfolio variance; }\end{array}$

fsum.. $\quad \operatorname{sum}(i, x(i)) \quad=e=1.0$;

dmean... $\quad \operatorname{sum}(\mathrm{i}, \operatorname{return}(\mathrm{i}) * \mathrm{x}(\mathrm{i})) \quad \quad \quad \quad=\mathrm{e}=$ target $;$

fvariance.. $\quad \operatorname{sum}(i, x(i) * \operatorname{sum}(j, \operatorname{col}(i, j) * \operatorname{STD}(i) * \operatorname{STD}(j) * x(j)))=e=$ variance;

Model portfolio / all/;

Solve portfolio using nlp minimizing variance;

standev.1 = sqrt(variance.l);

Display target, variance.l, standev.1, x.l;

For $(k=1$ to 5 , target $=$ target +0.5

* If used for generating aggressive portfolio list, target $=$ target - step

* In this example, the length of step is set at 0.5

Solve portfolio using nlp minimizing variance; standev. 1 = sqrt(variance. 1$)$;

Display target, variance.l, standev.l, x.l; ); 


\section{Appendix 5.3}

GAMS Program for Finding the Maximum Return Portfolio. in terms of the Preservation of Purchasing Power Objective

\$OnText

This GAMS program is written to find the maximum return portfolio along the efficient frontier in terms of the preservation of purchasing power objective (PP). This portfolio is considered the most aggressive. The underlying quadratic programming model is Model 2.1.

\section{\$OffText}

Set i assets /LaStock, SmStock,LCBond, LGBond,TBill, RealEst/; alias (i,j);

Scalar $\mathrm{k}$ index for loop;

Scalar TargetSTD target STD on portfolio (\%) / 30.9/;

Parameters return(i) expected inflation-adjusted returns of individual assets (\%)

$\begin{array}{lr}\text { LaStock } & 8.8 \\ \text { SmStock } & 14.3 \\ \text { LCBond } & 2.4 \\ \text { LGBond } & 1.7 \\ \text { TBill } & 0.5 \\ \text { RealEst } & 4.3 \quad \text { /; }\end{array}$

Parameters STD(i) standard deviations of individual assets (\%)

$\begin{array}{lr}\text { LaStock } & 21.1 \\ \text { SmStock } & 34.9 \\ \text { LCBond } & 10.0 \\ \text { LGBond } & 8.5 \\ \text { TBill } & 4.4 \\ \text { RealEst } & 15.0 \quad \text {; }\end{array}$

Table col $(\mathrm{i}, \mathrm{j}) \quad$ correlations between assets

$\begin{array}{lcccccc} & \text { LaStock } & \text { SmStock } & \text { LCBond } & \text { LGBond } & \text { TBill } & \text { RealEst } \\ \text { LaStock } & 1.00 & 0.82 & 0.19 & 0.11 & -0.07 & -0.06 \\ \text { SmStock } & 0.82 & 1.00 & 0.08 & -0.01 & -0.08 & 0.06 \\ \text { LCBond } & 0.19 & 0.08 & 1.00 & 0.93 & 0.19 & -0.08 \\ \text { LGBond } & 0.11 & -0.01 & 0.93 & 1.00 & 0.22 & -0.09 \\ \text { TBill } & -0.07 & -0.08 & 0.19 & 0.22 & 1.00 & 0.38 \\ \text { RealEst } & -0.06 & 0.06 & -0.08 & -0.09 & 0.38 & 1.00\end{array}$;




$$
\begin{array}{lll}
\text { Variables } & x(\mathrm{i}) & \text { fraction of portfolio invested in asset } i \\
\text { ExReturn } & \text { expected return on portfolio }
\end{array}
$$

Positive Variable x;

Equations

fsum fractions must add to 1.0

dmean definition of expected return on portfolio

fSTD definition of standard deviation of portfolio;

fsum.. $\quad \operatorname{sum}(i, x(i))$

dmean.. $\quad \operatorname{sum}(i, \operatorname{return}(i) * x(i)) \quad=e=$ ExReturn ;

fSTD.. $\quad \operatorname{sqrt}(\operatorname{sum}(i, x(i) * \operatorname{sum}(j, \operatorname{col}(i, j) * S T D(i) * \operatorname{STD}(j) * x(j))))=e=$ TargetSTD;

Model portfolio / all /;

Solve portfolio using nlp maximizing ExReturn;

Display ExReturn.1, targetSTD, x.l ;

* Now change the value of targetSTD

targetSTD $=\operatorname{targetSTD}+0.2$;

Scalars R1;

Scalars R2;

R1 = ExReturn.l;

Model p1 /fsum, dmean, fSTD/;

Solve p1 using nlp maximizing ExReturn;

$\mathrm{R} 2=$ ExReturn.1;
$\mathrm{k}=1 ;$

For $(k=1$ to 20 ,

If $(R 1<R 2$,

targetSTD $=$ targetSTD + 0.2 ;

$\mathrm{R} 1=\mathrm{R} 2 \quad$;

Solve p1 using nlp maximizing ExReturn;

R2 = ExReturn.1;

Else

targetSTD $=$ targetSTD $-0.2 ;$

Solve pl using nlp maximizing ExReturn;

Display ExReturn.1, targetSTD, x.1 ;

targetSTD $=$ targetSTD +0.2 ;

) ;

); 


\section{Appendix 5.4}

\section{GAMS Program for Obtaining the Local Optimal Portfolio in terms of the Preservation of Purchasing Power Objective}

\section{\$OnText}

This GAMS program is written to solve the quadratic programming model (Model 4.2) whose objective function is to maximize an investor's utility in terms of the preservation of purchasing power objective (PP). The outputs are the characteristics of local optimal portfolio, including the expected return, standard deviation, percentage of portfolio invested in each asset class, as well as the values of the investor's risk tolerance and utility.

\section{\$OffText}

Set $\mathrm{i}$ assets /LaStock, SmStock,LCBond, LGBond,TBill, RealEst/; alias (i,j);

Set $m$ reference portfolio $A / \operatorname{ReA}, \operatorname{Var} A /$;

Set 1 reference portfolio $\mathrm{C} / \mathrm{ReC}, \operatorname{VarC}$;

Scalar $\mathrm{t}$ the investor's risk tolerance;

Scalar $\mathrm{k}$ the relative degree of preference between portfolios A and C / $0.630 i$;

* $\quad$ For aggressive action, $\mathrm{k}=0.475$

* For normal action, $\mathrm{k}=0.630$

* $\quad$ For conservative action, $\mathrm{k}=2.759$

Parameters refA(m) expected return and variance of reference portfolio A / ReA 2.00

VarA $20.83 \quad / ;$

Parameters refC(l) expected return and variance of reference portfolio $\dot{C}$

/. $\operatorname{ReC} \quad 9.00$

VarC $331.24 /$

$\mathrm{t}=\left(\operatorname{ref} A\left({ }^{\operatorname{Var}} \mathrm{A}^{\prime}\right)-\mathrm{k} * \operatorname{refC}\left({ }^{\prime} \operatorname{Var} C^{\prime}\right)\right) /\left(\operatorname{refA}\left({ }^{\prime} \operatorname{ReA}{ }^{\prime}\right)-\mathrm{k} * \operatorname{refC}\left({ }^{\prime} \operatorname{ReC} '\right)\right)$;

Parameters return(i) expected inflation-adjusted returns of individual assets (\%)

$\begin{array}{llr}\text { I LaStock } & 8.8 \\ \text { SmStock } & 14.3 \\ \text { LCBond } & 2.4 \\ \text { LGBond } & 1.7 \\ \text { TBill } & 0.5 \\ \text { RealEst } & 4.3 \quad / \text {; }\end{array}$


Parameters STD(i) standard deviations of individual assets (\%)

$\begin{array}{lr}\text { 1. LaStock - } & 21.1 \\ \text { SmStock } & 34.9 \\ \text { LCBond } & 10.0 \\ \text { LGBond } & 8.5 \\ \text { TBill } & 4.4 \\ \text { RealEst } & 15.0 \quad /\end{array}$

Table col(i,j) correlations between assets

$\begin{array}{lccccccc} & \text { LaStock } & \text { SmStock } & \text { LCBond } & \text { LGBond } & \text { TBill } & \text { RealEst } & \\ \text { LaStock } & 1.00 & 0.82 & 0.19 & 0.11 & -0.07 & -0.06 & \\ \text { SmStock } & 0.82 & 1.00 & 0.08 & -0.01 & -0.08 & 0.06 & \\ \text { LCBond } & 0.19 & 0.08 & 1.001 & 0.93 & 0.19 & -0.08 & \\ \text { LGBond } & 0.11 . & -0.01 & 0.93 & 1.00 & 0.22 & -0.09 & \\ \text { TBill } & -0.07 & -0.08 & 0.19 & 0.22 & -1.00 & 0.38 & \\ \text { RealEst } & -0.06 & 0.06 & -0.08 & -0.09 & 0.38 & 1.00 & \\ & & & & & & \end{array}$

Variables $x(i) \quad$ fraction of portfolio invested in asset $i$

returnEx expected return of portfolio

variance definition of portfolio variance

standev standard deviation of portfolio

utility definition of utility ;

Positive Variable $\mathrm{x}$;

Equations

fsum fractions must add to 1.0 :

freturnEx definition of portfolio return

fvariance definition of portfolio variance

dutil definition of utility;

fsum.. $\quad \operatorname{sum}(\mathrm{i}, \mathrm{x}(\mathrm{i})) \quad . \quad=\mathrm{e}=1.0$;

freturnEx.: $\operatorname{sum}(\mathrm{i}, \operatorname{return}(\mathrm{i}) * \mathrm{x}(\mathrm{i})) \quad \ldots \quad \therefore \quad \therefore \quad=\mathrm{e}=\mathrm{returnEx}$;

fvariance.. $\operatorname{sum}(i, x(i) * \operatorname{sum}(j, \operatorname{col}(i, j) * \operatorname{STD}(i) * \operatorname{STD}(j) * x(j))) \quad=e=$ variance;

dutil. returnEx - variance/t $\quad=\mathrm{e}=$ utility ;

Model portfolio / all/;

Solve portfolio using nlp maximizing utility;

standev. 1 = sqrt(variance.l) ;

Display t, utility.1, returnEx.1, - standev.1, x.1; 


\section{Appendix 5.5}

\section{GAMS Program for Finding Performance of the Complete Portfolio in terms of the Preservation of Purchasing Power Objective}

\section{SOnText}

This GAMS program is written to obtain the performance information (expected return and standard deviation) of the complete portfolio in terms of the preservation of purchasing power objective (PP).

\section{\$OffText}

Set i assets /LaStock, SmStock,LCBond, LGBond,TBill, RealEst/; alias (i,j)

Scalar ReturnC Excepted return of complete portfolio for PP objective

VarC Variance of complete portfolio for PP objective

StdC Standard deviation complete portfolio for PP objective;

Parameters $\mathrm{x}(\mathrm{i})$ fraction of assets making up the complete portfolio

\begin{tabular}{|ll} 
/ LaStock & 0.282 \\
SmStock & 0.164 \\
LCBond & 0.177 \\
LGBond & 0.000 \\
TBill & 0.040 \\
RealEst & $0.337 \quad$ /;
\end{tabular}

*These data are for normal action;

* For aggressive action, LaStock $=0.286$, SmStock $=0.266, \mathrm{LCBond}=0.148$,

* LGBond $=0.000$, TBill $=0.009$, RealEst $=0.291$.

* For conservative action, LaStock $=0.232$, SmStock $=0.081, \mathrm{LCBond}=0.283$,

$* \quad$ LGBond $=0.001$, TBill $=0.075$, RealEst $=0.328$.

Parameters return(i) expected return on individual asset (\%)

$\begin{array}{lr}\text { / LaStock } & 8.8 \\ \text { SmStock } & 14.3 \\ \text { LCBond } & 2.4 \\ \text { LGBond } & 1.7 \\ \text { TBill } & 0.5 \\ \text { RealEst } & 4.3 \text { /; }\end{array}$


Parameters STD(i) standard deviations of individual assets (\%)

$\begin{array}{lrr}\text { / LaStock } & 21.1 \\ \text { SmStock } & 34.9 \\ \text { LCBond } & 10.0 \\ \text { LGBond } & 8.5 \\ \text { TBill } & 4.4 \\ \text { RealEst } & 15.0 \quad / \text {; }\end{array}$

Table col(i,j) correlations between assets

\begin{tabular}{lccccccc} 
& LaStock & SmStock & LCBond & LGBond & \multicolumn{2}{c}{ TBill. } & RealEst \\
LaStock & 1.00 & 0.82 & 0.19 & 0.11 & -0.07 & -0.06 \\
SmStock & 0.82 & 1.00 & 0.08 & -0.01 & -0.08 & 0.06 \\
LCBond & 0.19 & 0.08 & $1.00^{-1}$ & 0.93 & 0.19 & -0.08 \\
LGBond & 0.11 & -0.01 & 0.93 & 1.00 & 0.22 & -0.09 \\
TBill & -0.07 & -0.08 & 0.19 & 0.22 & $:$ & 1.00 & 0.38 \\
RealEst & -0.06 & 0.06 & -0.08 & -0.09 & 0.38 & 1.00
\end{tabular}

returnC $=\operatorname{sum}(i, \operatorname{return}(i) * x(i))$

$\operatorname{varC}=\operatorname{sum}(i, x(i) * \operatorname{sum}(j, \operatorname{col}(i, j) * \operatorname{STD}(i) * \operatorname{STD}(j) * x(j)))$;

stdC $=\operatorname{sqrt}(\operatorname{varC})$;

Display ReturnC, StdC; 


\section{Appendix 5.6}

GAMS Program for Obtaining the Optimal Portfolio for a Given Target Return in terms of the Preservation of Purchasing Power Objective

\section{SOnText}

This GAMS program is written to solve the quadratic programming model (Model 4.1) whose objective function is to minimize a portfolio's variance in terms of the preservation of purchasing power objective (PP). The input data are the specified expected target returns. The outputs are the characteristics of optimal portfolio, including the expected return, standard deviation, percentage of portfolio invested in each asset class.

SOffText

Set i assets /LaStock, SmStock,LCBond, LGBond,TBill, RealEst/; alias (i,j) Scalar target target mean inflation-adjusted return of portfolio (\%)/10/;

Parameters return(i) expected inflation-adjusted return of individual assets (\%)

$\begin{array}{lr}\text { LaStock } & 8.8 \\ \text { SmStock } & 14.3 \\ \text { LCBond } & 2.4 \\ \text { LGBond } & 1.7 \\ \text { TBill } & 0.5 \\ \text { RealEst } & 4.3 \quad / \text {; }\end{array}$

Parameters STD(i) standard deviations of individual assets (\%)

$\begin{array}{lr}\text { / LaStock } & 21.1 \\ \text { SmStock } & 34.9 \\ \text { LCBond } & 10.0 \\ \text { LGBond } & 8.5 \\ \text { TBill } & 4.4 \\ \text { RealEst } & 15.0\end{array}$

Table col(i,j) correlations between assets

$\begin{array}{lcccccc} & \text { LaStock } & \text { SmStock } & \text { LCBond } & \text { LGBond } & \text { TBill } & \text { RealEst } \\ \text { LaStock } & 1.00 & 0.82 & 0.19 & 0.11 & -0.07 & -0.06 \\ \text { SmStock } & 0.82 & 1.00 & 0.08 & -0.01 & -0.08 & 0.06 \\ \text { LCBond } & 0.19 & 0.08 & 1.00 & 0.93 & 0.19 & -0.08 \\ \text { LGBond } & 0.11 & -0.01 & 0.93 & 1.00 & 0.22 & -0.09 \\ \text { TBill } & -0.07 & -0.08 & 0.19 & 0.22 & 1.00 & 0.38 \\ \text { RealEst } & -0.06 & 0.06 & -0.08 & -0.09 & 0.38 & 1.00 \\ & & & 140 & & . & \end{array}$


Variables $\quad x(i) \quad$ fraction of portfolio invested in asset $i$

variance variance of portfolio

standev standard deviation of portfolio

Positive Variable x;

Equations fsum fractions must add to 1.0

dmean definition of expected return on portfolio.

fvariance definition of portfolio variance;

fsum.. $\quad \operatorname{sum}(i, x(i))$

dmean.. $\quad \operatorname{sum}(\mathbf{i}$, return(i)*x(i))

$=\mathrm{e}=1.0 ;$

fvariance.. $\quad \operatorname{sum}(i, x(i) * \operatorname{sum}(j, \operatorname{col}(i, j) * \operatorname{STD}(i) * \operatorname{STD}(j) * x(j)))$

$=\mathrm{e}=$ target;

$=\mathrm{e}=$ variance;

Model portfolio / all/; .

Solve portfolio using nlp minimizing variance;

standev.1 = sqrt(variarte. 1$)$;

Display target, variance.1, standev.l, x.l; 
Appendix 6

GAMS Programs Associated with the Liquidity Objective 


\section{Appendix 6.1}

\section{GAMS Program for Finding the Global Minimum Variance Portfolio in terms of the Liquidity Objective}

\section{\$OnText}

This GAMS program is written to find the global minimum variance portfolio along efficient frontier in terms of the liquidity objective (LD). The underlying quadratic programming model is Model 4.1.

\section{\$OffText}

Set i assets /LaStock, SmStock,LCBond, LGBond,TBill, RealEst/; alias (i,j);

Scalars $\mathrm{k}$ index for loop target $/ 0.5 /$;

Parameters return(i) expected liquidity-adjusted returns of individual assets (\%)

$\begin{array}{llr}\text { / LaStock } & 11.9 \\ \text { SmStock } & 13.4 \\ \text { LCBond } & 4.8 \\ \text { LGBond } & 4.2 \\ \text { TBill } & 3.6 \\ \text { RealEst } & 0.0 \quad / \text {; }\end{array}$

Parameters STD(i) standard deviations of individual assets (\%)

$\begin{array}{lr}\text { LaStock } & 20.5 \\ \text { SmStock } & 26.7 \\ \text { LCBond } & 7.5 \\ \text { LGBond } & 6.7 \\ \text { TBill } & 3.3 \\ \text { RealEst } & 0.0 \quad / \text {; }\end{array}$

Table col( $(\mathrm{i}, \mathrm{j})$ correlations between assets

$\begin{array}{lcccccc} & \text { LaStock } & \text { SmStock } & \text { LCBond } & \text { LGBond } & \text { TBill } & \text { RealEst } \\ \text { LaStock } & 1.00 & 0.82 & 0.19 & 0.11 & -0.07 & -0.06 \\ \text { SmStock } & 0.82 & 1.00 & 0.08 & -0.01 & -0.08 & 0.06 \\ \text { LCBond } & 0.19 & 0.08 & 1.00 & 0.93 & 0.19 & -0.08 \\ \text { LGBond } & 0.11 & -0.01 & 0.93 & 1.00 & 0.22 & -0.09 \\ \text { TBill } & -0.07 & -0.08 & 0.19 & 0.22 & 1.00 & 0.38 \\ \text { RealEst } & -0.06 & 0.06 & -0.08 & -0.09 & 0.38 & 1.00\end{array}$;


Variables $\quad x(i) \quad$ fraction of portfolio invested in asset $i$

variance variance of portfolio

standev standard deviation of portfolio

Positive Variable x;

Equations

\begin{tabular}{|c|c|c|c|c|}
\hline & $\begin{array}{l}\text { fsum } \\
\text { dmean } \\
\text { fvariance }\end{array}$ & $\begin{array}{l}\text { fractions must add to } 1.0 \\
\text { definition of expected return on } p \\
\text { definition of portfolio variance; }\end{array}$ & olio & \\
\hline . & $\begin{array}{c}\operatorname{sum}(i, x(i)) \\
\operatorname{sum}(i, \operatorname{return}(i) * \\
\operatorname{sum}(i, x(i) * \text { sum }\end{array}$ & $\begin{array}{l}\text { (i)) } \\
\operatorname{col}(\mathrm{i}, \mathrm{j}) * \operatorname{STD}(\mathrm{i}) * \operatorname{STD}(\mathrm{j}) * x(\mathrm{j})))\end{array}$ & $\begin{array}{l}=\mathrm{e}= \\
=\mathrm{e}= \\
=\mathrm{e}=\end{array}$ & $\begin{array}{l}1.0 ; \\
\text { target; } \\
\text { varianc }\end{array}$ \\
\hline
\end{tabular}

Model portfolio / all /;

Solve portfolio using nlp minimizing variance;

target $=$ target -0.1 ;

Scalars vl;

Scalars v2;

$\mathrm{v1}=$ variance.l;

* set v1 current variance

Model p1 /fsum, dmean, fvariance/;

Solve $\mathrm{p} 1$ using nlp minimizing variance;

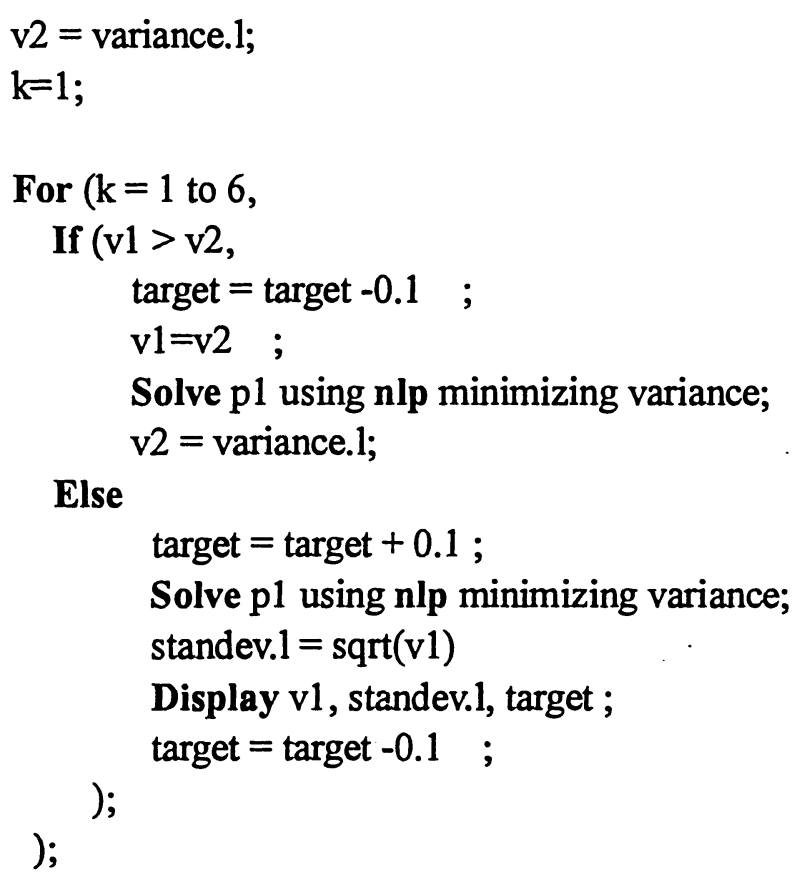




\section{Appendix 6.2}

\section{GAMS Program for Generating the List of Candidate Portfolios in terms of the Liquidity Objective}

\section{\$OnText}

This GAMS program is written to generate the candidate conservative reference portfolio list in terms of the liquidity objective (LD). Slightly modified, this program can also be used to generate the aggressive portfolio list.

\section{\$OffText}

Set $\quad \mathrm{i}$ assets /LaStock, SmStock,LCBond, LGBond,TBill, RealEst/; alias (i,j) Scalar target targeted mean return on portfolio (\%) $12.0 /$;

* If generate aggressive portfolio, the target value can be set as 12 , for example. Scalar $\mathrm{k}$ index for loop;

Parameters return(i) expected liquidity-adjusted returns of individual assets (\%)

$\begin{array}{lr}\text { LaStock } & 11.9 \\ \text { SmStock } & 13.4 \\ \text { LCBond } & 4.8 \\ \text { LGBond } & 4.2 \\ \text { TBill } & 3.6 \\ \text { RealEst } & 0.0\end{array}$

Parameters STD(i) standard deviations of individual assets (\%)

\begin{tabular}{|lr} 
/ LaStock & 20.5 \\
SmStock & 26.7 \\
LCBond & 7.5 \\
LGBond & 6.7 \\
TBill & 3.3 \\
RealEst & $0.0 \quad /$;
\end{tabular}

Table col(i,j) correlations between assets

$\begin{array}{lcccccc} & \text { LaStock } & \text { SmStock } & \text { LCBond } & \text { LGBond } & \text { TBill } & \text { RealEst } \\ \text { LaStock } & 1.00 & 0.82 & 0.19 & 0.11 & -0.07 & -0.06 \\ \text { SmStock } & 0.82 & 1.00 & 0.08 & -0.01 & -0.08 & 0.06 \\ \text { LCBond } & 0.19 & 0.08 & 1.00 & 0.93 & 0.19 & -0.08 \\ \text { LGBond } & 0.11 & -0.01 & 0.93 & 1.00 & 0.22 & -0.09 \\ \text { TBill } & -0.07 & -0.08 & 0.19 & 0.22 & 1.00 & 0.38 \\ \text { RealEst } & -0.06 & 0.06 & -0.08 & -0.09 & 0.38 & 1.00\end{array}$


$\begin{array}{lll}\text { Variables } & \mathbf{x}(\mathrm{i}) & \text { fraction of portfolio invested in asset } \mathrm{i} \\ \text { variance } & \begin{array}{l}\text { variance of portfolio } \\ \text { standev }\end{array} & \text { standard deviation of portfolio }\end{array}$

Positive Variable $\mathrm{x}$;

Equations fsum fractions must add to 1.0

dmean definition of expected return on portfolio

fvariance definition of portfolio variance;

fsum..

$\operatorname{sum}(i, x(i))$

$=\mathrm{e}=1.0$;

dmean.

$\operatorname{sum}(i, \operatorname{return}(i) * x(i))$

$=\mathrm{e}=$ target ;

fvariance..

$\operatorname{sum}(i, x(i) * \operatorname{sum}(j, \operatorname{col}(i, j) * \operatorname{STD}(i) * \operatorname{STD}(j) * x(j)))=e=$ variance;

Model portfolio / all /;

Solve portfolio using nlp minimizing variance;

standev.l = sqrt(variance.l) ;

Display target, variance.l, standev.l, x.l;

For $(k=1$ to 5 ,

$$
\text { target }=\text { target }+0.5 \text {; }
$$

* If used for generating aggressive portfolio list, target $=$ target - step

* In this example, the length of step is set at 0.5

Solve portfolio using nlp minimizing variance;

standev.1 = sqrt(variance.l) ;

Display target, variance.l, standev.l, x.l;

); 


\section{Appendix 6.3}

\section{GAMS Program for Finding the Maximum Return Portfolio in terms of the Liquidity Objective}

\$OnText

This GAMS program is written to find the maximum return portfolio along the efficient frontier in terms of the liquidity objective (LD). This portfolio is considered the most aggressive. The underlying quadratic programming model is Model 2.1.

\section{SOffText}

Set i assets /LaStock, SmStock,LCBond, LGBond,TBill, RealEst/; alias (i,j);

Scalar $k$ index for loop;

Scalar TargetSTD target STD on portfolio (\%) \ $^{\prime}$ /23.7/;

Parameters return(i) expected liquidity-adjusted returns of individual assets (\%)

$\begin{array}{lr}\text { LaStock } & 11.9 \\ \text { SmStock } & 13.4 \\ \text { LCBond } & 4.8 \\ \text { LGBond } & 4.2 \\ \text { TBill } & 3.6 \\ \text { RealEst } & 0.0 \quad / \text {; }\end{array}$

Parameters STD(i) standard deviations of individual assets (\%)

$\begin{array}{lr}\text { / LaStock } & 20.5 \\ \text { SmStock } & 26.7 \\ \text { LCBond } & 7.5 \\ \text { LGBond } & 6.7 \\ \text { TBill } & 3.3 \\ \text { RealEst } & 0.0 \quad / \text {; }\end{array}$

Table col( $i, j)$ correlations between assets

$\begin{array}{lcccccc} & \text { LaStock } & \text { SmStock } & \text { LCBond } & \text { LGBond } & \text { TBill } & \text { RealEst } \\ \text { LaStock } & 1.00 & 0.82 & 0.19 & 0.11 & -0.07 & -0.06 \\ \text { SmStock } & 0.82 & 1.00 & 0.08 & -0.01 & -0.08 & 0.06 \\ \text { LCBond } & 0.19 & 0.08 & 1.00 & 0.93 & 0.19 & -0.08 \\ \text { LGBond } & 0.11 & -0.01 & 0.93 & 1.00 & 0.22 & -0.09 \\ \text { TBill } & -0.07 & -0.08 & 0.19 & 0.22 & 1.00 & 0.38 \\ \text { RealEst } & -0.06 & 0.06 & -0.08 & -0.09 & 0.38 & 1.00\end{array}$


Variables $\quad x(i) \quad$ fraction of portfolio invested in asset $i$

Positive Variable x;

ExReturn expected return on portfolio

Equations

fsum fractions must add to 1.0

dmean definition of expected return on portfolio

FSTD definition of standard deviation of portfolio;

fsum.. $\quad \operatorname{sum}(\mathrm{i}, \mathrm{x}(\mathrm{i})) \quad=\mathrm{e}=1.0 \quad$;

dmean.. $\quad \operatorname{sum}(i$, return $(i) * x(i)) \quad=e=$ ExReturn;

fSTD.; $\quad \operatorname{sqrt}(\operatorname{sum}(i, x(i) * \operatorname{sum}(j, \operatorname{col}(i, j) * \operatorname{STD}(i) * \operatorname{STD}(j) * x(j))))=e=$ TargetSTD;

Model portfolio / all /;

Solve portfolio using nlp maximizing ExReturn;

Display ExReturn.1, targetSTD, x.1 ;

* Now change the value of targetSTD

targetSTD $=$ targetSTD $+0.2 ;$

Scalars R1;

Scalars R2;

R1 = ExReturn.1;

Model p1 /fsum, dmean, fSTD/;

Solve p1 using nlp maximizing ExReturn;

R2 = ExReturn.l;

$k=1$;

For $(k=1$ to 20 ,

If $(R 1<R 2$,

targetSTD $=$ targetSTD +0.2 ;

$\mathrm{R} 1=\mathrm{R} 2$;

Solve p1 using nlp maximizing ExReturn;

R2 = ExReturn.1;

Else

targetSTD $=$ targetSTD -0.2 ;

Solve pl using nlp maximizing ExReturn;

Display ExReturn.l, targetSTD, x.1 ;

$\operatorname{targetSTD}=\operatorname{targetSTD}+0.2$;

); 


\section{Appendix 6.4}

\section{GAMS Program for Obtaining the Local Optimal Portfolio in terms of the Liquidity Objective}

\section{\$OnText}

This GAMS program is written to solve the quadratic programming model (Model 4.2) whose objective function is to maximize an investor's utility in terms of the liquidity objective (LD). The outputs are the characteristics of local optimal portfolio, including the expected return, standard deviation, percentage of portfolio invested in each asset class, as well as the values of the investor's risk tolerance and utility.

\section{sOffText}

Set i assets /LaStock, SmStock,LCBond, LGBond,TBill, RealEst/; alias (i,j);

Set $m$ reference portfolio $A / \operatorname{ReA}, \operatorname{Var} A /$;

Set 1 reference portfolio $\mathrm{C} / \mathrm{ReC}, \operatorname{VarC} /$;

Scalar $t$ the investor's risk tolerance;

Scalar $k$ the relative degree of preference between portfolios A and C / 0.630/;

* For aggressive action, $\mathrm{k}=0.475$

* For normal action, $\mathrm{k}=0.630$

* For conservative action, $\mathrm{k}=2.759$

Parameters refA(m) expected return and variance of reference portfolio $A$

/ ReA $\quad 3.00$

$\operatorname{VarA} 5.157 /$;

Parameters refC(l) expected return and variance of reference portfolio $\mathrm{C}$

$$
\text { / ReC } \quad 10.00
$$

$\operatorname{VarC} \quad 233.60 /$;

$\mathrm{t}=\left(\operatorname{refA}\left({ }^{\prime} \operatorname{Var} \mathrm{A}^{\prime}\right)-\mathrm{k} * \operatorname{refC}\left({ }^{\prime} \operatorname{Var} C^{\prime}\right)\right) /\left(\operatorname{refA}\left({ }^{\prime} \operatorname{ReA}{ }^{\prime}\right)-\mathrm{k} * \operatorname{refC}\left({ }^{\prime} \operatorname{ReC} C^{\prime}\right)\right)$

Parameters return(i) expected liquidity-adjusted returns of individual assets.(\%)

$\begin{array}{llr}\text { / LaStock } & 11.9 \\ \text { SmStock } & 13.4 \\ \text { LCBond } & 4.8 \\ \text { LGBond } & 4.2 \\ \text { TBill } & 3.6 \\ \text { RealEst } & 0.0 \text { /; }\end{array}$


Parameters STD(i) standard deviations of individual assets (\%)

$\begin{array}{lr}\text { LaStock } & 20.5 \\ \text { SmStock } & 26.7 \\ \text { LCBond } & 7.5 \\ \text { LGBond } & 6.7 \\ \text { TBill } & 3.3 \\ \text { RealEst } & 0.0 \%\end{array}$

Table col(i,j) correlations between assets

$\begin{array}{lcccccc} & \text { LaStock } & \text { SmStock } & \text { LCBond } & \text { LGBond } & \text { TBill } & \text { RealEst } \\ \text { LaStock } & 1.00 & 0.82 & 0.19 & 0.11 & -0.07 & -0.06 \\ \text { SmStock } & 0.82 & 1.00 & 0.08 & -0.01 & -0.08 & 0.06 \\ \text { LCBond } & 0.19 & 0.08 & 1.00 & 0.93 & 0.19 & -0.08 \\ \text { LGBond } & 0.11 & -0.01 & 0.93 & 1.00 & 0.22 & -0.09 \\ \text { TBill } & -0.07 & -0.08 & 0.19 & 0.22 & 1.00 & 0.38 \\ \text { RealEst } & -0.06 & 0.06 & -0.08 & -0.09 & 0.38 & 1.00\end{array}$

Variables $\quad x(i) \quad$ fraction of portfolio invested in asset $i$

returnEx expected return of portfolio

variance definition of portfolio variance

standev standard deviation of portfolio

utility definition of utility;

Positive Variable $\mathrm{x}$;

Equations

fsum fractions must add to 1.0

freturnEx definition of portfolio return

fvariance definition of portfolio variance

dutil definition of utility;

fsum.. $\quad \operatorname{sum}(i, x(i))$

freturnEx.. $\operatorname{sum}(i, \operatorname{return}(i) * x(i))$

fvariance.. $\operatorname{sum}(i, x(i) * \operatorname{sum}(j, \operatorname{col}(i, j) * \operatorname{STD}(i) * \operatorname{STD}(i) * x(j)))$

dutil.. returnEx - variance/t $=\mathrm{e}=1.0$;

$=\mathrm{e}=$ returnEx;

$=\mathrm{e}$ variance;

$=e=$ utility ;

Model portfolio / all/;

Solve portfolio using nlp maximizing utility;

standev.l = sqrt(variance.l) ;

Display $\mathrm{t}$, utility.1, returnEx.1, standev.1, x.1 ; 


\section{$\therefore$ Appendix 6.5 .}

\section{GAMS Program for Finding Performance of the Complete Portfolio in terms of the Liquidity Objective}

\section{SOnText}

This GAMS program is written to obtain the performance information (expected return and standard deviation) of the complete portfolio in terms of the liquidity objective (LD).

SOffText

Set i assets /LaStock, SmStock,LCBond, LGBond,TBill, RealEst/; alias (i,j)

Scalar ReturnC Excepted return of complete portfolio for LD objective VarC Variance of complete portfolio for $L D$ objective

StdC Standard deviation complete portfolio for LD objective;

Parameters x(i) fraction of assets making up the complete portfolio

\begin{tabular}{lll}
\hline LaStock & 0.282 \\
SmStock & 0.164 \\
LCBond & 0.177 \\
LGBond & 0.000 \\
TBill & 0.040 \\
RealEst & $0.337 \quad /$;
\end{tabular}

*These data are for normal action;

$*$ For aggressive action, $\mathrm{LaStock}=0.286$, SmStock $=0.266$; $\mathrm{LCB}$ ond $=0.148$,

* LGBond $=0.000, \mathrm{TBill}=0.009$, RealEst $=0.291$.

$*$ For conservative action, LaStock $=0.232$, SmStock $=0.081, \mathrm{LCB}$ ond $=0.283$,

* LGBond $=0.001$, TBill $=0.075$, RealEst $=0.328$.

Parameters return(i) expected liquidity-adjusted returns of individual assets (\%)

$\begin{array}{llr}\text { LaStock } & 11.9 \\ \text { SmStock } & 13.4 \\ \text { LCBond } & 4.8 \\ \text { LGBond } & 4.2 \\ \text { TBill } & 3.6 \\ \text { RealEst } & 0.0 / \text {; }\end{array}$


Parameters STD(i) standard deviations of individual assets (\%)

$\begin{array}{llr}\text { / LaStock } & 20.5 \\ \text { SmStock } & 26.7 \\ \text { LCBond } & 7.5 \\ \text { LGBond } & 6.7 \\ \text { TBill } & 3.3 \\ \text { RealEst } & 0.0 \quad / \text {; }\end{array}$

Table col(i,j) correlations between assets

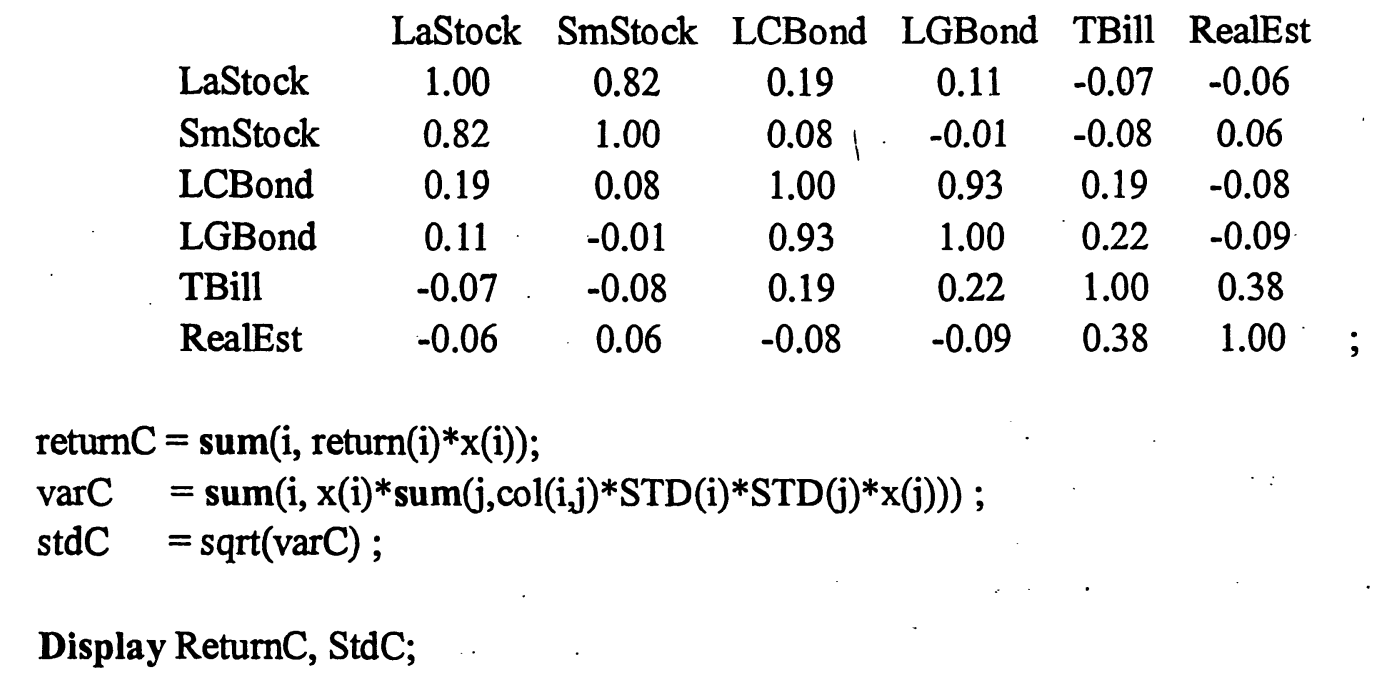




\section{Appendix 6.6}

\section{GAMS Program for Obtaining the Optimal Portfolio for a Given Target Return in terms of the Liquidity Objective}

\section{SOnText}

This GAMS program is written to solve the quadratic programming model (Model 4.1) whose objective function is to minimize a portfolio's variance in terms of the liquidity objective (LD). The input data are the specified expected target returns. The outputs are the characteristics of optimal portfolio, including the expected return, standard deviation, percentage of portfolio invested in each asset class.

\$OffText

Set $\quad i$ assets /LaStock, SmStock,LCBond, LGBond,TBill, RealEst/; alias (i,j)

Scalar target target mean liquidity-adjusted return of portfolio (\%) / 10/;

Parameters return(i) expected liquidity-adjusted returns of individual assets (\%)

$\begin{array}{llr}\text { / LaStock } & 11.9 \\ \text { SmStock } & 13.4 \\ \text { LCBond } & 4.8 \\ \text { LGBond } & 4.2 \\ \text { TBill } & 3.6 \\ \text { RealEst } & 0.0 \quad / \text {; }\end{array}$

Parameters STD(i) standard deviations of individual assets (\%)

$\begin{array}{llr}\text { / LaStock } & 20.5 \\ \text { SmStock } & 26.7 \\ \text { LCBond } & 7.5 \\ \text { LGBond } & 6.7 \\ \text { TBill } & 3.3 \\ \text { RealEst } & 0.0 \quad / \text {; }\end{array}$

Table col( $i, j)$ correlations between assets

$\begin{array}{lcccccc} & \text { LaStock } & \text { SmStock } & \text { LCBond } & \text { LGBond } & \text { TBill } & \text { RealEst } \\ \text { LaStock } & 1.00 & 0.82 & 0.19 & 0.11 & -0.07 & -0.06 \\ \text { SmStock } & 0.82 & 1.00 & 0.08 & -0.01 & -0.08 & 0.06 \\ \text { LCBond } & 0.19 & 0.08 & 1.00 & 0.93 & 0.19 & -0.08 \\ \text { LGBond } & 0.11 & -0.01 & 0.93 & 1.00 & 0.22 & -0.09 \\ \text { TBill } & -0.07 & -0.08 & 0.19 & 0.22 & 1.00 & 0.38 \\ \text { RealEst } & -0.06 & 0.06 & -0.08 & -0.09 & 0.38 & 1.00\end{array}$


Variables $\quad x(i) \quad$ fraction of portfolio invested in asset $i$

variance variance of portfolio

standev standard deviation of portfolio

\section{Positive Variable x;}

Equations fsum fractions must add to 1.0

dmean definition of expected return on portfolio

fvariance definition of portfolio variance;

fsum.. $\quad \operatorname{sum}(i, x(i))$

dmean.. $\quad \operatorname{sum}(i$, return $(i) * x(i))$

$=\mathrm{e}=1.0 ;$

fvariance..

$\operatorname{sum}(i, x(i) * \operatorname{sum}(j, \operatorname{col}(i, j) * \operatorname{STD}(i) * \operatorname{STD}(j) * x(j)))$

$=\mathrm{e}=$ target;

$=\mathrm{e}=$ variance;

Model portfolio / all /;

Solve portfolio using nlp minimizing variance;

standev.1 = sqrt(variance.l) ;

Display target, variance.l, standev.l, x.l; 


\section{References}

AIMR (2003). "Code of ethics and standards of professional conduct". Association for Investment Management and Research, Charlottesville, Virginia, U.S.A., http://www.aimr.com/pdf/standards/english_code.pdf, accessed on July 20, 2003.

Binhammer, H.H. and Sephton, P. (1998). 'Money, Banking and the Canadian Financial System, $7^{\text {th }}$ Edition. Internatonal Thomson Publishing, Scarborough, Ontario, Canada.

Bodie, Z., Kane, A., Marcus, A.J., Perrakis, S. and Ryan, P.J. (1997). Investments, $2^{\text {nd }}$ Canadian Edition. McGraw-Hill Ryerson Limited, Whitby, Ontario, Canada.

Bolster, P.J., Janjigian, V., and Trahan E.A. (1995). "Determining investor suitability using the Analytic Hierarchy Process". Financial Analysts Journal, Vol. 51, No. 4, pp. 63-75.

Bolster, P.J. and Warrick, S. (2000). "Suitability and optimality in the asset allocation process". Working paper, Northeastern University, Boston, Massachusetts, U.S.A., http://www.northinfo.com/papers/pdf/19990901_ahp.pdf, accessed on July 5, 2003.

$\mathrm{CCH}$ (2003). Canadian Income Tax Act and with Regulations, $75^{\text {th }}$ Edition. CCH Canadian Limited, Toronto, Ontario, Canada.

Chriss, N.A. and Fanelli, P.R. (1998). "Portfolio risk measurement". In Krause, R.P. (ed.), Global Equity and Derivative Markets: Risk, pp. 52-59. Morgan Stanley Dean Witter, New York, U.S.A.

Ciochetti, B.A., Fisher, J.D., and Gao, B. (2003). "A structured model approach to estimating return and volatility for commercial real estate". The Real Estate Research Institute, Hartford, Connecticut, U.S.A., http://www.reri.org/research/pdf/wp116.pdf, accessed on July 4, 2003.

Cohen, J.B., Zinbarg, E.D., and Zeikel, A. (1987). Investment Analysis and Portfolio Management, $5^{\text {th }}$ Edition. Irwin, Homewood, Mlinois, U.S.A.

Droms, G.W. and Strauss, S.N. (2003). "Assessing risk tolerance for asset allocation". Journal of Financial Planning, Vol. 16, No. 3, pp. 72-77.

Elton, E.J. and Gruber, M.J. (1991). Modern Portfolio Theory and Investment Analysis, $4^{\text {th }}$ Edition. Wiley, New York, U.S.A.

Elton, E.J. and Gruber, M.J. (1997). "Modern portfolio theory, 1950 to date". Journal of Banking \& Finance, Vol. 21, pp. 1743-1759. 
Fama, E.F. (1995). "Random walks in stock market prices". Financial Analysts Journal, Vol. 51, No. 1, pp. 75-80.

GAMS Development Corporation, Washington, D.C., U.S.A., www.gams.com

Hamza, F. and Janssen, J. (1998). "The mean-semivariances approach to realistic portfolio optmization subject to transaction costs". Applied Stochastic Models and Data Analysis, 14, pp. 275-283.

Hobbs, B.F. and Meier, P. (2000). Energy Decisions and the Environment: $A$ Guide to the Use of Multi-criteria Methods. Kluwer Academic Publishers, Boston, Massachusetts, U.S.A.

Information Please (2003). "What's the payoff? Historical returns of the asset classes". Pearson Education, Upper Saddle River, New Jersey, U.S.A., http://www.infoplease.com/finance/education/wall/wall_inv_hist.html, accessed on August 29, 2003.

Ishizaka, A. and Lusti, M. (2003). "An expert module to improve the consistency of AHP matrices". University of Basel, Switzerland, http://www.wwz.unibas.ch/wi/members/Ishizaka/IFORS2002.pdf, accessed on July 2, 2003.

Jones, C.P. (1998). Investments: Analysis and Management, $6^{\text {th }}$ Edition. Wiley, New York, U.S.A.

Khaksari, S., Kamath, R. and Grieves, R. (1989). "A new approach to determining optimum portfolio mix." Journal of Portfolio Management, Vol. 15, No. 3, pp. 43-49.

Konno, H. and Yamazaki, H. (1991). "Mean-absolute deviation portfolio optimization model and its applications to Tokyo stock market". Management Science, 37, pp. 519-531.

Maginn, J.L. and Tuttle, D.L. (ed.) (1990). Managing Investment Portfolios: A Dynamic Process. Association for Investment Management and Research, Charlottesville, Virginia, U.S.A.

Markowitz, H. (1952). "Portfolio selection". Journal of Finance, Vol. 7, pp. 77-91.

Michaud, R.O. (1998). Efficient Asset Management: A Practical Guide to Stock Portfolio Optimization and Asset Allocation. Harvard Business School Press, Boston, Massachusetts, U.S.A.

Muralidharan, C, Anantharaman, N. and Deshmukh, S.G (2002). "A multi-criteria group decision making model for the supplier rating". Journal of Supply Chain Management, Vol. 
38 , No. 4 , pp. 22-33.

New York Institute of Finance. "Assessing your risk tolerance", http://www.ici.org/i4s/i4s_risk_tolerance.pdf, accessed on July 14, 2003.

Peters, M.L and Zelewski, S. (2003). "A heuristic algorithm to improve the consistency of judgments in the Analytical Hierarchy Process (AHP) ". University of Essen, Germany, http://www.pim.uni-essen.de/publikationen/peters/bericht18.pdf, accessed on July 2, 2003.

Polsky L. (1998). "Towards a more dynamic and realistic approach to risk measurement". In Krause, R.P. (ed.), Global Equity and Derivative Markets: Risk, p. 15. Morgan Stanley Dean Witter, New York, U.S.A.

RBC Investments (2003). Action Directions: A quarterly publication for Action Direct clients, Summer. RBC Investments, Toronto, Ontario, Canada.

Ross, S.A. (1976). "The arbitrage theory of capital asset pricing". Journal of Economic Theory, 13, pp. 341-360.

Royal Swedish Academy of Sciences. "The Sveriges Riksbank (Bank of Sweden) Prize in Economic Sciences in memory of Alfred Nobel for 1990", Press Release, http://www.nobel.se/economics/laureates/1990/press.html, accessed on July 12, 2003.

Saaty, T.L. (1980). The Analytic Hierarchy Process: Planning, Setting Priority, Resource Allocation. McGraw-Hill, New York, U.S.A.

Saaty, T.L. (1982). Decision Making for Leaders: the Analytical Hierarchy Process for Decision in a Complex World. Wadsworth, California, U.S.A.

Saaty, T. L. (1994). "How to make a decision: the Analytical Hierarchy Process". Interfaces, Vol. 24, No. 6, pp. 19-43.

Saraoglu, H. and Detzler, M.L. (2002). "A sensible mutual fund selection model". Financial Analysts Journal, Vol. 58, No. 3, pp. 60-72.

Sharpe, W.F. (1963). "A simplified model of portfolio analysis". Management Science, Vol. 9, No.1, pp. 277-293.

Sharpe, W.F. (1964). "Capital asset prices: a theory of market equilibrium under conditions of risk". Journal of Finance, Vol.19, September, pp. 425-442.

Sharpe, W.F. (1967). "A linear programming algorithm for mutual fund portfolio selection". Management Science, 13, pp. 499-510. 
Sharpe, W.F. (1971). "A linear programming approximation for the general portfolio analysis problem". Journal of Financial Quantitative Analysis, December, pp. 1263-1275.

Sharpe, W.F. (1987). "Integrated asset allocation". Financial Analysts Journal, Vol. 43, No.5, pp. 25-32.

Speranza, M.G (1996). "A heuristic algorithm for a portfolio optimization model applied to the Milan stock market". Computers \& Operations Research, Vol. 23, No.5, pp. 433-441.

Stone, B.K. (1973). "A linear programming formulation of the general portfolio selection problem". Journal of Financial Quantitative Analysis, September, pp. 621-636.

Vanguard Group. "Investor questionnaire". http://www.vanguard.com/pdf/wiquestion.pdf, accessed on July 14, 2003.

$\mathrm{Xu}, Z$. and Wei, C. (1999). "A consistency improving method in the Analytic Hierarchy Process." European Journal of Operational Research, Vol. 116, No. 2, pp. 443-449. 